\title{
DIMENSION DEPENDENCE OF FACTORIZATION PROBLEMS: BI-PARAMETER HARDY SPACES
}

\author{
RICHARD LECHNER
}

\begin{abstract}
Given $1 \leq p, q<\infty$ and $n \in \mathbb{N}_{0}$, let $H_{n}^{p}\left(H_{n}^{q}\right)$ denote the canonical finite-dimensional bi-parameter dyadic Hardy space. Let $\left(V_{n}: n \in \mathbb{N}_{0}\right)$ denote either $\left(H_{n}^{p}\left(H_{n}^{q}\right): n \in \mathbb{N}_{0}\right)$ or $\left(\left(H_{n}^{p}\left(H_{n}^{q}\right)\right)^{*}: n \in \mathbb{N}_{0}\right)$. We show that the identity operator on $V_{n}$ factors through any operator $T: V_{N} \rightarrow V_{N}$ which has large diagonal with respect to the Haar system, where $N$ depends linearly on $n$.
\end{abstract}

\section{INTRODUCTION}

For each $n \in \mathbb{N}$, suppose that $V_{n}$ has a normalized 1-unconditional basis $e_{j}, 1 \leq$ $j \leq n$, and let $e_{j}^{*} \in V_{n}^{*}, 1 \leq j \leq n$ denote the associated coordinate functionals. This work is concerned with the following question:

Question 1.1. Given $n \in \mathbb{N}$ and $\delta, \Gamma, \eta>0$, what is the smallest integer $N=$ $N(n, \delta, \Gamma, \eta)$, such that for any operator $T: V_{N} \rightarrow V_{N}$ satisfying

$$
\|T\| \leq \Gamma \quad \text { and } \quad\left|\left\langle e_{j}^{*}, T e_{j}\right\rangle\right| \geq \delta, \quad 1 \leq j \leq N,
$$

there are there operators $E: V_{n} \rightarrow V_{N}$ and $F: V_{N} \rightarrow V_{n}$, such that the diagram

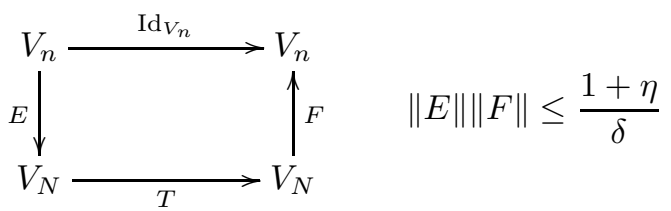

is commutative?

In numerous Banach spaces, there exist quantitative estimates for $N$ (see e.g. 2, 3, 10, 1, 14, 13, 12, 9, 6, 7, 8,). To illustrate: the estimate for the relationship between $N$ and $n$ is

$\triangleright$ linear for $V_{n}=\ell_{n}^{p}, 1 \leq p \leq \infty$ (see e.g. [3]);

$\triangleright$ polynomial for the one-parameter dyadic Hardy spaces $H_{n}^{p}, 1 \leq p<\infty$ (see Section 2 for the definition of $H_{n}^{p}$ ) and their duals (see [8]).

However, in many other Banach spaces the best known estimates for $N$ are often super-exponential. To illustrate, put $d_{n}=2^{n+1}-1, n \in \mathbb{N}_{0}$, and let $H_{n}^{p}\left(H_{n}^{q}\right), 1 \leq$ $p, q<\infty$ denote the bi-parameter mixed norm dyadic Hardy space with dimension $d_{n}^{2}$ (see Section 2 for the definition of $H_{n}^{p}\left(H_{n}^{q}\right)$ ). The best known estimate for $V_{d_{n}^{2}}=H_{n}^{p}\left(H_{n}^{q}\right)$ and $V_{d_{n}^{2}}=\left(H_{n}^{p}\left(H_{n}^{q}\right)\right)^{*}, 1 \leq p, q<\infty$ is a nested exponential (see [6]), e.g. of the form

$$
N \leq 2^{8^{n} 2^{8^{n-1} 2^{8^{n-2} 2^{8^{n-3}}} \cdot \cdots}}
$$

Date: April 26, 2022.

2010 Mathematics Subject Classification. 46B07,30H10,46B25,60G46.

Key words and phrases. Factorization, local theory, almost-diagonalization, classical Banach spaces, Hardy spaces, BMO.

Supported by the Austrian Science Foundation (FWF) Pr.Nr. P28352. 
In this work, we use the new probabilistic method introduced in [8], to improve the super-exponential estimate (1.3) to the linear estimate

$$
N \leq c n, \quad \text { where } c=c(\delta, \Gamma, \eta)>0 .
$$

\section{Notation}

Let $\mathcal{D}$ denote the dyadic intervals contained in the unit interval $[0,1)$, i.e.

$$
\mathcal{D}=\left\{\left[(k-1) 2^{-n}, k 2^{-n}\right): n \in \mathbb{N}_{0}, 1 \leq k \leq 2^{n}\right\} .
$$

Let $|\cdot|$ denote the Lebesgue measure. For any $N \in \mathbb{N}_{0}$, we put

$$
\mathcal{D}_{N}=\left\{I \in \mathcal{D}:|I|=2^{-N}\right\} \quad \text { and } \quad \mathcal{D}_{\leq N}=\bigcup_{n=0}^{N} \mathcal{D}_{n} .
$$

Given $n \in \mathbb{N}_{0}$ and a dyadic interval $I \in \mathcal{D}_{n}$, we define $I^{-}, I^{+} \in \mathcal{D}_{n+1}$ by

$$
I^{+} \cup I^{-}=I \quad \text { and } \quad \inf I^{+}<\inf I^{-} .
$$

For any two collections $\mathcal{A}, \mathcal{B} \subset \mathcal{D}$, we introduce the following notation:

$$
\mathcal{A} \otimes \mathcal{B}=\{I \times J: I \in \mathcal{A}, J \in \mathcal{B}\} .
$$

The $L^{\infty}$-normalized Haar system $h_{I}, I \in \mathcal{D}$ is given by

$$
h_{I}=\chi_{I^{+}}-\chi_{I^{-}}, \quad I \in \mathcal{D},
$$

where $\chi_{A}$ denotes the characteristic function of the set $A \subset[0,1)$. Given $1 \leq p<\infty$, the one-parameter dyadic Hardy space $H^{p}$ is the completion of

$$
\operatorname{span}\left\{h_{I}: I \in \mathcal{D}\right\}
$$

under the square function norm

$$
\|f\|_{H^{p}}=\left(\int_{0}^{1}\left(\sum_{I}\left|a_{I}\right|^{2} h_{I}^{2}(x)\right)^{p / 2} \mathrm{~d} x\right)^{1 / p},
$$

where $f=\sum_{I} a_{I} h_{I}$. For all $n \in \mathbb{N}_{0}$, we define the finite-dimensional subspaces $H_{n}^{p}$ of $H^{p}$ by

$$
H_{n}^{p}=\operatorname{span}\left\{h_{I}: I \in \mathcal{D}_{\leq n}\right\} .
$$

The bi-parameter $L^{\infty}$-normalized Haar system $h_{I \times J}, I, J \in \mathcal{D}$ is given by

$$
h_{I \times J}=h_{I} \otimes h_{J}, \quad I, J \in \mathcal{D},
$$

where the tensor product of two functions $f, g:[0,1) \rightarrow \mathbb{R}$ is defined by

$$
(f \otimes g)(x, y)=f(x) g(x), \quad x, y \in[0,1) .
$$

For $1 \leq p, q<\infty$, the bi-parameter dyadic Hardy space $H^{p}\left(H^{q}\right)$ is the completion of

$$
\operatorname{span}\left\{h_{R}: R \in \mathcal{D} \otimes \mathcal{D}\right\}
$$

under the square function norm

$$
\|f\|_{H^{p}\left(H^{q}\right)}=\left(\int_{0}^{1}\left(\int_{0}^{1}\left(\sum_{R \in \mathcal{D} \otimes \mathcal{D}}\left|a_{R}\right|^{2} h_{R}^{2}(x, y)\right)^{q / 2} \mathrm{~d} y\right)^{p / q} \mathrm{~d} x\right)^{1 / p},
$$

where $f=\sum_{R \in \mathcal{D} \otimes \mathcal{D}} a_{R} h_{R}$. For each $n \in \mathbb{N}_{0}$, we define the finite-dimensional subspace $H_{n}^{p}\left(H_{n}^{q}\right)$ of $H^{p}\left(H^{q}\right)$ by

$$
H_{n}^{p}\left(H_{n}^{q}\right)=\operatorname{span}\left\{h_{R}: R \in \mathcal{D}_{\leq n} \otimes \mathcal{D}_{\leq n}\right\} .
$$




\section{MAIN RESUlT}

Recall that we put $d_{n}=2^{n+1}-1, n \in \mathbb{N}_{0}$, and let $1 \leq p, q<\infty$. We give a quantitative estimate for the $N$ appearing in Question 1.1 for the spaces $V_{d_{n}}=$ $H_{n}^{p}\left(H_{n}^{q}\right)$ and $V_{d_{n}}=\left(H_{n}^{p}\left(H_{n}^{q}\right)\right)^{*}$. In particular, the relation between $N$ and $n$ is linear.

Theorem 3.1. Let $1 \leq p, q<\infty$, and let $\left(V_{k}: k \in \mathbb{N}_{0}\right)$ denote either

$$
\left(H_{k}^{p}\left(H_{k}^{q}\right): k \in \mathbb{N}_{0}\right) \quad \text { or } \quad\left(\left(H_{k}^{p}\left(H_{k}^{q}\right)\right)^{*}: k \in \mathbb{N}_{0}\right) .
$$

Let $n \in \mathbb{N}_{0}$ and $\delta, \Gamma, \eta>0$. Define the integer $N=N(n, \delta, \Gamma, \eta)$ by the formula

$$
N=41(n+3)+\left\lfloor 4 \log _{2}(\Gamma / \delta)+4 \log _{2}\left(1+\eta^{-1}\right)\right\rfloor .
$$

Then for any operator $T: V_{N} \rightarrow V_{N}$ satisfying

$$
\|T\| \leq \Gamma \quad \text { and } \quad\left|\left\langle T h_{Q}, h_{Q}\right\rangle\right| \geq \delta|Q|, \quad Q \in \mathcal{D}_{\leq N} \otimes \mathcal{D}_{\leq N},
$$

there exist bounded linear operators $E: V_{n} \rightarrow V_{N}$ and $F: V_{N} \rightarrow V_{n}$, such that the diagram

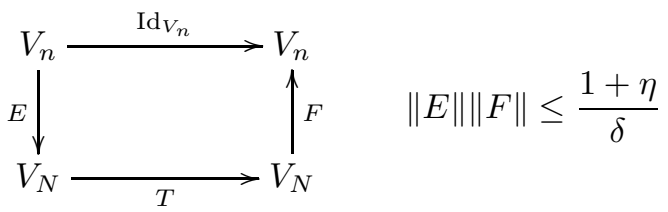

is commutative.

Note that the linear relation between $N$ and $n$ amounts to a polynomial relation between the dimensions of the respective spaces; i.e. $\operatorname{dim} V_{N}$ is a polynomial in $\operatorname{dim} V_{n}$.

Formula (3.2) is the main focus of this work. Specifically, we improve the previously best known estimate for the relation between $N$ and $n$ in $H_{N}^{p}\left(H_{N}^{q}\right)$ and $\left(H_{N}^{p}\left(H_{N}^{q}\right)\right)^{*}, 1 \leq p, q<\infty$ (see [6]), from super-exponential to linear (which means from (1.3) to (1.4)). The super-exponential growth in [6] is caused by the use of combinatorics. The same is true even in one-parameter spaces (see e.g. [10, 12, 9, 17]).

Recently, using a probabilistic approach (see [8]), linear estimates for $N$ in $n$ were obtained in the context of one-parameter spaces. In this work, we extend this probabilistic method to the bi-parameter spaces $H_{N}^{p}\left(H_{N}^{q}\right)$ and $\left(H_{N}^{p}\left(H_{N}^{q}\right)\right)^{*}$, $1 \leq p, q<\infty$, and thereby obtain the formula (3.1).

\section{Tensor Products, EMbeddings And Projections In MIXED NORM SPACES}

This section consists of two major parts: The first part connects Jones' compatibility condition (J) to Capon's local product condition (P1)-( we show that every operator on a bi-parameter Hardy space is almost-diagonalized by a properly constructed randomized block basis. Both parts are vital components in the proof of our main result Theorem 3.1 .

4.1. Jones' compatibility condition and Capon's local product condition. Given $z_{I} \subset \mathcal{D}, I \in \mathcal{D}$, we put $Z_{I}=\bigcup z_{I}$. We say that the collections $z_{I}, I \in \mathcal{D}$ satisfy Jones' compatibility condition (J) (see [4]; see also [11]) with constant $\kappa \geq 1$, if the following four conditions are satisfied:

(J1) For each $I \in \mathcal{D}$, the collection $z_{I}$ consists of finitely many pairwise disjoint dyadic intervals; moreover, $z_{I} \cap z_{I^{\prime}}=\emptyset$, whenever $I, I^{\prime} \in \mathcal{D}, I \neq I^{\prime}$.

(J2) For every $I \in \mathcal{D}$, we have that $Z_{I^{-}} \cup Z_{I^{+}} \subset Z_{I}$ and $Z_{I^{-}} \cap Z_{I^{+}}=\emptyset$.

(J3) $\kappa^{-1}|I| \leq\left|Z_{I}\right| \leq \kappa|I|$, for all $I \in \mathcal{D}$.

(J4) For all $I_{0}, I \in \mathcal{D}$ with $I_{0} \subset I$ and $K \in Z_{I}$, we have $\frac{\left|K \cap Z_{I_{0}}\right|}{|K|} \geq \kappa^{-1} \frac{\left|Z_{I_{0}}\right|}{\left|Z_{I}\right|}$. 
Jones' compatibility condition (J) is crucial to construct block bases of the Haar system onto which the natural projection is bounded in $H^{1}$; especially (J4). Lemma 4.1 below asserts that the tensor product of collections satisfying Jones' compatibility condition (J) satisfies Capon's local product condition (P1)-(P4) (see [5]). Capon's local product condition is used to construct block bases of the bi-parameter Haar system onto which the natural projection onto that block basis

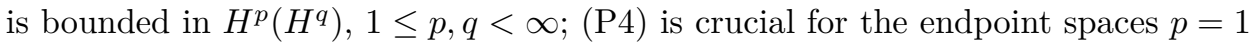
or $q=1$.

Lemma 4.1. Let $X_{I} \subset \mathcal{D}, I \in \mathcal{D}$ and $y_{J} \subset \mathcal{D}, J \in \mathcal{D}$ both satisfy condition (J) with constant $\kappa \geq 1$. Define

$$
\mathcal{B}_{I \times J}=x_{I} \otimes y_{J}=\left\{K \times L: K \in x_{I}, L \in y_{J}\right\}, \quad I, J \in \mathcal{D},
$$

and put

$$
X_{I}=\bigcup x_{I}, \quad I \in \mathcal{D} \quad \text { as well as } \quad Y_{J}=\bigcup y_{J}, \quad J \in \mathcal{D} .
$$

Then $\mathcal{B}_{R}, R \in \mathcal{D} \otimes \mathcal{D}$ satisfies Capon's local product condition (P1)- P4) with constants $C_{X}=C_{Y}=\kappa$, i.e. the following four properties (P1), (P2), (P3) and (P4) hold true:

(P1) For all $R \in \mathcal{D} \otimes \mathcal{D}$ the collection $\mathcal{B}_{R}$ consists of pairwise disjoint dyadic rectangles, and for all $R_{0}, R_{1} \in \mathcal{D} \otimes \mathcal{D}$ with $R_{0} \neq R_{1}$ we have $\mathcal{B}_{R_{0}} \cap \mathcal{B}_{R_{1}}=\emptyset$.

(P2) For all $I, J, I_{0}, J_{0}, I_{1}, J_{1} \in \mathcal{D}$ with $I_{0} \cap I_{1}=\emptyset, I_{0} \cup I_{1} \subset I$ and $J_{0} \cap J_{1}=\emptyset$, $J_{0} \cup J_{1} \subset J$ we have

$$
\begin{aligned}
X_{I_{0}} \cap X_{I_{1}} & =\emptyset, & X_{I_{0}} \cup X_{I_{1}} \subset X_{I}, \\
Y_{J_{0}} \cap Y_{J_{1}} & =\emptyset, & Y_{J_{0}} \cup Y_{J_{1}} \subset Y_{J} .
\end{aligned}
$$

(P3) For every $I, J \in \mathcal{D}$ we have

$$
\kappa^{-1}|I| \leq\left|X_{I}\right| \leq \kappa|I| \quad \text { and } \quad \kappa^{-1}|J| \leq\left|Y_{J}\right| \leq \kappa|J| .
$$

(P4) For all $I_{0}, J_{0}, I, J \in \mathcal{D}$ with $I_{0} \subset I, J_{0} \subset J$ and for every $K \in X_{I}, L \in y_{J}$, we have

$$
\frac{\left|K \cap X_{I_{0}}\right|}{|K|} \geq \kappa^{-1} \frac{\left|X_{I_{0}}\right|}{\left|X_{I}\right|} \quad \text { and } \quad \frac{\left|L \cap Y_{J_{0}}\right|}{|L|} \geq \kappa^{-1} \frac{\left|Y_{J_{0}}\right|}{\left|Y_{J}\right|} .
$$

Proof. (ㅍ1)-(

Remark 4.2. The conditions (P1) $-(\mathrm{P} 4)$ were introduced in [5] in a more general form: the collections $\mathcal{B}_{I \times J}, I, J \in \mathcal{D}$ in [5] have local product structure, i.e. there exist collections $X_{I \times J}, y_{I \times J}, I, J \in \mathcal{D}$ such that

$$
\mathcal{B}_{I \times J}=\left\{K \times L: K \in X_{I \times J}, L \in y_{I \times J}\right\}, \quad I, J \in \mathcal{D} .
$$

In Lemma 4.1, we have a special case of (4.2): true product structure (see (4.1)). To highlight the distinction explicitly, in Lemma 4.1 we have that $x_{I \times J}$ does not depend on $J$ and that $y_{I \times J}$ does not depend on $I$.

Lemma 4.3. Let $X$ and $y$ each a denote non-empty, finite collection of pairwise disjoint dyadic intervals, and define $X=\bigcup X$ as well as $Y=\bigcup y$. Given $\theta, \varepsilon \in$ $\{ \pm 1\}^{\mathcal{D}}$, put

$$
b^{(\theta, \varepsilon)}=\sum_{\substack{K \in \mathcal{X} \\ L \in \mathcal{Y}}} \theta_{K} \varepsilon_{L} h_{K \times L} .
$$

Then:

$$
\left\|b^{(\theta, \varepsilon)}\right\|_{H^{p}\left(H^{q}\right)}=|X|^{1 / p}|Y|^{1 / q} \quad \text { and } \quad\left\|b^{(\theta, \varepsilon)}\right\|_{\left(H^{p}\left(H^{q}\right)\right)^{*}}=|X|^{1 / p^{\prime}}|Y|^{1 / q^{\prime}},
$$

where $1 \leq p, q<\infty, 1<p^{\prime}, q^{\prime} \leq \infty$ with $\frac{1}{p}+\frac{1}{p^{\prime}}=\frac{1}{q}+\frac{1}{q^{\prime}}=1$. 
Lemma 4.3 follows immediately from [5, Lemma 4.1]. Since the proof is short, we include it here for the sake of completeness.

Proof. (4.3) and the disjointness of the collections $x, y$ yields

$$
\begin{aligned}
\left\|b^{(\theta, \varepsilon)}\right\|_{H^{p}\left(H^{q}\right)} & =\left(\int_{0}^{1}\left(\int_{0}^{1} \sum_{\substack{K \in \mathcal{X} \\
L \in \mathcal{Y}}} h_{K}^{2}(x) h_{L}^{2}(y) \mathrm{d} y\right)^{p / q} \mathrm{~d} x\right)^{1 / p} \\
& =|Y|^{1 / q}\left(\int_{0}^{1} \sum_{K \in \mathcal{X}} h_{K}^{2}(x) \mathrm{d} x\right)^{1 / p}=|X|^{1 / p}|Y|^{1 / q} .
\end{aligned}
$$

We will now compute $\left\|b^{(\theta, \varepsilon)}\right\|_{\left(H^{p}\left(H^{q}\right)\right)^{*}}$. To this end, let $h \in H^{p}\left(H^{q}\right)$ be given by $h=\sum_{K, L} a_{K \times L} h_{K \times L} \in H^{p}\left(H^{q}\right)$, and observe that by Hölder's inequality we obtain

$$
\begin{aligned}
\left\langle b^{(\theta, \varepsilon)}, h\right\rangle & \leq \sum_{K \in X}|K| \sum_{L \in \mathcal{Y}}\left|a_{K \times L}\right||L| \leq|Y|^{1 / q^{\prime}} \sum_{K \in \mathcal{X}}|K|\left(\sum_{L \in \mathcal{Y}}\left|a_{K \times L}\right|^{q}|L|\right)^{1 / q} \\
& \leq|X|^{1 / p^{\prime}}|Y|^{1 / q^{\prime}}\left(\sum_{K \in \mathcal{X}}|K|\left(\sum_{L \in \mathcal{Y}}\left|a_{K \times L}\right|^{q}|L|\right)^{p / q}\right)^{1 / p} \\
& =|X|^{1 / p^{\prime}}|Y|^{1 / q^{\prime}}\|h\|_{H^{p}\left(H^{q}\right)} .
\end{aligned}
$$

Thus, we have $\left\|b^{(\theta, \varepsilon)}\right\|_{\left(H^{p}\left(H^{q}\right)\right)^{*}} \leq|X|^{1 / p^{\prime}}|Y|^{1 / q^{\prime}}$. Since $\left\langle b^{(\theta, \varepsilon)}, b^{(\theta, \varepsilon)}\right\rangle=|X||Y|$ and $\left\|b^{(\theta, \varepsilon)}\right\|_{H^{p}\left(H^{q}\right)}=|X|^{1 / p}|Y|^{1 / q}$ by the first part of the proof, we obtain

$$
\left\|b^{(\theta, \varepsilon)}\right\|_{\left(H^{p}\left(H^{q}\right)\right)^{*}}=|X|^{1 / p^{\prime}}|Y|^{1 / q^{\prime}} .
$$

The following Theorem 4.4 is one of the two main ingredients in the proof of Theorem 3.1 the other one is the almost-diagonalization of operators using random block bases (see Theorem 4.5).

Theorem 4.4. Let $x_{I} \subset \mathcal{D}, I \in \mathcal{D}$ and $y_{J} \subset \mathcal{D}, J \in \mathcal{D}$ both satisfy condition (J) with constant $\kappa=1$, and define the product collections

$$
\mathcal{B}_{I \times J}=x_{I} \otimes y_{J}=\left\{K \times L: K \in x_{I}, L \in y_{J}\right\}, \quad I, J \in \mathcal{D} .
$$

Given $\theta, \varepsilon \in\{ \pm 1\}^{\mathcal{D}}$, we define the tensor product system

$$
b_{I \times J}^{(\theta, \varepsilon)}=f_{I}^{(\theta)} \otimes g_{J}^{(\varepsilon)}, \quad I, J \in \mathcal{D},
$$

where

$$
f_{I}^{(\theta)}=\sum_{K \in \mathcal{X}_{I}} \theta_{K} h_{K}, \quad I \in \mathcal{D} \quad \text { and } \quad g_{J}^{(\varepsilon)}=\sum_{L \in \mathcal{Y}_{J}} \varepsilon_{L} h_{L}, \quad J \in \mathcal{D} .
$$

Given $1 \leq p, q<\infty$, let $V$ denote either $H^{p}\left(H^{q}\right)$ or $\left(H^{p}\left(H^{q}\right)\right)^{*}$. Then the operators $B^{(\theta, \varepsilon)}, A^{(\theta, \varepsilon)}: V \rightarrow V$ given by

$$
B^{(\theta, \varepsilon)} f=\sum_{R \in \mathcal{D} \otimes \mathcal{D}} \frac{\left\langle f, h_{R}\right\rangle}{\left\|h_{R}\right\|_{2}^{2}} b_{R}^{(\theta, \varepsilon)} \quad \text { and } \quad A^{(\theta, \varepsilon)} f=\sum_{R \in \mathcal{D} \otimes \mathcal{D}} \frac{\left\langle f, b_{R}^{(\theta, \varepsilon)}\right\rangle}{\left\|b_{R}^{(\theta, \varepsilon)}\right\|_{2}^{2}} h_{R}
$$

satisfy the estimates

$$
\begin{gathered}
\left\|B^{(\theta, \varepsilon)} f\right\|_{V} \leq\|f\|_{V}, \quad f \in V, \\
\left\|A^{(\theta, \varepsilon)} f\right\|_{V} \leq\|f\|_{V}, \quad f \in V .
\end{gathered}
$$

Moreover, the diagram

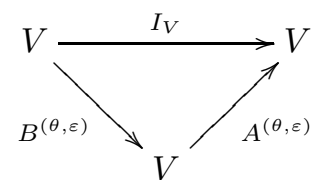


is commutative and the composition $P^{(\theta, \varepsilon)}=B^{(\theta, \varepsilon)} A^{(\theta, \varepsilon)}$ is the norm 1 projection $P^{(\theta, \varepsilon)}: V \rightarrow V$ given by

$$
P^{(\theta, \varepsilon)}(f)=\sum_{R \in \mathcal{D} \otimes \mathcal{D}} \frac{\left\langle f, b_{R}^{(\theta, \varepsilon)}\right\rangle}{\left\|b_{R}\right\|_{2}^{2}} b_{R}^{(\theta, \varepsilon)} .
$$

Consequently, the range of $B^{(\theta, \varepsilon)}$ is complemented (by $\left.P^{(\theta, \varepsilon)}\right)$, and $B^{(\theta, \varepsilon)}$ is an isometric isomorphism onto its range.

Proof. The case $V=H^{p}\left(H^{q}\right)$ follows immediately from Lemma 4.1] and [5].

If $V=\left(H^{p}\left(H^{q}\right)\right)^{*}$ the theorem follows from the case $V=H^{p}\left(H^{q}\right)$ and the observation that $\left(B^{(\theta, \varepsilon)}\right)^{*}=A^{(\theta, \varepsilon)}$ and $\left(A^{(\theta, \varepsilon)}\right)^{*}=B^{(\theta, \varepsilon)}$.

4.2. Random block bases with tensor product structure. Let $\mathbb{P}_{\theta}$ denote the uniform measure on $\Omega_{\theta}=\{ \pm 1\}^{\mathcal{D}}$, and let $\left(\Omega_{\varepsilon}, \mathbb{P}_{\varepsilon}\right)$ denote an independent copy of $\left(\Omega_{\theta}, \mathbb{P}_{\theta}\right) . \mathbb{P}_{\theta, \varepsilon}$ is the product measure on $\Omega_{\theta} \times \Omega_{\varepsilon}$. Moreover, $\mathbb{E}_{\theta}, \mathbb{E}_{\varepsilon}$ and $\mathbb{E}_{\theta, \varepsilon}$ are the expectations with respect to the probability measures $\mathbb{P}_{\theta}, \mathbb{P}_{\varepsilon}$ and $\mathbb{P}_{\theta, \varepsilon}$, respectively.

Given $n, N \in \mathbb{N}, I, J \in \mathcal{D}_{\leq n}$ and $x_{I}, y_{J} \subset \mathcal{D}_{\leq N}$, define the functions

$$
f_{I}^{(\theta)}=\sum_{K \in X_{I}} \theta_{K} h_{K}, \quad \theta \in \Omega_{\theta} \quad \text { and } \quad g_{J}^{(\varepsilon)}=\sum_{L \in \mathcal{Y}_{J}} \varepsilon_{L} h_{L}, \quad \varepsilon \in \Omega_{\varepsilon} .
$$

Hence, their tensor product $b_{I \times J}^{(\theta, \varepsilon)}$ is given by

$$
b_{I \times J}^{(\theta, \varepsilon)}=f_{I}^{(\theta)} \otimes g_{J}^{(\varepsilon)}=\sum_{\substack{K \in \mathcal{X}_{I} \\ L \in \mathcal{Y}_{J}}} \theta_{K} \varepsilon_{L} h_{K \times L}, \quad(\theta, \varepsilon) \in \Omega_{\theta} \times \Omega_{\varepsilon} .
$$

Let $1 \leq p, q<\infty$ and let $V_{N}$ denote either $H_{N}^{p}\left(H_{N}^{q}\right)$ or $\left(H_{N}^{p}\left(H_{N}^{q}\right)\right)^{*}$. Given a bounded linear operator $T: V_{N} \rightarrow V_{N}$, we put

$$
\begin{aligned}
W_{I, I^{\prime}, J, J^{\prime}}(\theta, \varepsilon) & =\left\langle T b_{I \times J}^{(\theta, \varepsilon)}, b_{I^{\prime} \times J^{\prime}}^{(\theta, \varepsilon)}\right\rangle, \quad I, J, I^{\prime}, J^{\prime} \in \mathcal{D}_{\leq n}, I \neq I^{\prime}, J \neq J^{\prime}, \\
X_{I, I^{\prime}, J}(\theta, \varepsilon) & =\left\langle T b_{I \times J}^{(\theta, \varepsilon)}, b_{I^{\prime} \times J^{\prime}}^{(\theta, \varepsilon)}\right\rangle, \quad I, J, I^{\prime} \in \mathcal{D}_{\leq n}, I \neq I^{\prime}, \\
Y_{I, J, J^{\prime}}(\theta, \varepsilon) & =\left\langle T b_{I \times J}^{(\theta, \varepsilon)}, b_{I^{\prime} \times J^{\prime}}^{(\theta, \varepsilon)}\right\rangle, \quad I, J, J^{\prime} \in \mathcal{D}_{\leq n}, J \neq J^{\prime}, \\
Z_{I, J}(\theta, \varepsilon) & =\left\langle T b_{I \times J}^{(\theta, \varepsilon)}, b_{I \times J}^{(\theta, \varepsilon)}\right\rangle-\sum_{\substack{K \in \mathcal{X}_{I} \\
L \in \mathcal{Y} J}}\left\langle T h_{K \times L}, h_{K \times L}\right\rangle, \quad I, J \in \mathcal{D}_{\leq n},
\end{aligned}
$$

for all $(\theta, \varepsilon) \in \Omega_{\theta} \times \Omega_{\varepsilon}$. From here on, we will regularly omit the subindices of the above random variables, i.e. $W=W_{I, I^{\prime}, J, J^{\prime}}, X=X_{I, I^{\prime}, J}, Y=Y_{I, J, J^{\prime}}$ and $Z=Z_{I, J}$.

Theorem 4.5. Given $n, N \in \mathbb{N}$, let $X_{I} \subset \mathcal{D}_{\leq N}, I \in \mathcal{D}_{\leq n}$ and $y_{J} \subset \mathcal{D}_{\leq N}, J \in \mathcal{D}_{\leq n}$ both denote non-empty collections which satisfy (J1). Define $\alpha>0$ by putting

$$
\alpha=\max \left\{|K|,|L|: K \in \mathcal{X}_{I}, L \in \mathcal{Y}_{J}, I, J \in \mathcal{D}_{\leq n}\right\} .
$$

Given $1 \leq p, q<\infty$, let $V_{N}$ denote either $H_{N}^{p}\left(H_{N}^{q}\right)$ or $\left(H_{N}^{p}\left(H_{N}^{q}\right)\right)^{*}$. Then for any bounded operator $T: V_{N} \rightarrow V_{N}$ we have

$$
\mathbb{E}_{\theta, \varepsilon} W=\mathbb{E}_{\theta, \varepsilon} X=\mathbb{E}_{\theta, \varepsilon} Y=\mathbb{E}_{\theta, \varepsilon} Z=0
$$

as well as the estimates

$$
\begin{aligned}
\mathbb{E}_{\theta, \varepsilon} W^{2} \leq\|T\|^{2} \alpha^{1 / 2}, & & \mathbb{E}_{\theta, \varepsilon} X^{2} \leq 4\|T\|^{2} \alpha^{1 / 2}, \\
\mathbb{E}_{\theta, \varepsilon} Y^{2} \leq 4\|T\|^{2} \alpha^{1 / 2}, & & \mathbb{E}_{\theta, \varepsilon} Z^{2} \leq 12\|T\|^{2} \alpha^{1 / 2},
\end{aligned}
$$

where the random variables $W, X, Y, Z$ are defined in (4.14).

The proof is given in Section 6 . 


\section{Proof of the main result Theorem 3.1}

Here we prove our main result Theorem 3.1 by extending the probabilistic method introduced in [8, Theorem 3.1] for one-parameter Hardy spaces $H^{p}$, to the biparameter Hardy spaces $H_{N}^{p}\left(H_{N}^{q}\right)$. The proof heavily relies on the results of Section 4 .

For convenience of the reader we repeat Theorem 3.1 here.

Theorem (Main result Theorem 3.1). Let $1 \leq p, q<\infty$, and let $\left(V_{k}: k \in \mathbb{N}_{0}\right)$ denote either

$$
\left(H_{k}^{p}\left(H_{k}^{q}\right): k \in \mathbb{N}_{0}\right) \quad \text { or } \quad\left(\left(H_{k}^{p}\left(H_{k}^{q}\right)\right)^{*}: k \in \mathbb{N}_{0}\right) .
$$

Let $n \in \mathbb{N}_{0}$ and $\delta, \Gamma, \eta>0$. Define the integer $N=N(n, \delta, \Gamma, \eta)$ by the formula

$$
N=41(n+3)+\left\lfloor 4 \log _{2}(\Gamma / \delta)+4 \log _{2}\left(1+\eta^{-1}\right)\right\rfloor .
$$

Then for any operator $T: V_{N} \rightarrow V_{N}$ satisfying

$$
\|T\| \leq \Gamma \quad \text { and } \quad\left|\left\langle T h_{Q}, h_{Q}\right\rangle\right| \geq \delta|Q|, \quad Q \in \mathcal{D}_{\leq N} \otimes \mathcal{D}_{\leq N},
$$

there exist bounded linear operators $E: V_{n} \rightarrow V_{N}$ and $F: V_{N} \rightarrow V_{n}$, such that the diagram

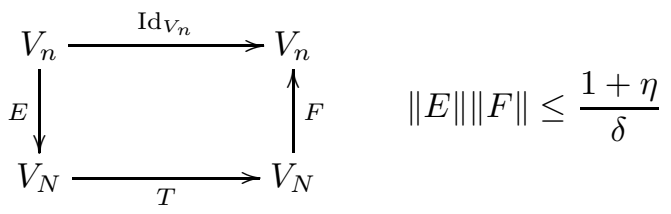

is commutative.

Proof. Let $M: V_{N} \rightarrow V_{N}$ denote the norm 1 multiplication operator given by the linear extension of

$$
h_{Q} \mapsto \operatorname{sign}\left(\left\langle T h_{Q}, h_{Q}\right\rangle\right) h_{Q}, \quad Q \in \mathcal{D}_{\leq N} \otimes \mathcal{D}_{\leq N} .
$$

By (5.3), we obtain

$$
\left\langle T M h_{Q}, h_{Q}\right\rangle=\left|\left\langle T h_{Q}, h_{Q}\right\rangle\right| \geq \delta|Q|, \quad Q \in \mathcal{D}_{\leq N} \otimes \mathcal{D}_{\leq N},
$$

and therefore we can assume

$$
\left\langle T h_{Q}, h_{Q}\right\rangle \geq \delta|Q|, \quad Q \in \mathcal{D}_{\leq N} \otimes \mathcal{D}_{\leq N} .
$$

Before we proceed to Step 1 of the proof, we define the constants $m_{0}$ and $\eta_{0}$ : Let $m_{0} \in \mathbb{N}_{0}$ denote the smallest integer such that

$$
2^{m_{0}}>\frac{2^{8(n+3)} \Gamma^{4}}{\eta_{0}^{4}}, \quad \text { where } \quad \eta_{0}=\frac{\eta \delta}{(1+\eta) 2^{8(n+2)}} .
$$

Step 1: COnstructing The Block BAsis $b_{R}^{(\theta, \varepsilon)}, R \in \mathcal{D}_{\leq n} \otimes \mathcal{D}_{\leq n}$. In this step, we will define a random block basis $(\theta, \varepsilon) \mapsto b_{R}^{(\theta, \varepsilon)}, R \in \overline{\mathcal{D}}_{\leq n} \otimes \overline{\mathcal{D}}_{\leq n}$ of the Haar system $h_{Q}, Q \in \mathcal{D}_{\leq N} \otimes \mathcal{D}_{\leq N}$ given by

$$
b_{I \times J}^{(\theta, \varepsilon)}=f_{I}^{(\theta)} \otimes g_{J}^{(\varepsilon)}=\sum_{K \in X_{I}} \theta_{K} h_{K} \otimes \sum_{L \in \mathcal{Y}_{J}} \varepsilon_{L} h_{L}, \quad \theta \in \Omega_{\theta}, \varepsilon \in \Omega_{\varepsilon},
$$

where $x_{I} \subset \mathcal{D}_{\leq N}, I \in \mathcal{D}_{\leq n}$ and $y_{J} \subset \mathcal{D}_{\leq N}, J \in \mathcal{D}_{\leq n}$ both satisfy condition (J) with constant $\kappa=1$. The collections will be selected by a minimalist GamlenGaudet construction. Then, using Theorem 4.5, we will find signs $(\theta, \varepsilon) \in \Omega_{\theta} \times \Omega_{\varepsilon}$ such that

$$
\begin{array}{rrr}
\left|\left\langle T b_{R}^{(\theta, \varepsilon)}, b_{R^{\prime}}^{(\theta, \varepsilon)}\right\rangle\right| & \leq \eta_{0}, & R, R^{\prime} \in \mathcal{D}_{\leq n} \otimes \mathcal{D}_{\leq n}, \quad R \neq R^{\prime} \\
\left\langle T b_{R}^{(\theta, \varepsilon)}, b_{R}^{(\theta, \varepsilon)}\right\rangle & \geq\left(\delta-\eta_{0} 2^{2 n}\right)\left\|b_{R}^{(\theta, \varepsilon)}\right\|_{2}^{2}, & R \in \mathcal{D}_{\leq n} \otimes \mathcal{D}_{\leq n} .
\end{array}
$$


We will now inductively define the collections $x_{I}, I \in \mathcal{D}_{\leq n}$ and $y_{J}, J \in \mathcal{D}_{\leq n}$. We begin by putting,

$$
x_{[0,1)}=y_{[0,1)}=\mathcal{D}_{m_{0}} .
$$

Now, let $0 \leq k \leq n-1$, assume that we have already constructed the collections $x_{I}, I \in \mathcal{D}_{\leq k}$ and $y_{J}, J \in \mathcal{D}_{\leq k}$. Then we define

$$
\begin{array}{lll}
x_{I^{+}}=\left\{K^{+}: K \in \mathcal{X}_{I}\right\}, & x_{I^{-}}=\left\{K^{-}: K \in \mathcal{X}_{I}\right\}, & I \in \mathcal{D}_{k}, \\
y_{J^{+}}=\left\{K^{+}: K \in \mathcal{y}_{J}\right\}, & y_{J^{-}}=\left\{K^{-}: K \in \mathcal{y}_{J}\right\}, & J \in \mathcal{D}_{k} .
\end{array}
$$

Clearly, $x_{I}, I \in \mathcal{D}_{\leq n}$ and $y_{J}, J \in \mathcal{D}_{\leq n}$ both satisfy condition (J) with constant $\kappa=1$.

Next, we will use the probabilistic Theorem 4.5 to find $\operatorname{signs}(\theta, \varepsilon) \in \Omega_{\theta} \times \Omega_{\varepsilon}$ such that (5.8) is satisfied. To this end, we define the off-diagonal events

$$
O_{R, R^{\prime}}=\left\{(\theta, \varepsilon):\left|\left\langle T b_{R}^{(\theta, \varepsilon)}, b_{R^{\prime}}^{(\theta, \varepsilon)}\right\rangle\right|>\eta_{0}\right\}, \quad R, R^{\prime} \in \mathcal{D}_{\leq n} \otimes \mathcal{D}_{\leq n}, R \neq R^{\prime}
$$

and the diagonal events

$$
D_{I, J}=\left\{(\theta, \varepsilon):\left|\left\langle T b_{I \times J}^{(\theta, \varepsilon)}, b_{I \times J}^{(\theta, \varepsilon)}\right\rangle-\sum_{\substack{K \in \mathcal{X}_{I} \\ L \in \mathcal{Y}_{J}}}\left\langle T h_{K \times L}, h_{K \times L}\right\rangle\right|>\eta_{0}\right\}, \quad I, J \in \mathcal{D}_{\leq n} .
$$

By Theorem 4.5 and the definition of the random variables $W, X, Y, Z$ (see (4.14) $)$, we obtain

$$
\begin{array}{rr}
\mathbb{P}_{\theta, \varepsilon}\left(O_{R, R^{\prime}}\right) \leq \frac{4 \Gamma^{2}}{2^{m_{0} / 2} \eta_{0}^{2}}, & R, R^{\prime} \in \mathcal{D}_{\leq n} \otimes \mathcal{D}_{\leq n}, R \neq R^{\prime}, \\
\mathbb{P}_{\theta, \varepsilon}\left(D_{I, J}\right) \leq \frac{12 \Gamma^{2}}{2^{m_{0} / 2} \eta_{0}^{2}}, & I, J \in \mathcal{D}_{\leq n} .
\end{array}
$$

Combining (5.11) with (5.6) yields

$$
\mathbb{P}_{\theta, \varepsilon}\left(\bigcup_{\substack{R, R^{\prime} \in \mathcal{D}_{\leq n} \otimes \mathcal{D} \leq n \\ R \neq R^{\prime}}} O_{R, R^{\prime}} \cup \bigcup_{I, J \in \mathcal{D}_{\leq n}} D_{I, J}\right) \leq 2^{4(n+3)} \frac{\Gamma^{2}}{2^{m_{0} / 2} \eta_{0}^{2}}<1
$$

Hence, we can find at least one $(\theta, \varepsilon) \in \Omega_{\theta} \times \Omega_{\varepsilon}$ such that

$$
\begin{array}{r}
\left|\left\langle T b_{R}^{(\theta, \varepsilon)}, b_{R^{\prime}}^{(\theta, \varepsilon)}\right\rangle\right| \leq \eta_{0}, \quad R, R^{\prime} \in \mathcal{D}_{\leq n} \otimes \mathcal{D}_{\leq n}, \quad R \neq R^{\prime}, \\
\left|\left\langle T b_{I \times J}^{(\theta, \varepsilon)}, b_{I \times J}^{(\theta, \varepsilon)}\right\rangle-\sum_{\substack{K \in \mathcal{X}_{I} \\
L \in \mathcal{Y}_{J}}}\left\langle T h_{K \times L}, h_{K \times L}\right\rangle\right| \leq \eta_{0}, \quad I, J \in \mathcal{D}_{\leq n} .
\end{array}
$$

Recall that $\kappa=1$ by construction of $x_{I}$ and $y_{J}, I, J \in \mathcal{D}_{\leq n}$ (see (5.10)). Hence, by (5.3), (J1) and (J3) we obtain

$$
\sum_{\substack{K \in \mathcal{X}_{I} \\ L \in \mathcal{Y}_{J}}}\left\langle T h_{K \times L}, h_{K \times L}\right\rangle \geq \sum_{\substack{K \in \mathcal{X}_{I} \\ L \in \mathcal{Y}_{J}}} \delta|K \times L|=\delta\left|X_{I} \times Y_{J}\right|=\delta|I \times J|, \quad I, J \in \mathcal{D}_{\leq n} .
$$

The latter estimate and (5.13b) give us

$$
\left\langle T b_{R}^{(\theta, \varepsilon)}, b_{R}^{(\theta, \varepsilon)}\right\rangle \geq \delta|R|-\eta_{0}, \quad R \in \mathcal{D}_{\leq n} \otimes \mathcal{D}_{\leq n} .
$$

Note that by Lemma 4.3 we have $\left\|b_{R}^{(\theta, \varepsilon)}\right\|_{2}^{2}=R$, thus we obtain from (5.14)

$$
\left\langle T b_{R}^{(\theta, \varepsilon)}, b_{R}^{(\theta, \varepsilon)}\right\rangle \geq\left(\delta-\eta_{0} 2^{2 n}\right)\left\|b_{R}^{(\theta, \varepsilon)}\right\|_{2}^{2}, \quad R \in \mathcal{D}_{\leq n} \otimes \mathcal{D}_{\leq n} .
$$

Combining (5.13a) with (5.15) yields

$$
\begin{array}{rr}
\left|\left\langle T b_{R}^{(\theta, \varepsilon)}, b_{R^{\prime}}^{(\theta, \varepsilon)}\right\rangle\right| \leq \eta_{0}, & R, R^{\prime} \in \mathcal{D}_{\leq n} \otimes \mathcal{D}_{\leq n}, R \neq R^{\prime} \\
\left\langle T b_{R}^{(\theta, \varepsilon)}, b_{R}^{(\theta, \varepsilon)}\right\rangle \geq\left(\delta-\eta_{0} 2^{2 n}\right)\left\|b_{R}^{(\theta, \varepsilon)}\right\|_{2}^{2}, & R \in \mathcal{D}_{\leq n} \otimes \mathcal{D}_{\leq n} .
\end{array}
$$


Step 2: Constructing the operators. Here, we will use the basic operators $B^{(\theta, \varepsilon)}: V_{n} \rightarrow V_{N}$ and $A^{(\theta, \varepsilon)}: V_{N} \rightarrow V_{n}$ given by

$$
\begin{array}{ll}
B^{(\theta, \varepsilon)} f=\sum_{R \in \mathcal{D}_{\leq n} \otimes \mathcal{D}_{\leq n}} \frac{\left\langle f, h_{R}\right\rangle}{\left\|h_{R}\right\|_{2}^{2}} b_{R}^{(\theta, \varepsilon)}, & f \in V_{n}, \\
A^{(\theta, \varepsilon)} f=\sum_{R \in \mathcal{D}_{\leq n} \otimes \mathcal{D}_{\leq n}} \frac{\left\langle f, b_{R}^{(\theta, \varepsilon)}\right\rangle}{\left\|b_{R}^{(\theta, \varepsilon)}\right\|_{2}^{2}} h_{R}, & f \in V_{N},
\end{array}
$$

as building blocks for the operators $E$ and $F$ in diagram (5.4). Let us recall that by Theorem 4.4, the operators $B^{(\theta, \varepsilon)}$ and $A^{(\theta, \varepsilon)}$ satisfy the estimates

$$
\left\|B^{(\theta, \varepsilon)}\right\| \leq 1 \quad \text { and } \quad\left\|A^{(\theta, \varepsilon)}\right\| \leq 1
$$

and $P^{(\theta, \varepsilon)}: V_{N} \rightarrow V_{N}$ defined as $P^{(\theta, \varepsilon)}=B^{(\theta, \varepsilon)} A^{(\theta, \varepsilon)}$ is a norm 1 projection given by

$$
P^{(\theta, \varepsilon)} f=\sum_{R \in \mathcal{D} \leq n \otimes \mathcal{D} \leq n} \frac{\left\langle f, b_{R}^{(\theta, \varepsilon)}\right\rangle}{\left\|b_{R}^{(\theta, \varepsilon)}\right\|_{2}^{2}} b_{R}^{(\theta, \varepsilon)}, \quad f \in V_{N} .
$$

Now put $Y=P^{(\theta, \varepsilon)}\left(V_{N}\right)$ and note that the following diagram is commutative:

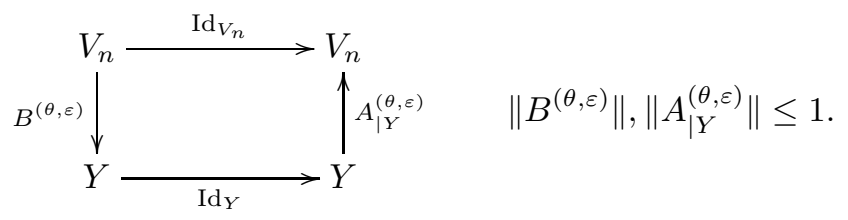

Observe that $T$ almost acts as a multiplication operator on $Y$ (see (5.16) $)$ ). Next, we define $U^{(\theta, \varepsilon)}: V_{N} \rightarrow Y$ by putting

$$
U^{(\theta, \varepsilon)} f=\sum_{R \in \mathcal{D}_{\leq n} \otimes \mathcal{D}_{\leq n}} \frac{\left\langle f, b_{R}^{(\theta, \varepsilon)}\right\rangle}{\left\langle T b_{R}^{(\theta, \varepsilon)}, b_{R}^{(\theta, \varepsilon)}\right\rangle} b_{R}^{(\theta, \varepsilon)}, \quad f \in V_{N} .
$$

By the 1-unconditionality of the bi-parameter Haar system in $V_{N}$ and the definition of the norm 1 projection $P^{(\theta, \varepsilon)}$ (see (5.19) and (5.18) ), we obtain

$$
\left\|U^{(\theta, \varepsilon)}\right\| \leq \frac{\left\|P^{(\theta, \varepsilon)}\right\|}{\delta-\eta_{0} 2^{2 n}} \leq \frac{1}{\delta-\eta_{0} 2^{2 n}} .
$$

We will now show that $U^{(\theta, \varepsilon)}: V_{N} \rightarrow Y$ almost acts as an inverse of $T$ restricted to $Y$. Firstly, for all $g=\sum_{R \in \mathcal{D}_{\leq n} \otimes \mathcal{D}_{\leq n}} a_{R} b_{R}^{(\theta, \varepsilon)} \in Y$, we have the following identity:

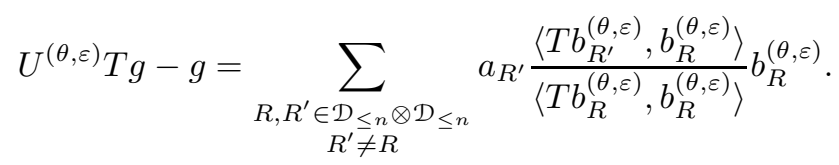

Secondly, by Lemma 4.3, we have the estimate

$$
\left|a_{R^{\prime}}\right| \leq \frac{\|g\|_{V_{N}}}{\left\|b_{R^{\prime}}^{(\theta, \varepsilon)}\right\|_{V_{N}}} \leq 2^{2(n+1)}\|g\|_{V_{N}}, \quad R^{\prime} \in \mathcal{D}_{\leq n} \otimes \mathcal{D}_{\leq n},
$$

thus, by (5.23) and (5.16), we obtain

$$
\left\|U^{(\theta, \varepsilon)} T g-g\right\|_{V_{N}} \leq \frac{\eta_{0} 2^{8(n+1)}}{\delta-\eta_{0} 2^{2 n}}\|g\|_{V_{N}}
$$


Next, let $I: Y \rightarrow V_{N}$ denote the operator given by $I y=y$. Observe that by (5.6) we have $\frac{\eta_{0} 2^{8(n+1)}}{\delta-\eta_{0} 2^{2 n}}<1$, hence we obtain from (5.24) that

$$
\left\|\left(U^{(\theta, \varepsilon)} T I\right)^{-1} g\right\|_{V_{N}} \leq \frac{1}{1-\frac{\eta_{0} 2^{8(n+1)}}{\delta-\eta_{0} 2^{2 n}}}\|g\|_{V_{N}} .
$$

Now, define $S^{(\theta, \varepsilon)}: V_{N} \rightarrow Y$ by putting $S^{(\theta, \varepsilon)}=\left(U^{(\theta, \varepsilon)} T I\right)^{-1} U^{(\theta, \varepsilon)}$, and note that (5.22), (5.25) and (5.6) gives us

$$
\left\|S^{(\theta, \varepsilon)}\right\| \leq \frac{1}{\delta-\eta_{0}\left(2^{2 n}+2^{8(n+1)}\right)} \leq \frac{1+\eta}{\delta} .
$$

Moreover, the following diagram is commutative:

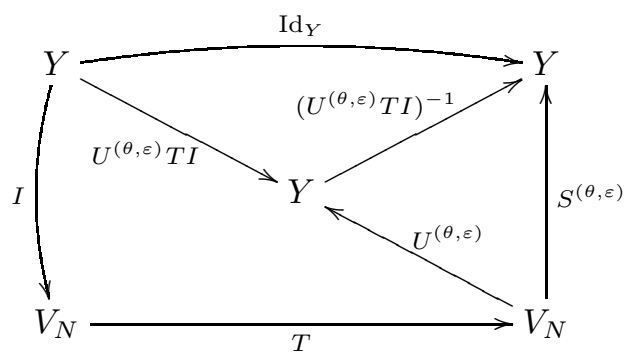

$$
\|I\|\left\|S^{(\theta, \varepsilon)}\right\| \leq \frac{1+\eta}{\delta}
$$

Merging the diagrams (5.20) and (5.26) yields

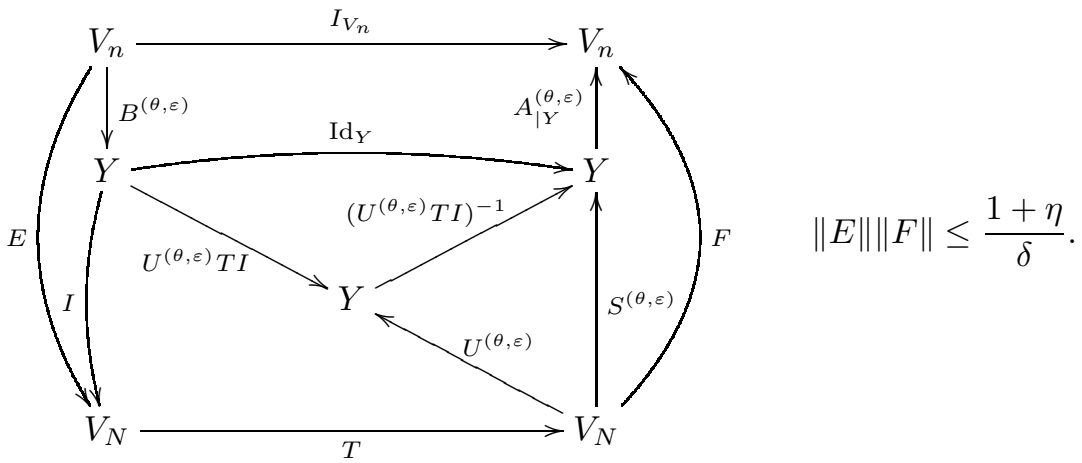

Finally, by reviewing the construction of our block basis $b_{R}^{(\theta)}, R \in \mathcal{D}_{\leq n} \otimes \mathcal{D}_{\leq n}$ (see (5.9) and (5.10) ), the definition of our basic operators $B^{(\theta, \varepsilon)}$ and $A^{(\theta, \varepsilon)}$ and the constants defined in (5.6), we conclude that (5.2) is an appropriate choice for $N$.

\section{Proof of Theorem 4.5}

We only present the proof for $V_{N}=H_{N}^{p}\left(H_{N}^{q}\right)$. For $V_{N}=\left(H_{N}^{p}\left(H_{N}^{q}\right)\right)^{*}$, the roles of $p, q$ and $p^{\prime}, q^{\prime}$ are reversed, where $\frac{1}{p}+\frac{1}{p^{\prime}}=1$ and $\frac{1}{q}+\frac{1}{q^{\prime}}=1$.

The proof is divided into four parts:

$\triangleright$ Estimates for $W$,

$\triangleright$ Estimates for $X$

$\triangleright$ Estimates for $Y$,

$\triangleright$ Estimates for $Z$.

For each of the four random variables $W, X, Y, Z$, there is a unique ensemble of summation parameters, which is recorded at the beginning of each section. The summation parameters are split into separate cases. Every case possess a left variant and a right variant, which refers to whether we place the sum in the left argument or in the right argument of the bilinear form. The estimates obtained for the left 
and the right variant of a case are combined to a single estimate at the end of each case.

Before we begin with the proof, we make the following crucial observations: Firstly, $\mathbb{E}_{\zeta} \zeta_{S_{0}} \zeta_{S_{1}} \zeta_{S_{0}^{\prime}} \zeta_{S_{1}^{\prime}} \in\{0,1\}$, for all $S_{0}, S_{1}, S_{0}^{\prime}, S_{1}^{\prime} \in \mathcal{D}$. Secondly, given dyadic intervals $S_{0}, S_{1}, S_{0}^{\prime}, S_{1}^{\prime} \in \mathcal{D}$, we have that $\mathbb{E}_{\zeta} \zeta_{S_{0}} \zeta_{S_{1}} \zeta_{S_{0}^{\prime}} \zeta_{S_{1}^{\prime}}=1$ if and only if one of the following conditions (R1)-( $\mathrm{R} 4)$ is satisfied:

(R1) $S_{0}=S_{1}=S_{0}^{\prime}=S_{1}^{\prime}$;

(R2) $S_{0}=S_{1} \neq S_{0}^{\prime}=S_{1}^{\prime}$;

(R3) $S_{0}=S_{0}^{\prime} \neq S_{1}=S_{1}^{\prime}$;

(R4) $S_{0}=S_{1}^{\prime} \neq S_{1}=S_{0}^{\prime}$.

6.1. Estimates for $W$. In this case, the following variables will always be summed over the following sets:

$$
\begin{aligned}
& \triangleright K_{0}, K_{1} \text { over } x_{I} ; \\
& \triangleright K_{0}^{\prime}, K_{1}^{\prime} \text { over } X_{I^{\prime}} ; \\
& \triangleright L_{0}, L_{1} \text { over } y_{J} ; \\
& \triangleright L_{0}^{\prime}, L_{1}^{\prime} \text { over } y_{J^{\prime}} .
\end{aligned}
$$

Proof. First, note that by (4.14a) and (4.13) we obtain $W^{2}(\theta, \varepsilon)$ is given by

$$
\sum_{\substack{K_{0}, K_{1}, K_{0}^{\prime}, K_{1}^{\prime} \\ L_{0}, L_{1}, L_{0}^{\prime}, L_{1}^{\prime}}} \theta_{K_{0}} \theta_{K_{1}} \theta_{K_{0}^{\prime}} \theta_{K_{1}^{\prime}} \varepsilon_{L_{0}} \varepsilon_{L_{1}} \varepsilon_{L_{0}^{\prime}} \varepsilon_{L_{1}^{\prime}}\left\langle T h_{K_{0} \times L_{0}}, h_{K_{0}^{\prime} \times L_{0}^{\prime}}\right\rangle\left\langle T h_{K_{1} \times L_{1}}, h_{K_{1}^{\prime} \times L_{1}^{\prime}}\right\rangle
$$

In view of (J1) and (R1)-( $\mathrm{R} 4)$, we obtain that

$$
\mathbb{E}_{\theta, \varepsilon} W^{2}=\sum_{K_{0}, K_{0}^{\prime}, L_{0}, L_{0}^{\prime}}\left\langle T h_{K_{0} \times L_{0}}, h_{K_{0}^{\prime} \times L_{0}^{\prime}}\right\rangle^{2}
$$

CASE 1: $K_{0}=K_{1} \neq K_{0}^{\prime}=K_{1}^{\prime}, L_{0}=L_{1} \neq L_{0}^{\prime}=L_{1}^{\prime}(((1)(1))((0)(0))(\mathrm{NIL}$ NIL)) - LEFT VARIANT. In this case, we have to estimate

$$
\sum_{K_{0}^{\prime}, L_{0}^{\prime}, K_{0}, L_{0}}\left\langle T h_{K_{0} \times L_{0}}, h_{K_{0}^{\prime} \times L_{0}^{\prime}}\right\rangle\left\langle T h_{K_{0} \times L_{0}}, h_{K_{0}^{\prime} \times L_{0}^{\prime}}\right\rangle .
$$

We put $a_{K_{0}, K_{0}^{\prime}, L_{0}, L_{0}^{\prime}}=\left\langle T h_{K_{0} \times L_{0}}, h_{K_{0}^{\prime} \times L_{0}^{\prime}}\right\rangle$ and note the estimate

$$
\left|a_{K_{0}, K_{0}^{\prime}, L_{0}, L_{0}^{\prime}}\right| \leq\|T\|\left|K_{0}\right|^{1 / p}\left|L_{0}\right|^{1 / q}\left|K_{0}^{\prime}\right|^{1 / p^{\prime}}\left|L_{0}^{\prime}\right|^{1 / q^{\prime}} .
$$

Now, we write (6.3) as follows:

$$
\sum_{K_{0}^{\prime}, L_{0}^{\prime}}\left\langle T \sum_{K_{0}, L_{0}} a_{K_{0}, K_{0}^{\prime}, L_{0}, L_{0}^{\prime}} h_{K_{0} \times L_{0}}, h_{K_{0}^{\prime} \times L_{0}^{\prime}}\right\rangle .
$$

By duality, we obtain the subsequent upper estimate for (6.5):

$$
\sum_{K_{0}^{\prime}, L_{0}^{\prime}}\left\|T \sum_{K_{0}, L_{0}} a_{K_{0}, K_{0}^{\prime}, L_{0}, L_{0}^{\prime}} h_{K_{0} \times L_{0}}\right\|_{H^{p}\left(H^{q}\right)}\left\|h_{K_{0}^{\prime} \times L_{0}^{\prime}}\right\|_{\left(H^{p}\left(H^{q}\right)\right)^{*}} \cdot
$$

Estimate (6.4) and the disjointness of the dyadic intervals (see (J1)) yield

$$
\sum_{K_{0}^{\prime}, L_{0}^{\prime}}\|T\|\left\|\sum_{K_{0}, L_{0}} \max _{K_{0}, L_{0}}\left(\|T\|\left|K_{0}\right|^{1 / p}\left|L_{0}\right|^{1 / q}\left|K_{0}^{\prime}\right|^{1 / p^{\prime}}\left|L_{0}^{\prime}\right|^{1 / q^{\prime}}\right) h_{K_{0} \times L_{0}}\right\|\left\|_{H^{p}\left(H^{q}\right)}\right\| h_{K_{0}^{\prime} \times L_{0}^{\prime}} \|_{\left(H^{p}\left(H^{q}\right)\right)^{*}} .
$$


Consequently, we obtain

$$
\sum_{K_{0}^{\prime}, L_{0}^{\prime}}\|T\| \max _{K_{0}, L_{0}}\left(\|T\|\left|K_{0}\right|^{1 / p}\left|L_{0}\right|^{1 / q}\left|K_{0}^{\prime}\right|^{1 / p^{\prime}}\left|L_{0}^{\prime}\right|^{1 / q^{\prime}}\right)\left\|\sum_{K_{0}, L_{0}} h_{K_{0} \times L_{0}}\right\|\left\|_{H^{p}\left(H^{q}\right)}\right\| h_{K_{0}^{\prime} \times L_{0}^{\prime}} \|_{\left(H^{p}\left(H^{q}\right)\right)^{*}} .
$$

Thus, (6.8) is bounded from above by

$$
\|T\|^{2} \max _{K_{0}, L_{0}}\left|K_{0}\right|^{1 / p}\left|L_{0}\right|^{1 / q} \sum_{K_{0}^{\prime}, L_{0}^{\prime}}\left|K_{0}^{\prime}\right|^{2 / p^{\prime}}\left|L_{0}^{\prime}\right|^{2 / q^{\prime}}
$$

which by Hölder's inequality is dominated by

$$
\|T\|^{2} \max _{K_{0}, L_{0}, K_{0}^{\prime}, L_{0}^{\prime}}\left|K_{0}\right|^{1 / p}\left|L_{0}\right|^{1 / q}\left|K_{0}^{\prime}\right|^{2 / p^{\prime}-1}\left|L_{0}^{\prime}\right|^{2 / q^{\prime}-1} .
$$

Inserting $\left|K_{0}\right|,\left|L_{0}\right|,\left|K_{0}^{\prime}\right|,\left|L_{0}^{\prime}\right| \leq \alpha$ (see (4.15)) into (6.9), we obtain the estimate

$$
\|T\|^{2} \alpha^{1 / p^{\prime}+1 / q^{\prime}}
$$

CASE 2: $K_{0}=K_{1} \neq K_{0}^{\prime}=K_{1}^{\prime}, L_{0}=L_{1} \neq L_{0}^{\prime}=L_{1}^{\prime}(((0)(0))($ NIL NIL $)((1)$ (1)) ) - RIGHT VARIANT. In this case, we have to estimate

$$
\sum_{K_{0}, L_{0}, K_{0}^{\prime}, L_{0}^{\prime}}\left\langle T h_{K_{0} \times L_{0}}, h_{K_{0}^{\prime} \times L_{0}^{\prime}}\right\rangle\left\langle T h_{K_{0} \times L_{0}}, h_{K_{0}^{\prime} \times L_{0}^{\prime}}\right\rangle .
$$

We put $a_{K_{0}, K_{0}^{\prime}, L_{0}, L_{0}^{\prime}}=\left\langle T h_{K_{0} \times L_{0}}, h_{K_{0}^{\prime} \times L_{0}^{\prime}}\right\rangle$ and note the estimate

$$
\left|a_{K_{0}, K_{0}^{\prime}, L_{0}, L_{0}^{\prime}}\right| \leq\|T\|\left|K_{0}\right|^{1 / p}\left|L_{0}\right|^{1 / q}\left|K_{0}^{\prime}\right|^{1 / p^{\prime}}\left|L_{0}^{\prime}\right|^{1 / q^{\prime}} .
$$

Now, we write (6.11) as follows:

$$
\sum_{K_{0}, L_{0}}\left\langle T h_{K_{0} \times L_{0}}, \sum_{K_{0}^{\prime}, L_{0}^{\prime}} a_{K_{0}, K_{0}^{\prime}, L_{0}, L_{0}^{\prime}} h_{K_{0}^{\prime} \times L_{0}^{\prime}}\right\rangle .
$$

By duality, we obtain the subsequent upper estimate for (6.13):

$$
\sum_{K_{0}, L_{0}}\left\|T h_{K_{0} \times L_{0}}\right\|_{H^{p}\left(H^{q}\right)}\left\|\sum_{K_{0}^{\prime}, L_{0}^{\prime}} a_{K_{0}, K_{0}^{\prime}, L_{0}, L_{0}^{\prime}} h_{K_{0}^{\prime} \times L_{0}^{\prime}}\right\|_{\left(H^{p}\left(H^{q}\right)\right)^{*}} .
$$

Estimate (6.12) and the disjointness of the dyadic intervals (see (J1) ) yield

$$
\sum_{K_{0}, L_{0}}\|T\|\left\|h_{K_{0} \times L_{0}}\right\|_{H^{p}\left(H^{q}\right)}\left\|\sum_{K_{0}^{\prime}, L_{0}^{\prime}} \max _{K_{0}^{\prime}, L_{0}^{\prime}}\left(\|T\|\left|K_{0}\right|^{1 / p}\left|L_{0}\right|^{1 / q}\left|K_{0}^{\prime}\right|^{1 / p^{\prime}}\left|L_{0}^{\prime}\right|^{1 / q^{\prime}}\right) h_{K_{0}^{\prime} \times L_{0}^{\prime}}\right\|_{\left(H^{p}\left(H^{q}\right)\right)^{*}} .
$$

Consequently, we obtain

$$
\sum_{K_{0}, L_{0}}\|T\|\left\|h_{K_{0} \times L_{0}}\right\|_{H^{p}\left(H^{q}\right)} \max _{K_{0}^{\prime}, L_{0}^{\prime}}\left(\|T\|\left|K_{0}\right|^{1 / p}\left|L_{0}\right|^{1 / q}\left|K_{0}^{\prime}\right|^{1 / p^{\prime}}\left|L_{0}^{\prime}\right|^{1 / q^{\prime}}\right)\left\|\sum_{K_{0}^{\prime}, L_{0}^{\prime}} h_{K_{0}^{\prime} \times L_{0}^{\prime}}\right\|_{\left(H^{p}\left(H^{q}\right)\right)^{*}} .
$$

Thus, 6.16) is bounded from above by

$$
\|T\|^{2} \max _{K_{0}^{\prime}, L_{0}^{\prime}}\left|K_{0}^{\prime}\right|^{1 / p^{\prime}}\left|L_{0}^{\prime}\right|^{1 / q^{\prime}} \sum_{K_{0}, L_{0}}\left|K_{0}\right|^{2 / p}\left|L_{0}\right|^{2 / q}
$$

which by Hölder's inequality is dominated by

$$
\|T\|^{2} \max _{K_{0}, L_{0}, K_{0}^{\prime}, L_{0}^{\prime}}\left|K_{0}^{\prime}\right|^{1 / p^{\prime}}\left|L_{0}^{\prime}\right|^{1 / q^{\prime}}\left|K_{0}\right|^{2 / p-1}\left|L_{0}\right|^{2 / q-1} .
$$


Inserting $\left|K_{0}\right|,\left|L_{0}\right|,\left|K_{0}^{\prime}\right|,\left|L_{0}^{\prime}\right| \leq \alpha$ (see (4.15)) into (6.17), we obtain the estimate

$$
\|T\|^{2} \alpha^{1 / p+1 / q} \text {. }
$$

Summary for $W$. Combining (6.10) with (6.18) yields

$$
\mathbb{E}_{\theta, \varepsilon} W^{2} \leq\|T\|^{2} \alpha
$$

6.2. Estimates for $X$. In this case, the following variables will always be summed over the following sets:

$$
\begin{aligned}
& \triangleright K_{0}, K_{1} \text { over } X_{I} ; \\
& \triangleright K_{0}^{\prime}, K_{1}^{\prime} \text { over } X_{I^{\prime}} ; \\
& \triangleright L_{0}, L_{1}, L^{\prime}, L_{0}^{\prime}, L_{1}^{\prime} \text { over } y_{J} .
\end{aligned}
$$

Proof. Note that by (4.14b) and (4.13) we obtain $X^{2}(\theta, \varepsilon)$ is given by

$$
\sum_{\substack{K_{0}, K_{1}, K_{0}^{\prime}, K_{1}^{\prime} \\ L_{0}, L_{1}, L_{0}^{\prime}, L_{1}^{\prime}}} \theta_{K_{0}} \theta_{K_{1}} \theta_{K_{0}^{\prime}} \theta_{K_{1}^{\prime}} \varepsilon_{L_{0}} \varepsilon_{L_{1}} \varepsilon_{L_{0}^{\prime}} \varepsilon_{L_{1}^{\prime}}\left\langle T h_{K_{0} \times L_{0}}, h_{K_{0}^{\prime} \times L_{0}^{\prime}}\right\rangle\left\langle T h_{K_{1} \times L_{1}}, h_{K_{1}^{\prime} \times L_{1}^{\prime}}\right\rangle
$$

Note that since in this case $I \neq I^{\prime}$, we have that $X_{I} \cap X_{I^{\prime}}=\emptyset$, by (J1). Thus, $\mathbb{E}_{\theta} \theta_{K_{0}} \theta_{K_{0}^{\prime}} \theta_{K_{1}} \theta_{K_{1}^{\prime}} \neq 0$, only if $K_{0}=K_{1} \neq K_{0}^{\prime}=K_{1}^{\prime}$. Hence, in view of (R1)-(R4), we decompose the index set in (6.20) into the following four groups:

(a1) $K_{0}=K_{1} \neq K_{0}^{\prime}=K_{1}^{\prime}$ and $L_{0}=L_{1}=L_{0}^{\prime}=L_{1}^{\prime}$;

(a2) $K_{0}=K_{1} \neq K_{0}^{\prime}=K_{1}^{\prime}$ and $L_{0}=L_{1} \neq L_{0}^{\prime}=L_{1}^{\prime}$;

(a3) $K_{0}=K_{1} \neq K_{0}^{\prime}=K_{1}^{\prime}$ and $L_{0}=L_{1}^{\prime} \neq L_{0}^{\prime}=L_{1}$;

(a4) $K_{0}=K_{1} \neq K_{0}^{\prime}=K_{1}^{\prime}$ and $L_{0}=L_{0}^{\prime} \neq L_{1}=L_{1}^{\prime}$.

CASE 1, Group (A1): $K_{0}=K_{1} \neq K_{0}^{\prime}=K_{1}^{\prime}, L_{0}=L_{0}^{\prime}=L_{1}=L_{1}^{\prime}(((1)(0))((0)$ NIL) (NIL NIL)) - LEFT VARIANT. In this case, we have to estimate

$$
\sum_{K_{0}^{\prime}, L_{0}, K_{0}}\left\langle T h_{K_{0} \times L_{0}}, h_{K_{0}^{\prime} \times L_{0}}\right\rangle\left\langle T h_{K_{0} \times L_{0}}, h_{K_{0}^{\prime} \times L_{0}}\right\rangle .
$$

We put $a_{K_{0}, K_{0}^{\prime}, L_{0}}=\left\langle T h_{K_{0} \times L_{0}}, h_{K_{0}^{\prime} \times L_{0}}\right\rangle$ and note the estimate

$$
\left|a_{K_{0}, K_{0}^{\prime}, L_{0}}\right| \leq\|T\|\left|K_{0}\right|^{1 / p}\left|L_{0}\right|^{1 / q}\left|K_{0}^{\prime}\right|^{1 / p^{\prime}}\left|L_{0}\right|^{1 / q^{\prime}} .
$$

Now, we write (6.21) as follows:

$$
\sum_{K_{0}^{\prime}, L_{0}}\left\langle T \sum_{K_{0}} a_{K_{0}, K_{0}^{\prime}, L_{0}} h_{K_{0} \times L_{0}}, h_{K_{0}^{\prime} \times L_{0}}\right\rangle
$$

By duality, we obtain the subsequent upper estimate for (6.23):

$$
\sum_{K_{0}^{\prime}, L_{0}}\left\|T \sum_{K_{0}} a_{K_{0}, K_{0}^{\prime}, L_{0}} h_{K_{0} \times L_{0}}\right\|_{H^{p}\left(H^{q}\right)}\left\|h_{K_{0}^{\prime} \times L_{0}}\right\|_{\left(H^{p}\left(H^{q}\right)\right)^{*}} \cdot
$$

Estimate (6.22) and the disjointness of the dyadic intervals (see (J1)) yield

$$
\sum_{K_{0}^{\prime}, L_{0}}\|T\|\left\|\sum_{K_{0}} \max _{K_{0}}\left(\|T\|\left|K_{0}\right|^{1 / p}\left|L_{0}\right|^{1 / q}\left|K_{0}^{\prime}\right|^{1 / p^{\prime}}\left|L_{0}\right|^{1 / q^{\prime}}\right) h_{K_{0} \times L_{0}}\right\|_{H^{p}\left(H^{q}\right)}\left\|h_{K_{0}^{\prime} \times L_{0}}\right\|_{\left(H^{p}\left(H^{q}\right)\right)^{*}} \text {. }
$$

Consequently, we obtain

$$
\sum_{K_{0}^{\prime}, L_{0}}\|T\| \max _{K_{0}}\left(\|T\|\left|K_{0}\right|^{1 / p}\left|L_{0}\right|^{1 / q}\left|K_{0}^{\prime}\right|^{1 / p^{\prime}}\left|L_{0}\right|^{1 / q^{\prime}}\right)\left\|\sum_{K_{0}} h_{K_{0} \times L_{0}}\right\|_{H^{p}\left(H^{q}\right)}\left\|h_{K_{0}^{\prime} \times L_{0}}\right\|_{\left(H^{p}\left(H^{q}\right)\right)^{*}} .
$$


Thus, (6.26) is bounded from above by

$$
\|T\|^{2} \max _{K_{0}}\left|K_{0}\right|^{1 / p} \sum_{K_{0}^{\prime}, L_{0}}\left|L_{0}\right|^{2}\left|K_{0}^{\prime}\right|^{2 / p^{\prime}} .
$$

Using Hölder's inequality yields

$$
\|T\|^{2} \max _{K_{0}, K_{0}^{\prime}, L_{0}}\left|K_{0}\right|^{1 / p}\left|L_{0}\right|\left|K_{0}^{\prime}\right|^{2 / p^{\prime}-1} .
$$

Inserting $\left|K_{0}\right|,\left|L_{0}\right|,\left|K_{0}^{\prime}\right|,\left|L_{0}^{\prime}\right| \leq \alpha$ (see (4.15)) into (6.27), we obtain the estimate

$$
\|T\|^{2} \alpha^{1+1 / p^{\prime}}
$$

CASE 2, Group (A2): $K_{0}=K_{1} \neq K_{0}^{\prime}=K_{1}^{\prime}, L_{0}=L_{1} \neq L_{0}^{\prime}=L_{1}^{\prime}(((1)(1))((0)$ (0)) (NIL NIL)) - LEFT VARIANT. In this case, we have to estimate

$$
\sum_{K_{0}^{\prime}, L_{0}^{\prime}, K_{0}, L_{0}}\left\langle T h_{K_{0} \times L_{0}}, h_{K_{0}^{\prime} \times L_{0}^{\prime}}\right\rangle\left\langle T h_{K_{0} \times L_{0}}, h_{K_{0}^{\prime} \times L_{0}^{\prime}}\right\rangle .
$$

We put $a_{K_{0}, K_{0}^{\prime}, L_{0}, L_{0}^{\prime}}=\left\langle T h_{K_{0} \times L_{0}}, h_{K_{0}^{\prime} \times L_{0}^{\prime}}\right\rangle$ and note the estimate

$$
\left|a_{K_{0}, K_{0}^{\prime}, L_{0}, L_{0}^{\prime}}\right| \leq\|T\|\left|K_{0}\right|^{1 / p}\left|L_{0}\right|^{1 / q}\left|K_{0}^{\prime}\right|^{1 / p^{\prime}}\left|L_{0}^{\prime}\right|^{1 / q^{\prime}} .
$$

Now, we write (6.29) as follows:

$$
\sum_{K_{0}^{\prime}, L_{0}^{\prime}}\left\langle T \sum_{K_{0}, L_{0}} a_{K_{0}, K_{0}^{\prime}, L_{0}, L_{0}^{\prime}} h_{K_{0} \times L_{0}}, h_{K_{0}^{\prime} \times L_{0}^{\prime}}\right\rangle .
$$

By duality, we obtain the subsequent upper estimate for (6.31):

$$
\sum_{K_{0}^{\prime}, L_{0}^{\prime}}\left\|T \sum_{K_{0}, L_{0}} a_{K_{0}, K_{0}^{\prime}, L_{0}, L_{0}^{\prime}} h_{K_{0} \times L_{0}}\right\|_{H^{p}\left(H^{q}\right)}\left\|h_{K_{0}^{\prime} \times L_{0}^{\prime}}\right\|_{\left(H^{p}\left(H^{q}\right)\right)^{*}} .
$$

Estimate (6.30) and the disjointness of the dyadic intervals (see (J1) ) yield

$$
\sum_{K_{0}^{\prime}, L_{0}^{\prime}}\|T\|\left\|\sum_{K_{0}, L_{0}} \max _{K_{0}, L_{0}}\left(\|T\|\left|K_{0}\right|^{1 / p}\left|L_{0}\right|^{1 / q}\left|K_{0}^{\prime}\right|^{1 / p^{\prime}}\left|L_{0}^{\prime}\right|^{1 / q^{\prime}}\right) h_{K_{0} \times L_{0}}\right\|\left\|_{H^{p}\left(H^{q}\right)}\right\| h_{K_{0}^{\prime} \times L_{0}^{\prime}} \|_{\left(H^{p}\left(H^{q}\right)\right)^{*}} .
$$

Consequently, we obtain

$$
\sum_{K_{0}^{\prime}, L_{0}^{\prime}}\|T\| \max _{K_{0}, L_{0}}\left(\|T\|\left|K_{0}\right|^{1 / p}\left|L_{0}\right|^{1 / q}\left|K_{0}^{\prime}\right|^{1 / p^{\prime}}\left|L_{0}^{\prime}\right|^{1 / q^{\prime}}\right)\left\|\sum_{K_{0}, L_{0}} h_{K_{0} \times L_{0}}\right\|\left\|_{H^{p}\left(H^{q}\right)}\right\| h_{K_{0}^{\prime} \times L_{0}^{\prime}} \|_{\left(H^{p}\left(H^{q}\right)\right)^{*}} .
$$

Thus, 6.34) is bounded from above by

$$
\|T\|^{2} \max _{K_{0}, L_{0}}\left|K_{0}\right|^{1 / p}\left|L_{0}\right|^{1 / q} \sum_{K_{0}^{\prime}, L_{0}^{\prime}}\left|K_{0}^{\prime}\right|^{2 / p^{\prime}}\left|L_{0}^{\prime}\right|^{2 / q^{\prime}}
$$

Using Hölder's inequality yields

$$
\|T\|^{2} \max _{K_{0}, L_{0}, K_{0}^{\prime}, L_{0}^{\prime}}\left|K_{0}\right|^{1 / p}\left|L_{0}\right|^{1 / q}\left|K_{0}^{\prime}\right|^{2 / p^{\prime}-1}\left|L_{0}^{\prime}\right|^{2 / q^{\prime}-1} .
$$

Inserting $\left|K_{0}\right|,\left|L_{0}\right|,\left|K_{0}^{\prime}\right|,\left|L_{0}^{\prime}\right| \leq \alpha$ (see (4.15)) into (6.35), we obtain the estimate

$$
\|T\|^{2} \alpha^{1 / p^{\prime}+1 / q^{\prime}} \text {. }
$$


CASe 3, Group (A2): $K_{0}=K_{1} \neq K_{0}^{\prime}=K_{1}^{\prime}, L_{0}=L_{1} \neq L_{0}^{\prime}=L_{1}^{\prime}(((0)(0))(\mathrm{NIL}$ NIL) ((1) (1))) - RIGHT VARIANT. In this case, we have to estimate

$$
\sum_{K_{0}, L_{0}, K_{0}^{\prime}, L_{0}^{\prime}}\left\langle T h_{K_{0} \times L_{0}}, h_{K_{0}^{\prime} \times L_{0}^{\prime}}\right\rangle\left\langle T h_{K_{0} \times L_{0}}, h_{K_{0}^{\prime} \times L_{0}^{\prime}}\right\rangle .
$$

We put $a_{K_{0}, K_{0}^{\prime}, L_{0}, L_{0}^{\prime}}=\left\langle T h_{K_{0} \times L_{0}}, h_{K_{0}^{\prime} \times L_{0}^{\prime}}\right\rangle$ and note the estimate

$$
\left|a_{K_{0}, K_{0}^{\prime}, L_{0}, L_{0}^{\prime}}\right| \leq\|T\|\left|K_{0}\right|^{1 / p}\left|L_{0}\right|^{1 / q}\left|K_{0}^{\prime}\right|^{1 / p^{\prime}}\left|L_{0}^{\prime}\right|^{1 / q^{\prime}} .
$$

Now, we write (6.37) as follows:

$$
\sum_{K_{0}, L_{0}}\left\langle T h_{K_{0} \times L_{0}}, \sum_{K_{0}^{\prime}, L_{0}^{\prime}} a_{K_{0}, K_{0}^{\prime}, L_{0}, L_{0}^{\prime}} h_{K_{0}^{\prime} \times L_{0}^{\prime}}\right\rangle .
$$

By duality, we obtain the subsequent upper estimate for (6.39):

$$
\sum_{K_{0}, L_{0}}\left\|T h_{K_{0} \times L_{0}}\right\|_{H^{p}\left(H^{q}\right)}\left\|\sum_{K_{0}^{\prime}, L_{0}^{\prime}} a_{K_{0}, K_{0}^{\prime}, L_{0}, L_{0}^{\prime}} h_{K_{0}^{\prime} \times L_{0}^{\prime}}\right\|_{\left(H^{p}\left(H^{q}\right)\right)^{*}} .
$$

Estimate (6.38) and the disjointness of the dyadic intervals (see (J1)) yield

$$
\sum_{K_{0}, L_{0}}\|T\|\left\|h_{K_{0} \times L_{0}}\right\|_{H^{p}\left(H^{q}\right)}\left\|\sum_{K_{0}^{\prime}, L_{0}^{\prime}} \max _{K_{0}^{\prime}, L_{0}^{\prime}}\left(\|T\|\left|K_{0}\right|^{1 / p}\left|L_{0}\right|^{1 / q}\left|K_{0}^{\prime}\right|^{1 / p^{\prime}}\left|L_{0}^{\prime}\right|^{1 / q^{\prime}}\right) h_{K_{0}^{\prime} \times L_{0}^{\prime}}\right\|_{\left(H^{p}\left(H^{q}\right)\right)^{*}} .
$$

Consequently, we obtain

$$
\sum_{K_{0}, L_{0}}\|T\|\left\|h_{K_{0} \times L_{0}}\right\|_{H^{p}\left(H^{q}\right)} \max _{K_{0}^{\prime}, L_{0}^{\prime}}\left(\|T\|\left|K_{0}\right|^{1 / p}\left|L_{0}\right|^{1 / q}\left|K_{0}^{\prime}\right|^{1 / p^{\prime}}\left|L_{0}^{\prime}\right|^{1 / q^{\prime}}\right)\left\|\sum_{K_{0}^{\prime}, L_{0}^{\prime}} h_{K_{0}^{\prime} \times L_{0}^{\prime}}\right\|_{\left(H^{p}\left(H^{q}\right)\right)^{*}} .
$$

Thus, (6.42) is bounded from above by

$$
\|T\|^{2} \max _{K_{0}^{\prime}, L_{0}^{\prime}}\left|K_{0}^{\prime}\right|^{1 / p^{\prime}}\left|L_{0}^{\prime}\right|^{1 / q^{\prime}} \sum_{K_{0}, L_{0}}\left|K_{0}\right|^{2 / p}\left|L_{0}\right|^{2 / q} .
$$

Using Hölder's inequality yields

$$
\|T\|^{2} \max _{K_{0}, L_{0}, K_{0}^{\prime}, L_{0}^{\prime}}\left|K_{0}\right|^{2 / p-1}\left|L_{0}\right|^{2 / q-1}\left|K_{0}^{\prime}\right|^{1 / p^{\prime}}\left|L_{0}^{\prime}\right|^{1 / q^{\prime}} .
$$

Inserting $\left|K_{0}\right|,\left|L_{0}\right|,\left|K_{0}^{\prime}\right|,\left|L_{0}^{\prime}\right| \leq \alpha$ (see (4.15)) into (6.43), we obtain the estimate

$$
\|T\|^{2} \alpha^{1 / p+1 / q} .
$$

Summary of Case 2 and Case 3. Combining (6.36) with (6.44) yields

$$
\mathbb{E}_{\theta, \varepsilon} X^{2} \leq\|T\|^{2} \alpha .
$$

CASE 4, GRoup (A3): $K_{0}=K_{1} \neq K_{0}^{\prime}=K_{1}^{\prime}, L_{0}=L_{1}^{\prime} \neq L_{0}^{\prime}=L_{1}(((1)(0))((0)$ (1)) (NIL NIL)) - LEFT VARIANT. In this case, we have to estimate

$$
\sum_{K_{0}^{\prime}, L_{0}, K_{0}, L_{0}^{\prime}}\left\langle T h_{K_{0} \times L_{0}}, h_{K_{0}^{\prime} \times L_{0}^{\prime}}\right\rangle\left\langle T h_{K_{0} \times L_{0}^{\prime}}, h_{K_{0}^{\prime} \times L_{0}}\right\rangle .
$$

We put $a_{K_{0}, K_{0}^{\prime}, L_{0}, L_{0}^{\prime}}=\left\langle T h_{K_{0} \times L_{0}}, h_{K_{0}^{\prime} \times L_{0}^{\prime}}\right\rangle$ and note the estimate

$$
\left|a_{K_{0}, K_{0}^{\prime}, L_{0}, L_{0}^{\prime}}\right| \leq\|T\|\left|K_{0}\right|^{1 / p}\left|L_{0}\right|^{1 / q}\left|K_{0}^{\prime}\right|^{1 / p^{\prime}}\left|L_{0}^{\prime}\right|^{1 / q^{\prime}} .
$$

Now, we write (6.46) as follows:

$$
\sum_{K_{0}^{\prime}, L_{0}}\left\langle T \sum_{K_{0}, L_{0}^{\prime}} a_{K_{0}, K_{0}^{\prime}, L_{0}, L_{0}^{\prime}} h_{K_{0} \times L_{0}^{\prime}}, h_{K_{0}^{\prime} \times L_{0}}\right\rangle .
$$


By duality, we obtain the subsequent upper estimate for 6.48):

$$
\sum_{K_{0}^{\prime}, L_{0}}\left\|T \sum_{K_{0}, L_{0}^{\prime}} a_{K_{0}, K_{0}^{\prime}, L_{0}, L_{0}^{\prime}} h_{K_{0} \times L_{0}^{\prime}}\right\|_{H^{p}\left(H^{q}\right)}\left\|h_{K_{0}^{\prime} \times L_{0}}\right\|_{\left(H^{p}\left(H^{q}\right)\right)^{*}} .
$$

Estimate (6.47) and the disjointness of the dyadic intervals (see (J1) ) yield

$$
\sum_{K_{0}^{\prime}, L_{0}}\|T\|\left\|\sum_{K_{0}, L_{0}^{\prime}} \max _{K_{0}, L_{0}^{\prime}}\left(\|T\|\left|K_{0}\right|^{1 / p}\left|L_{0}\right|^{1 / q}\left|K_{0}^{\prime}\right|^{1 / p^{\prime}}\left|L_{0}^{\prime}\right|^{1 / q^{\prime}}\right) h_{K_{0} \times L_{0}^{\prime}}\right\|_{H^{p}\left(H^{q}\right)}\left\|h_{K_{0}^{\prime} \times L_{0}}\right\|_{\left(H^{p}\left(H^{q}\right)\right)^{*}} .
$$

Consequently, we obtain

$$
\sum_{K_{0}^{\prime}, L_{0}}\|T\| \max _{K_{0}, L_{0}^{\prime}}\left(\|T\|\left|K_{0}\right|^{1 / p}\left|L_{0}\right|^{1 / q}\left|K_{0}^{\prime}\right|^{1 / p^{\prime}}\left|L_{0}^{\prime}\right|^{1 / q^{\prime}}\right)\left\|\sum_{K_{0}, L_{0}^{\prime}} h_{K_{0} \times L_{0}^{\prime}}\right\|_{H^{p}\left(H^{q}\right)}\left\|h_{K_{0}^{\prime} \times L_{0}}\right\|_{\left(H^{p}\left(H^{q}\right)\right)^{*}} .
$$

Thus, 6.51) is bounded from above by

$$
\|T\|^{2} \max _{K_{0}, L_{0}^{\prime}}\left|K_{0}\right|^{1 / p}\left|L_{0}^{\prime}\right|^{1 / q^{\prime}} \sum_{K_{0}^{\prime}, L_{0}}\left|K_{0}^{\prime}\right|^{2 / p^{\prime}}\left|L_{0}\right| .
$$

Using Hölder's inequality yields

$$
\|T\|^{2} \max _{K_{0}, K_{0}^{\prime}, L_{0}, L_{0}^{\prime}}\left|K_{0}\right|^{1 / p}\left|K_{0}^{\prime}\right|^{2 / p^{\prime}-1}\left|L_{0}^{\prime}\right|^{1 / q^{\prime}}
$$

Inserting $\left|K_{0}\right|,\left|K_{0}^{\prime}\right|,\left|L_{0}^{\prime}\right| \leq \alpha$ (see (4.15) ) into (6.52), we obtain the estimate

$$
\|T\|^{2} \alpha^{1 / p^{\prime}+1 / q^{\prime}} \text {. }
$$

CASE 5, Group (A3) : $K_{0}=K_{1} \neq K_{0}^{\prime}=K_{1}^{\prime}, L_{0}=L_{1}^{\prime} \neq L_{0}^{\prime}=L_{1}(((0)(1))$ (NIL NIL) $((1)(0)))$ - RIGHT VARIANT. In this case, we have to estimate

$$
\sum_{K_{0}, L_{0}^{\prime}, K_{0}^{\prime}, L_{0}}\left\langle T h_{K_{0} \times L_{0}}, h_{K_{0}^{\prime} \times L_{0}^{\prime}}\right\rangle\left\langle T h_{K_{0} \times L_{0}^{\prime}}, h_{K_{0}^{\prime} \times L_{0}}\right\rangle .
$$

We put $a_{K_{0}, K_{0}^{\prime}, L_{0}, L_{0}^{\prime}}=\left\langle T h_{K_{0} \times L_{0}}, h_{K_{0}^{\prime} \times L_{0}^{\prime}}\right\rangle$ and note the estimate

$$
\left|a_{K_{0}, K_{0}^{\prime}, L_{0}, L_{0}^{\prime}}\right| \leq\|T\|\left|K_{0}\right|^{1 / p}\left|L_{0}\right|^{1 / q}\left|K_{0}^{\prime}\right|^{1 / p^{\prime}}\left|L_{0}^{\prime}\right|^{1 / q^{\prime}} .
$$

Now, we write (6.54) as follows:

$$
\sum_{K_{0}, L_{0}^{\prime}}\left\langle T h_{K_{0} \times L_{0}^{\prime}}, \sum_{K_{0}^{\prime}, L_{0}} a_{K_{0}, K_{0}^{\prime}, L_{0}, L_{0}^{\prime}} h_{K_{0}^{\prime} \times L_{0}}\right\rangle .
$$

By duality, we obtain the subsequent upper estimate for 6.56):

$$
\sum_{K_{0}, L_{0}^{\prime}}\left\|T h_{K_{0} \times L_{0}^{\prime}}\right\|_{H^{p}\left(H^{q}\right)}\left\|\sum_{K_{0}^{\prime}, L_{0}} a_{K_{0}, K_{0}^{\prime}, L_{0}, L_{0}^{\prime}} h_{K_{0}^{\prime} \times L_{0}}\right\|_{\left(H^{p}\left(H^{q}\right)\right)^{*}} .
$$

Estimate (6.55) and the disjointness of the dyadic intervals (see (J1) ) yield

$$
\sum_{K_{0}, L_{0}^{\prime}}\|T\|\left\|h_{K_{0} \times L_{0}^{\prime}}\right\|_{H^{p}\left(H^{q}\right)}\left\|\sum_{K_{0}^{\prime}, L_{0}} \max _{K_{0}^{\prime}, L_{0}}\left(\|T\|\left|K_{0}\right|^{1 / p}\left|L_{0}\right|^{1 / q}\left|K_{0}^{\prime}\right|^{1 / p^{\prime}}\left|L_{0}^{\prime}\right|^{1 / q^{\prime}}\right) h_{K_{0}^{\prime} \times L_{0}}\right\|_{\left(H^{p}\left(H^{q}\right)\right)^{*}} .
$$


Consequently, we obtain

$$
\sum_{K_{0}, L_{0}^{\prime}}\|T\|\left\|h_{K_{0} \times L_{0}^{\prime}}\right\|_{H^{p}\left(H^{q}\right)} \max _{K_{0}^{\prime}, L_{0}}\left(\|T\|\left|K_{0}\right|^{1 / p}\left|L_{0}\right|^{1 / q}\left|K_{0}^{\prime}\right|^{1 / p^{\prime}}\left|L_{0}^{\prime}\right|^{1 / q^{\prime}}\right)\left\|\sum_{K_{0}^{\prime}, L_{0}} h_{K_{0}^{\prime} \times L_{0}}\right\|_{\left(H^{p}\left(H^{q}\right)\right)^{*}} .
$$

Thus, (6.59) is bounded from above by

$$
\|T\|^{2} \max _{K_{0}^{\prime}, L_{0}}\left|L_{0}\right|^{1 / q}\left|K_{0}^{\prime}\right|^{1 / p^{\prime}} \sum_{K_{0}, L_{0}^{\prime}}\left|K_{0}\right|^{2 / p}\left|L_{0}^{\prime}\right| .
$$

Using Hölder's inequality yields

$$
\|T\|^{2} \max _{K_{0}, K_{0}^{\prime}, L_{0}}\left|K_{0}\right|^{2 / p-1}\left|K_{0}^{\prime}\right|^{1 / p^{\prime}}\left|L_{0}\right|^{1 / q} .
$$

Inserting $\left|K_{0}\right|,\left|K_{0}^{\prime}\right|,\left|L_{0}\right| \leq \alpha$ (see (4.15) ) into (6.60), we obtain the estimate

$$
\|T\|^{2} \alpha^{1 / p+1 / q} \text {. }
$$

Summary of Case 4 and Case 5. Combining (6.53) with (6.61) yields

$$
\mathbb{E}_{\theta, \varepsilon} X^{2} \leq\|T\|^{2} \alpha
$$

CAse 6, Group (A4) : $K_{0}=K_{1} \neq K_{0}^{\prime}=K_{1}^{\prime}, L_{0}=L_{0}^{\prime} \neq L_{1}=L_{1}^{\prime}(((1)(2))((0)$ (0)) (NIL NIL)) - LEFT VARIANT. In this case, we have to estimate

$$
\sum_{K_{0}^{\prime}, L_{1}, K_{0}, L_{0}}\left\langle T h_{K_{0} \times L_{0}}, h_{K_{0}^{\prime} \times L_{0}}\right\rangle\left\langle T h_{K_{0} \times L_{1}}, h_{K_{0}^{\prime} \times L_{1}}\right\rangle .
$$

We put $a_{K_{0}, K_{0}^{\prime}, L_{0}}=\left\langle T h_{K_{0} \times L_{0}}, h_{K_{0}^{\prime} \times L_{0}}\right\rangle$ and note the estimate

$$
\left|a_{K_{0}, K_{0}^{\prime}, L_{0}}\right| \leq\|T\|\left|K_{0}\right|^{1 / p}\left|L_{0}\right|^{1 / q}\left|K_{0}^{\prime}\right|^{1 / p^{\prime}}\left|L_{0}\right|^{1 / q^{\prime}} .
$$

Now, we write (6.63) as follows:

$$
\sum_{K_{0}^{\prime}, L_{1}}\left\langle T \sum_{K_{0}, L_{0}} a_{K_{0}, K_{0}^{\prime}, L_{0}} h_{K_{0} \times L_{1}}, h_{K_{0}^{\prime} \times L_{1}}\right\rangle .
$$

By duality, we obtain the subsequent upper estimate for (6.65):

$$
\sum_{K_{0}^{\prime}, L_{1}}\left\|T \sum_{K_{0}, L_{0}} a_{K_{0}, K_{0}^{\prime}, L_{0}} h_{K_{0} \times L_{1}}\right\|_{H^{p}\left(H^{q}\right)}\left\|h_{K_{0}^{\prime} \times L_{1}}\right\|_{\left(H^{p}\left(H^{q}\right)\right)^{*}} \cdot
$$

Estimate (6.64) and the disjointness of the dyadic intervals (see (J1)) yield

$$
\sum_{K_{0}^{\prime}, L_{1}}\|T\|\left\|\sum_{K_{0}, L_{0}} \max _{K_{0}}\left(\|T\|\left|K_{0}\right|^{1 / p}\left|L_{0}\right|^{1 / q}\left|K_{0}^{\prime}\right|^{1 / p^{\prime}}\left|L_{0}\right|^{1 / q^{\prime}}\right) h_{K_{0} \times L_{1}}\right\|_{H^{p}\left(H^{q}\right)}\left\|h_{K_{0}^{\prime} \times L_{1}}\right\|_{\left(H^{p}\left(H^{q}\right)\right)^{*}} \cdot
$$

Consequently, we obtain

$$
\sum_{K_{0}^{\prime}, L_{1}}\|T\| \sum_{L_{0}} \max _{K_{0}}\left(\|T\|\left|K_{0}\right|^{1 / p}\left|L_{0}\right|^{1 / q}\left|K_{0}^{\prime}\right|^{1 / p^{\prime}}\left|L_{0}\right|^{1 / q^{\prime}}\right)\left\|\sum_{K_{0}} h_{K_{0} \times L_{1}}\right\|_{H^{p}\left(H^{q}\right)}\left\|h_{K_{0}^{\prime} \times L_{1}}\right\|_{\left(H^{p}\left(H^{q}\right)\right)^{*}} .
$$

Thus, (6.68) is bounded from above by

$$
\|T\|^{2} \max _{K_{0}}\left|K_{0}\right|^{1 / p} \sum_{K_{0}^{\prime}, L_{1}} \sum_{L_{0}}\left|L_{0}\right|\left|K_{0}^{\prime}\right|^{2 / p^{\prime}}\left|L_{1}\right|
$$


Using Hölder's inequality yields

$$
\|T\|^{2} \max _{K_{0}, K_{0}^{\prime}}\left|K_{0}\right|^{1 / p}\left|K_{0}^{\prime}\right|^{2 / p^{\prime}-1}
$$

Inserting $\left|K_{0}\right|,\left|K_{0}^{\prime}\right| \leq \alpha$ (see (4.15) ) into (6.69), we obtain the estimate

$$
\|T\|^{2} \alpha^{1 / p^{\prime}}
$$

CASe 7, Group (A4) $: K_{0}=K_{1} \neq K_{0}^{\prime}=K_{1}^{\prime}, L_{0}=L_{0}^{\prime} \neq L_{1}=L_{1}^{\prime}(((0)(2))(\mathrm{NIL}$ NIL) $((1)(0)))$ - RIGHT VARIANT. In this case, we have to estimate

$$
\sum_{K_{0}, L_{1}, K_{0}^{\prime}, L_{0}}\left\langle T h_{K_{0} \times L_{0}}, h_{K_{0}^{\prime} \times L_{0}}\right\rangle\left\langle T h_{K_{0} \times L_{1}}, h_{K_{0}^{\prime} \times L_{1}}\right\rangle .
$$

We put $a_{K_{0}, K_{0}^{\prime}, L_{0}}=\left\langle T h_{K_{0} \times L_{0}}, h_{K_{0}^{\prime} \times L_{0}}\right\rangle$ and note the estimate

$$
\left|a_{K_{0}, K_{0}^{\prime}, L_{0}}\right| \leq\|T\|\left|K_{0}\right|^{1 / p}\left|L_{0}\right|^{1 / q}\left|K_{0}^{\prime}\right|^{1 / p^{\prime}}\left|L_{0}\right|^{1 / q^{\prime}} .
$$

Now, we write (6.71) as follows:

$$
\sum_{K_{0}, L_{1}}\left\langle T h_{K_{0} \times L_{1}}, \sum_{K_{0}^{\prime}, L_{0}} a_{K_{0}, K_{0}^{\prime}, L_{0}} h_{K_{0}^{\prime} \times L_{1}}\right\rangle .
$$

By duality, we obtain the subsequent upper estimate for (6.73):

$$
\sum_{K_{0}, L_{1}}\left\|T h_{K_{0} \times L_{1}}\right\|_{H^{p}\left(H^{q}\right)}\left\|\sum_{K_{0}^{\prime}, L_{0}} a_{K_{0}, K_{0}^{\prime}, L_{0}} h_{K_{0}^{\prime} \times L_{1}}\right\|_{\left(H^{p}\left(H^{q}\right)\right)^{*}} .
$$

Estimate (6.72) and the disjointness of the dyadic intervals (see (JI)) yield

$$
\sum_{K_{0}, L_{1}}\|T\|\left\|h_{K_{0} \times L_{1}}\right\|_{H^{p}\left(H^{q}\right)}\left\|\sum_{K_{0}^{\prime}, L_{0}} \max _{K_{0}^{\prime}}\left(\|T\|\left|K_{0}\right|^{1 / p}\left|L_{0}\right|^{1 / q}\left|K_{0}^{\prime}\right|^{1 / p^{\prime}}\left|L_{0}\right|^{1 / q^{\prime}}\right) h_{K_{0}^{\prime} \times L_{1}}\right\|_{\left(H^{p}\left(H^{q}\right)\right)^{*}} .
$$

Consequently, we obtain

$$
\sum_{K_{0}, L_{1}}\|T\|\left\|h_{K_{0} \times L_{1}}\right\|_{H^{p}\left(H^{q}\right)} \sum_{L_{0}} \max _{K_{0}^{\prime}}\left(\|T\|\left|K_{0}\right|^{1 / p}\left|L_{0}\right|^{1 / q}\left|K_{0}^{\prime}\right|^{1 / p^{\prime}}\left|L_{0}\right|^{1 / q^{\prime}}\right)\left\|\sum_{K_{0}^{\prime}} h_{K_{0}^{\prime} \times L_{1}}\right\|_{\left(H^{p}\left(H^{q}\right)\right)^{*}} .
$$

Thus, (6.76) is bounded from above by

$$
\|T\|^{2} \max _{K_{0}^{\prime}}\left|K_{0}^{\prime}\right|^{1 / p^{\prime}} \sum_{K_{0}, L_{1}} \sum_{L_{0}}\left|K_{0}\right|^{2 / p}\left|L_{0}\right|\left|L_{1}\right| .
$$

Using Hölder's inequality yields

$$
\|T\|^{2} \max _{K_{0}, K_{0}^{\prime}}\left|K_{0}\right|^{2 / p-1}\left|K_{0}^{\prime}\right|^{1 / p^{\prime}} .
$$

Inserting $\left|K_{0}\right|,\left|K_{0}^{\prime}\right| \leq \alpha$ (see (4.15) ) into (6.77), we obtain the estimate

$$
\|T\|^{2} \alpha^{1 / p} \text {. }
$$

Summary of Case 6 and Case 7. Combining (6.70) with (6.78) yields

$$
\mathbb{E}_{\theta, \varepsilon} X^{2} \leq\|T\|^{2} \alpha^{1 / 2} .
$$

Summary for $X$. Combining (6.28) with (6.45), (6.62) and (6.79) yields

$$
\mathbb{E}_{\theta, \varepsilon} X^{2} \leq 4\|T\|^{2} \alpha^{1 / 2}
$$


6.3. Estimates for $Y$. In this case, the following variables will always be summed over the following sets:

$$
\begin{aligned}
& \triangleright K_{0}, K_{1}, K_{0}^{\prime}, K_{1}^{\prime} \text { over } x_{I} ; \\
& \triangleright L_{0}, L_{1} \text { over } y_{J} ; \\
& \triangleright L_{0}^{\prime}, L_{1}^{\prime} \text { over } y_{J^{\prime}} .
\end{aligned}
$$

Proof. Note that by (4.14c) and (4.13) we obtain $Y^{2}(\theta, \varepsilon)$ is given by

$$
\sum_{\substack{K_{0}, K_{1}, K_{0}^{\prime}, K_{1}^{\prime} \\ L_{0}, L_{1}, L_{0}^{\prime}, L_{1}^{\prime}}} \theta_{K_{0}} \theta_{K_{1}} \theta_{K_{0}^{\prime}} \theta_{K_{1}^{\prime}} \varepsilon_{L_{0}} \varepsilon_{L_{1}} \varepsilon_{L_{0}^{\prime}} \varepsilon_{L_{1}^{\prime}}\left\langle T h_{K_{0} \times L_{0}}, h_{K_{0}^{\prime} \times L_{0}^{\prime}}\right\rangle\left\langle T h_{K_{1} \times L_{1}}, h_{K_{1}^{\prime} \times L_{1}^{\prime}}\right\rangle
$$

Note that since in this case $J \neq J^{\prime}$, we have that $y_{J} \cap y_{J^{\prime}}=\emptyset$, by (J1). Thus, $\mathbb{E}_{\varepsilon} \varepsilon_{L_{0}} \varepsilon_{L_{0}^{\prime}} \varepsilon_{L_{1}} \varepsilon_{L_{1}^{\prime}} \neq 0$, only if $L_{0}=L_{1} \neq L_{0}^{\prime}=L_{1}^{\prime}$. Hence, in view of (R1)-(R4), we decompose the index set in (6.81) into the following four groups:

(b1) $K_{0}=K_{1}=K_{0}^{\prime}=K_{1}^{\prime}$ and $L_{0}=L_{1} \neq L_{0}^{\prime}=L_{1}^{\prime}$;

(b2) $K_{0}=K_{1} \neq K_{0}^{\prime}=K_{1}^{\prime}$ and $L_{0}=L_{1} \neq L_{0}^{\prime}=L_{1}^{\prime}$;

(b3) $K_{0}=K_{1}^{\prime} \neq K_{0}^{\prime}=K_{1}$ and $L_{0}=L_{1} \neq L_{0}^{\prime}=L_{1}^{\prime}$;

(b4) $K_{0}=K_{0}^{\prime} \neq K_{1}=K_{1}^{\prime}$ and $L_{0}=L_{1} \neq L_{0}^{\prime}=L_{1}^{\prime}$.

Case 1, Group (B1) : $K_{0}=K_{0}^{\prime}=K_{1}=K_{1}^{\prime}, L_{0}=L_{1} \neq L_{0}^{\prime}=L_{1}^{\prime}(((0)(1))$ ( NIL (0)) (NIL NIL)) - LEFT VARIANT. In this case, we have to estimate

$$
\sum_{K_{0}, L_{0}^{\prime}, L_{0}}\left\langle T h_{K_{0} \times L_{0}}, h_{K_{0} \times L_{0}^{\prime}}\right\rangle\left\langle T h_{K_{0} \times L_{0}}, h_{K_{0} \times L_{0}^{\prime}}\right\rangle .
$$

We put $a_{K_{0}, L_{0}, L_{0}^{\prime}}=\left\langle T h_{K_{0} \times L_{0}}, h_{K_{0} \times L_{0}^{\prime}}\right\rangle$ and note the estimate

$$
\left|a_{K_{0}, L_{0}, L_{0}^{\prime}}\right| \leq\|T\|\left|K_{0}\right|^{1 / p}\left|L_{0}\right|^{1 / q}\left|K_{0}\right|^{1 / p^{\prime}}\left|L_{0}^{\prime}\right|^{1 / q^{\prime}} .
$$

Now, we write (6.82) as follows:

$$
\sum_{K_{0}, L_{0}^{\prime}}\left\langle T \sum_{L_{0}} a_{K_{0}, L_{0}, L_{0}^{\prime}} h_{K_{0} \times L_{0}}, h_{K_{0} \times L_{0}^{\prime}}\right\rangle .
$$

By duality, we obtain the subsequent upper estimate for (6.84):

$$
\sum_{K_{0}, L_{0}^{\prime}}\left\|T \sum_{L_{0}} a_{K_{0}, L_{0}, L_{0}^{\prime}} h_{K_{0} \times L_{0}}\right\|_{H^{p}\left(H^{q}\right)}\left\|h_{K_{0} \times L_{0}^{\prime}}\right\|_{\left(H^{p}\left(H^{q}\right)\right)^{*}} \cdot
$$

Estimate (6.83) and the disjointness of the dyadic intervals (see (J1) yield

$$
\sum_{K_{0}, L_{0}^{\prime}}\|T\|\left\|\sum_{L_{0}} \max _{L_{0}}\left(\|T\|\left|K_{0}\right|^{1 / p}\left|L_{0}\right|^{1 / q}\left|K_{0}\right|^{1 / p^{\prime}}\left|L_{0}^{\prime}\right|^{1 / q^{\prime}}\right) h_{K_{0} \times L_{0}}\right\|_{H^{p}\left(H^{q}\right)}\left\|h_{K_{0} \times L_{0}^{\prime}}\right\|_{\left(H^{p}\left(H^{q}\right)\right)^{*}} .
$$

Consequently, we obtain

$$
\sum_{K_{0}, L_{0}^{\prime}}\|T\| \max _{L_{0}}\left(\|T\|\left|K_{0}\right|^{1 / p}\left|L_{0}\right|^{1 / q}\left|K_{0}\right|^{1 / p^{\prime}}\left|L_{0}^{\prime}\right|^{1 / q^{\prime}}\right)\left\|\sum_{L_{0}} h_{K_{0} \times L_{0}}\right\|_{H^{p}\left(H^{q}\right)}\left\|h_{K_{0} \times L_{0}^{\prime}}\right\|_{\left(H^{p}\left(H^{q}\right)\right)^{*}} .
$$

Thus, 6.87) is bounded from above by

$$
\|T\|^{2} \max _{L_{0}}\left|L_{0}\right|^{1 / q} \sum_{K_{0}, L_{0}^{\prime}}\left|K_{0}\right|^{2}\left|L_{0}^{\prime}\right|^{2 / q^{\prime}} .
$$

Using Hölder's inequality yields

$$
\|T\|^{2} \max _{K_{0}, L_{0}, L_{0}^{\prime}}\left|K_{0}\right|\left|L_{0}\right|^{1 / q}\left|L_{0}^{\prime}\right|^{2 / q^{\prime}-1} .
$$


Inserting $\left|K_{0}\right|,\left|L_{0}\right|,\left|L_{0}^{\prime}\right| \leq \alpha$ (see (4.15) ) into (6.88), we obtain the estimate

$$
\|T\|^{2} \alpha^{1+1 / q^{\prime}} \text {. }
$$

Case 2, Group (B2) : $K_{0}=K_{1} \neq K_{0}^{\prime}=K_{1}^{\prime}, L_{0}=L_{1} \neq L_{0}^{\prime}=L_{1}^{\prime}(((1)(1))((0)$ (0)) (NIL NIL)) - LEFT VARIANT. In this case, we have to estimate

$$
\sum_{K_{0}^{\prime}, L_{0}^{\prime}, K_{0}, L_{0}}\left\langle T h_{K_{0} \times L_{0}}, h_{K_{0}^{\prime} \times L_{0}^{\prime}}\right\rangle\left\langle T h_{K_{0} \times L_{0}}, h_{K_{0}^{\prime} \times L_{0}^{\prime}}\right\rangle .
$$

We put $a_{K_{0}, K_{0}^{\prime}, L_{0}, L_{0}^{\prime}}=\left\langle T h_{K_{0} \times L_{0}}, h_{K_{0}^{\prime} \times L_{0}^{\prime}}\right\rangle$ and note the estimate

$$
\left|a_{K_{0}, K_{0}^{\prime}, L_{0}, L_{0}^{\prime}}\right| \leq\|T\|\left|K_{0}\right|^{1 / p}\left|L_{0}\right|^{1 / q}\left|K_{0}^{\prime}\right|^{1 / p^{\prime}}\left|L_{0}^{\prime}\right|^{1 / q^{\prime}} .
$$

Now, we write (6.90) as follows:

$$
\sum_{K_{0}^{\prime}, L_{0}^{\prime}}\left\langle T \sum_{K_{0}, L_{0}} a_{K_{0}, K_{0}^{\prime}, L_{0}, L_{0}^{\prime}} h_{K_{0} \times L_{0}}, h_{K_{0}^{\prime} \times L_{0}^{\prime}}\right\rangle .
$$

By duality, we obtain the subsequent upper estimate for (6.92):

$$
\sum_{K_{0}^{\prime}, L_{0}^{\prime}}\left\|T \sum_{K_{0}, L_{0}} a_{K_{0}, K_{0}^{\prime}, L_{0}, L_{0}^{\prime}} h_{K_{0} \times L_{0}}\right\|_{H^{p}\left(H^{q}\right)}\left\|h_{K_{0}^{\prime} \times L_{0}^{\prime}}\right\|_{\left(H^{p}\left(H^{q}\right)\right)^{*}} .
$$

Estimate (6.91) and the disjointness of the dyadic intervals (see (J1) yield

$$
\sum_{K_{0}^{\prime}, L_{0}^{\prime}}\|T\|\left\|\sum_{K_{0}, L_{0}} \max _{K_{0}, L_{0}}\left(\|T\|\left|K_{0}\right|^{1 / p}\left|L_{0}\right|^{1 / q}\left|K_{0}^{\prime}\right|^{1 / p^{\prime}}\left|L_{0}^{\prime}\right|^{1 / q^{\prime}}\right) h_{K_{0} \times L_{0}}\right\|\left\|_{H^{p}\left(H^{q}\right)}\right\| h_{K_{0}^{\prime} \times L_{0}^{\prime}} \|_{\left(H^{p}\left(H^{q}\right)\right)^{*}} \text {. }
$$

Consequently, we obtain

$$
\sum_{K_{0}^{\prime}, L_{0}^{\prime}}\|T\| \max _{K_{0}, L_{0}}\left(\|T\|\left|K_{0}\right|^{1 / p}\left|L_{0}\right|^{1 / q}\left|K_{0}^{\prime}\right|^{1 / p^{\prime}}\left|L_{0}^{\prime}\right|^{1 / q^{\prime}}\right)\left\|\sum_{K_{0}, L_{0}} h_{K_{0} \times L_{0}}\right\|_{H^{p}\left(H^{q}\right)}\left\|h_{K_{0}^{\prime} \times L_{0}^{\prime}}\right\|_{\left(H^{p}\left(H^{q}\right)\right)^{*}} .
$$

Thus, 6.95) is bounded from above by

$$
\|T\|^{2} \max _{K_{0}, L_{0}}\left|K_{0}\right|^{1 / p}\left|L_{0}\right|^{1 / q} \sum_{K_{0}^{\prime}, L_{0}^{\prime}}\left|K_{0}^{\prime}\right|^{2 / p^{\prime}}\left|L_{0}^{\prime}\right|^{2 / q^{\prime}} .
$$

Using Hölder's inequality yields

$$
\|T\|^{2} \max _{K_{0}, L_{0}, K_{0}^{\prime}, L_{0}^{\prime}}\left|K_{0}\right|^{1 / p}\left|L_{0}\right|^{1 / q}\left|K_{0}^{\prime}\right|^{2 / p^{\prime}-1}\left|L_{0}^{\prime}\right|^{2 / q^{\prime}-1} .
$$

Inserting $\left|K_{0}\right|,\left|K_{0}^{\prime}\right|,\left|L_{0}\right|,\left|L_{0}^{\prime}\right| \leq \alpha$ (see (4.15)) into (6.96), we obtain the estimate

$$
\|T\|^{2} \alpha^{1 / p^{\prime}+1 / q^{\prime}} \text {. }
$$

Case 3, Group (B2) $: K_{0}=K_{1} \neq K_{0}^{\prime}=K_{1}^{\prime}, L_{0}=L_{1} \neq L_{0}^{\prime}=L_{1}^{\prime}(((0)(0))(\mathrm{NIL}$ NIL) ((1) (1))) - RIGHT VARIANT. In this case, we have to estimate

$$
\sum_{K_{0}, L_{0}, K_{0}^{\prime}, L_{0}^{\prime}}\left\langle T h_{K_{0} \times L_{0}}, h_{K_{0}^{\prime} \times L_{0}^{\prime}}\right\rangle\left\langle T h_{K_{0} \times L_{0}}, h_{K_{0}^{\prime} \times L_{0}^{\prime}}\right\rangle .
$$

We put $a_{K_{0}, K_{0}^{\prime}, L_{0}, L_{0}^{\prime}}=\left\langle T h_{K_{0} \times L_{0}}, h_{K_{0}^{\prime} \times L_{0}^{\prime}}\right\rangle$ and note the estimate

$$
\left|a_{K_{0}, K_{0}^{\prime}, L_{0}, L_{0}^{\prime}}\right| \leq\|T\|\left|K_{0}\right|^{1 / p}\left|L_{0}\right|^{1 / q}\left|K_{0}^{\prime}\right|^{1 / p^{\prime}}\left|L_{0}^{\prime}\right|^{1 / q^{\prime}} .
$$


Now, we write (6.98) as follows:

$$
\sum_{K_{0}, L_{0}}\left\langle T h_{K_{0} \times L_{0}}, \sum_{K_{0}^{\prime}, L_{0}^{\prime}} a_{K_{0}, K_{0}^{\prime}, L_{0}, L_{0}^{\prime}} h_{K_{0}^{\prime} \times L_{0}^{\prime}}\right\rangle .
$$

By duality, we obtain the subsequent upper estimate for (6.100):

$$
\sum_{K_{0}, L_{0}}\left\|T h_{K_{0} \times L_{0}}\right\|_{H^{p}\left(H^{q}\right)}\left\|\sum_{K_{0}^{\prime}, L_{0}^{\prime}} a_{K_{0}, K_{0}^{\prime}, L_{0}, L_{0}^{\prime}} h_{K_{0}^{\prime} \times L_{0}^{\prime}}\right\|_{\left(H^{p}\left(H^{q}\right)\right)^{*}} .
$$

Estimate (6.99) and the disjointness of the dyadic intervals (see (J1)) yield

$$
\sum_{K_{0}, L_{0}}\|T\|\left\|h_{K_{0} \times L_{0}}\right\|_{H^{p}\left(H^{q}\right)}\left\|\sum_{K_{0}^{\prime}, L_{0}^{\prime}} \max _{K_{0}^{\prime}, L_{0}^{\prime}}\left(\|T\|\left|K_{0}\right|^{1 / p}\left|L_{0}\right|^{1 / q}\left|K_{0}^{\prime}\right|^{1 / p^{\prime}}\left|L_{0}^{\prime}\right|^{1 / q^{\prime}}\right) h_{K_{0}^{\prime} \times L_{0}^{\prime}}\right\|_{\left(H^{p}\left(H^{q}\right)\right)^{*}} \text {. }
$$

Consequently, we obtain

$$
\sum_{K_{0}, L_{0}}\|T\|\left\|h_{K_{0} \times L_{0}}\right\|_{H^{p}\left(H^{q}\right)} \max _{K_{0}^{\prime}, L_{0}^{\prime}}\left(\|T\|\left|K_{0}\right|^{1 / p}\left|L_{0}\right|^{1 / q}\left|K_{0}^{\prime}\right|^{1 / p^{\prime}}\left|L_{0}^{\prime}\right|^{1 / q^{\prime}}\right)\left\|\sum_{K_{0}^{\prime}, L_{0}^{\prime}} h_{K_{0}^{\prime} \times L_{0}^{\prime}}\right\|_{\left(H^{p}\left(H^{q}\right)\right)^{*}} .
$$

Thus, 6.103) is bounded from above by

$$
\|T\|^{2} \max _{K_{0}^{\prime}, L_{0}^{\prime}}\left|K_{0}^{\prime}\right|^{1 / p^{\prime}}\left|L_{0}^{\prime}\right|^{1 / q^{\prime}} \sum_{K_{0}, L_{0}}\left|K_{0}\right|^{2 / p}\left|L_{0}\right|^{2 / q} .
$$

Using Hölder's inequality yields

$$
\|T\|^{2} \max _{K_{0}, K_{0}^{\prime}, L_{0}, L_{0}^{\prime}}\left|K_{0}\right|^{2 / p-1}\left|L_{0}\right|^{2 / q-1}\left|K_{0}^{\prime}\right|^{1 / p^{\prime}}\left|L_{0}^{\prime}\right|^{1 / q^{\prime}} .
$$

Inserting $\left|K_{0}\right|,\left|K_{0}^{\prime}\right|,\left|L_{0}\right|,\left|L_{0}^{\prime}\right| \leq \alpha$ (see (4.15) $)$ into (6.104), we obtain the estimate

$$
\|T\|^{2} \alpha^{1 / p+1 / q} \text {. }
$$

Summary of Case 2 and Case 3. Combining (6.97) with (6.105) yields

$$
\mathbb{E}_{\theta, \varepsilon} Y^{2} \leq\|T\|^{2} \alpha \text {. }
$$

CASE 4, Group (B3) $): K_{0}=K_{1}^{\prime} \neq K_{0}^{\prime}=K_{1}, L_{0}=L_{1} \neq L_{0}^{\prime}=L_{1}^{\prime}(((0)(1))((1)$ (0)) (NIL NIL)) - LEFT VARIANT. In this case, we have to estimate

$$
\sum_{K_{0}, L_{0}^{\prime}, K_{0}^{\prime}, L_{0}}\left\langle T h_{K_{0} \times L_{0}}, h_{K_{0}^{\prime} \times L_{0}^{\prime}}\right\rangle\left\langle T h_{K_{0}^{\prime} \times L_{0}}, h_{K_{0} \times L_{0}^{\prime}}\right\rangle .
$$

We put $a_{K_{0}, K_{0}^{\prime}, L_{0}, L_{0}^{\prime}}=\left\langle T h_{K_{0} \times L_{0}}, h_{K_{0}^{\prime} \times L_{0}^{\prime}}\right\rangle$ and note the estimate

$$
\left|a_{K_{0}, K_{0}^{\prime}, L_{0}, L_{0}^{\prime}}\right| \leq\|T\|\left|K_{0}\right|^{1 / p}\left|L_{0}\right|^{1 / q}\left|K_{0}^{\prime}\right|^{1 / p^{\prime}}\left|L_{0}^{\prime}\right|^{1 / q^{\prime}} .
$$

Now, we write (6.107) as follows:

$$
\sum_{K_{0}, L_{0}^{\prime}}\left\langle T \sum_{K_{0}^{\prime}, L_{0}} a_{K_{0}, K_{0}^{\prime}, L_{0}, L_{0}^{\prime}} h_{K_{0}^{\prime} \times L_{0}}, h_{K_{0} \times L_{0}^{\prime}}\right\rangle .
$$

By duality, we obtain the subsequent upper estimate for (6.109):

$$
\sum_{K_{0}, L_{0}^{\prime}}\left\|T \sum_{K_{0}^{\prime}, L_{0}} a_{K_{0}, K_{0}^{\prime}, L_{0}, L_{0}^{\prime}} h_{K_{0}^{\prime} \times L_{0}}\right\|_{H^{p}\left(H^{q}\right)}\left\|h_{K_{0} \times L_{0}^{\prime}}\right\|_{\left(H^{p}\left(H^{q}\right)\right)^{*}} .
$$


Estimate (6.108) and the disjointness of the dyadic intervals (see (J1)) yield

$$
\sum_{K_{0}, L_{0}^{\prime}}\|T\|\left\|\sum_{K_{0}^{\prime}, L_{0}} \max _{K_{0}^{\prime}, L_{0}}\left(\|T\|\left|K_{0}\right|^{1 / p}\left|L_{0}\right|^{1 / q}\left|K_{0}^{\prime}\right|^{1 / p^{\prime}}\left|L_{0}^{\prime}\right|^{1 / q^{\prime}}\right) h_{K_{0}^{\prime} \times L_{0}}\right\|\left\|_{H^{p}\left(H^{q}\right)}\right\| h_{K_{0} \times L_{0}^{\prime}} \|_{\left(H^{p}\left(H^{q}\right)\right)^{*}} .
$$

Consequently, we obtain

$$
\sum_{K_{0}, L_{0}^{\prime}}\|T\| \max _{K_{0}^{\prime}, L_{0}}\left(\|T\|\left|K_{0}\right|^{1 / p}\left|L_{0}\right|^{1 / q}\left|K_{0}^{\prime}\right|^{1 / p^{\prime}}\left|L_{0}^{\prime}\right|^{1 / q^{\prime}}\right)\left\|\sum_{K_{0}^{\prime}, L_{0}} h_{K_{0}^{\prime} \times L_{0}}\right\|\left\|_{H^{p}\left(H^{q}\right)}\right\| h_{K_{0} \times L_{0}^{\prime}} \|_{\left(H^{p}\left(H^{q}\right)\right)^{*}} .
$$

Thus, 6.112 is bounded from above by

$$
\|T\|^{2} \max _{K_{0}^{\prime}, L_{0}}\left|L_{0}\right|^{1 / q}\left|K_{0}^{\prime}\right|^{1 / p^{\prime}} \sum_{K_{0}, L_{0}^{\prime}}\left|K_{0}\right|\left|L_{0}^{\prime}\right|^{2 / q^{\prime}}
$$

Using Hölder's inequality yields

$$
\|T\|^{2} \max _{K_{0}^{\prime}, L_{0}, L_{0}^{\prime}}\left|L_{0}\right|^{1 / q}\left|K_{0}^{\prime}\right|^{1 / p^{\prime}}\left|L_{0}^{\prime}\right|^{2 / q^{\prime}-1} .
$$

Inserting $\left|K_{0}^{\prime}\right|,\left|L_{0}\right|,\left|L_{0}^{\prime}\right| \leq \alpha$ (see (4.15)) into (6.113), we obtain the estimate

$$
\|T\|^{2} \alpha^{1 / p^{\prime}+1 / q^{\prime}}
$$

CAse 5, Group (B3) $: K_{0}=K_{1}^{\prime} \neq K_{0}^{\prime}=K_{1}, L_{0}=L_{1} \neq L_{0}^{\prime}=L_{1}^{\prime}(((1)(0))$ (NIL NIL) $((0)(1)))$ - RIGHT VARIANT. In this case, we have to estimate

$$
\sum_{K_{0}^{\prime}, L_{0}, K_{0}, L_{0}^{\prime}}\left\langle T h_{K_{0} \times L_{0}}, h_{K_{0}^{\prime} \times L_{0}^{\prime}}\right\rangle\left\langle T h_{K_{0}^{\prime} \times L_{0}}, h_{K_{0} \times L_{0}^{\prime}}\right\rangle .
$$

We put $a_{K_{0}, K_{0}^{\prime}, L_{0}, L_{0}^{\prime}}=\left\langle T h_{K_{0} \times L_{0}}, h_{K_{0}^{\prime} \times L_{0}^{\prime}}\right\rangle$ and note the estimate

$$
\left|a_{K_{0}, K_{0}^{\prime}, L_{0}, L_{0}^{\prime}}\right| \leq\|T\|\left|K_{0}\right|^{1 / p}\left|L_{0}\right|^{1 / q}\left|K_{0}^{\prime}\right|^{1 / p^{\prime}}\left|L_{0}^{\prime}\right|^{1 / q^{\prime}} .
$$

Now, we write (6.115) as follows:

$$
\sum_{K_{0}^{\prime}, L_{0}}\left\langle T h_{K_{0}^{\prime} \times L_{0}}, \sum_{K_{0}, L_{0}^{\prime}} a_{K_{0}, K_{0}^{\prime}, L_{0}, L_{0}^{\prime}} h_{K_{0} \times L_{0}^{\prime}}\right\rangle .
$$

By duality, we obtain the subsequent upper estimate for (6.117):

$$
\sum_{K_{0}^{\prime}, L_{0}}\left\|T h_{K_{0}^{\prime} \times L_{0}}\right\|_{H^{p}\left(H^{q}\right)}\left\|\sum_{K_{0}, L_{0}^{\prime}} a_{K_{0}, K_{0}^{\prime}, L_{0}, L_{0}^{\prime}} h_{K_{0} \times L_{0}^{\prime}}\right\|_{\left(H^{p}\left(H^{q}\right)\right)^{*}} .
$$

Estimate (6.116) and the disjointness of the dyadic intervals (see (J1)) yield

$$
\sum_{K_{0}^{\prime}, L_{0}}\|T\|\left\|h_{K_{0}^{\prime} \times L_{0}}\right\|_{H^{p}\left(H^{q}\right)}\left\|\sum_{K_{0}, L_{0}^{\prime}} \max _{K_{0}, L_{0}^{\prime}}\left(\|T\|\left|K_{0}\right|^{1 / p}\left|L_{0}\right|^{1 / q}\left|K_{0}^{\prime}\right|^{1 / p^{\prime}}\left|L_{0}^{\prime}\right|^{1 / q^{\prime}}\right) h_{K_{0} \times L_{0}^{\prime}}\right\|_{\left(H^{p}\left(H^{q}\right)\right)^{*}} .
$$

Consequently, we obtain

$$
\sum_{K_{0}^{\prime}, L_{0}}\|T\|\left\|h_{K_{0}^{\prime} \times L_{0}}\right\|_{H^{p}\left(H^{q}\right)} \max _{K_{0}, L_{0}^{\prime}}\left(\|T\|\left|K_{0}\right|^{1 / p}\left|L_{0}\right|^{1 / q}\left|K_{0}^{\prime}\right|^{1 / p^{\prime}}\left|L_{0}^{\prime}\right|^{1 / q^{\prime}}\right)\left\|\sum_{K_{0}, L_{0}^{\prime}} h_{K_{0} \times L_{0}^{\prime}}\right\|_{\left(H^{p}\left(H^{q}\right)\right)^{*}} .
$$

Thus, 6.120 is bounded from above by

$$
\|T\|^{2} \max _{K_{0}, L_{0}^{\prime}}\left|K_{0}\right|^{1 / p}\left|L_{0}^{\prime}\right|^{1 / q^{\prime}} \sum_{K_{0}^{\prime}, L_{0}}\left|L_{0}\right|^{2 / q}\left|K_{0}^{\prime}\right| .
$$


DIMENSION DEPENDENCE OF FACTORIZATION PROBLEMS: BI-PARAMETER HARDY SPACES

Using Hölder's inequality yields

$$
\|T\|^{2} \max _{K_{0}, L_{0}, L_{0}^{\prime}}\left|K_{0}\right|^{1 / p}\left|L_{0}^{\prime}\right|^{1 / q^{\prime}}\left|L_{0}\right|^{2 / q-1} .
$$

Inserting $\left|K_{0}\right|,\left|L_{0}\right|,\left|L_{0}^{\prime}\right| \leq \alpha$ (see (4.15)) into (6.121), we obtain the estimate

$$
\|T\|^{2} \alpha^{1 / p+1 / q} \text {. }
$$

Summary of Case 4 and Case 5. Combining (6.114) with (6.122) yields

$$
\mathbb{E}_{\theta, \varepsilon} Y^{2} \leq\|T\|^{2} \alpha
$$

CAse 6, Group (B4) : $K_{0}=K_{0}^{\prime} \neq K_{1}=K_{1}^{\prime}, L_{0}=L_{1} \neq L_{0}^{\prime}=L_{1}^{\prime}(((2)(1))((0)$ (0)) (NIL NIL)) - LEFT VARIANT. In this case, we have to estimate

$$
\sum_{K_{1}, L_{0}^{\prime}, K_{0}, L_{0}}\left\langle T h_{K_{0} \times L_{0}}, h_{K_{0} \times L_{0}^{\prime}}\right\rangle\left\langle T h_{K_{1} \times L_{0}}, h_{K_{1} \times L_{0}^{\prime}}\right\rangle .
$$

We put $a_{K_{0}, L_{0}, L_{0}^{\prime}}=\left\langle T h_{K_{0} \times L_{0}}, h_{K_{0} \times L_{0}^{\prime}}\right\rangle$ and note the estimate

$$
\left|a_{K_{0}, L_{0}, L_{0}^{\prime}}\right| \leq\|T\|\left|K_{0}\right|^{1 / p}\left|L_{0}\right|^{1 / q}\left|K_{0}\right|^{1 / p^{\prime}}\left|L_{0}^{\prime}\right|^{1 / q^{\prime}} .
$$

Now, we write (6.124) as follows:

$$
\sum_{K_{1}, L_{0}^{\prime}}\left\langle T \sum_{K_{0}, L_{0}} a_{K_{0}, L_{0}, L_{0}^{\prime}} h_{K_{1} \times L_{0}}, h_{K_{1} \times L_{0}^{\prime}}\right\rangle .
$$

By duality, we obtain the subsequent upper estimate for (6.126):

$$
\sum_{K_{1}, L_{0}^{\prime}}\left\|T \sum_{K_{0}, L_{0}} a_{K_{0}, L_{0}, L_{0}^{\prime}} h_{K_{1} \times L_{0}}\right\|_{H^{p}\left(H^{q}\right)}\left\|h_{K_{1} \times L_{0}^{\prime}}\right\|_{\left(H^{p}\left(H^{q}\right)\right)^{*}} .
$$

Estimate (6.125) and the disjointness of the dyadic intervals (see (J10) yield

$$
\sum_{K_{1}, L_{0}^{\prime}}\|T\|\left\|\sum_{K_{0}, L_{0}} \max _{L_{0}}\left(\|T\|\left|K_{0}\right|^{1 / p}\left|L_{0}\right|^{1 / q}\left|K_{0}\right|^{1 / p^{\prime}}\left|L_{0}^{\prime}\right|^{1 / q^{\prime}}\right) h_{K_{1} \times L_{0}}\right\|_{H^{p}\left(H^{q}\right)}\left\|h_{K_{1} \times L_{0}^{\prime}}\right\|_{\left(H^{p}\left(H^{q}\right)\right)^{*}} .
$$

Consequently, we obtain

$$
\sum_{K_{1}, L_{0}^{\prime}}\|T\| \sum_{K_{0}} \max _{L_{0}}\left(\|T\|\left|K_{0}\right|^{1 / p}\left|L_{0}\right|^{1 / q}\left|K_{0}\right|^{1 / p^{\prime}}\left|L_{0}^{\prime}\right|^{1 / q^{\prime}}\right)\left\|\sum_{L_{0}} h_{K_{1} \times L_{0}}\right\|_{H^{p}\left(H^{q}\right)}\left\|h_{K_{1} \times L_{0}^{\prime}}\right\|_{\left(H^{p}\left(H^{q}\right)\right)^{*}} .
$$

Thus, (6.129) is bounded from above by

$$
\|T\|^{2} \max _{L_{0}}\left|L_{0}\right|^{1 / q} \sum_{K_{1}, L_{0}^{\prime}} \sum_{K_{0}}\left|K_{0}\right|\left|L_{0}^{\prime}\right|^{2 / q^{\prime}}\left|K_{1}\right| .
$$

Using Hölder's inequality yields

$$
\|T\|^{2} \max _{L_{0}, L_{0}^{\prime}}\left|L_{0}\right|^{1 / q}\left|L_{0}^{\prime}\right|^{2 / q^{\prime}-1}
$$

Inserting $\left|L_{0}\right|,\left|L_{0}^{\prime}\right| \leq \alpha$ (see (4.15) ) into (6.130), we obtain the estimate

$$
\|T\|^{2} \alpha^{1 / q^{\prime}} \text {. }
$$

Case 7, Group (B4) $: K_{0}=K_{0}^{\prime} \neq K_{1}=K_{1}^{\prime}, L_{0}=L_{1} \neq L_{0}^{\prime}=L_{1}^{\prime}(((2)(0))(\mathrm{NIL}$ NIL) $((0)(1)))$ - RIGHT VARIANT. In this case, we have to estimate

$$
\sum_{K_{1}, L_{0}, K_{0}, L_{0}^{\prime}}\left\langle T h_{K_{0} \times L_{0}}, h_{K_{0} \times L_{0}^{\prime}}\right\rangle\left\langle T h_{K_{1} \times L_{0}}, h_{K_{1} \times L_{0}^{\prime}}\right\rangle .
$$


We put $a_{K_{0}, L_{0}, L_{0}^{\prime}}=\left\langle T h_{K_{0} \times L_{0}}, h_{K_{0} \times L_{0}^{\prime}}\right\rangle$ and note the estimate

$$
\left|a_{K_{0}, L_{0}, L_{0}^{\prime}}\right| \leq\|T\|\left|K_{0}\right|^{1 / p}\left|L_{0}\right|^{1 / q}\left|K_{0}\right|^{1 / p^{\prime}}\left|L_{0}^{\prime}\right|^{1 / q^{\prime}} .
$$

Now, we write (6.132) as follows:

$$
\sum_{K_{1}, L_{0}}\left\langle T h_{K_{1} \times L_{0}}, \sum_{K_{0}, L_{0}^{\prime}} a_{K_{0}, L_{0}, L_{0}^{\prime}} h_{K_{1} \times L_{0}^{\prime}}\right\rangle .
$$

By duality, we obtain the subsequent upper estimate for (6.134):

$$
\sum_{K_{1}, L_{0}}\left\|T h_{K_{1} \times L_{0}}\right\|_{H^{p}\left(H^{q}\right)}\left\|\sum_{K_{0}, L_{0}^{\prime}} a_{K_{0}, L_{0}, L_{0}^{\prime}} h_{K_{1} \times L_{0}^{\prime}}\right\|_{\left(H^{p}\left(H^{q}\right)\right)^{*}} .
$$

Estimate (6.133) and the disjointness of the dyadic intervals (see (J1)) yield

$$
\sum_{K_{1}, L_{0}}\|T\|\left\|h_{K_{1} \times L_{0}}\right\|_{H^{p}\left(H^{q}\right)}\left\|\sum_{K_{0}, L_{0}^{\prime}} \max _{L_{0}^{\prime}}\left(\|T\|\left|K_{0}\right|^{1 / p}\left|L_{0}\right|^{1 / q}\left|K_{0}\right|^{1 / p^{\prime}}\left|L_{0}^{\prime}\right|^{1 / q^{\prime}}\right) h_{K_{1} \times L_{0}^{\prime}}\right\|_{\left(H^{p}\left(H^{q}\right)\right)^{*}} .
$$

Consequently, we obtain

$$
\sum_{K_{1}, L_{0}}\|T\|\left\|h_{K_{1} \times L_{0}}\right\|_{H^{p}\left(H^{q}\right)} \sum_{K_{0}} \max _{L_{0}^{\prime}}\left(\|T\|\left|K_{0}\right|^{1 / p}\left|L_{0}\right|^{1 / q}\left|K_{0}\right|^{1 / p^{\prime}}\left|L_{0}^{\prime}\right|^{1 / q^{\prime}}\right)\left\|\sum_{L_{0}^{\prime}} h_{K_{1} \times L_{0}^{\prime}}\right\|_{\left(H^{p}\left(H^{q}\right)\right)^{*}} .
$$

Thus, (6.137) is bounded from above by

$$
\|T\|^{2} \max _{L_{0}^{\prime}}\left|L_{0}^{\prime}\right|^{1 / q^{\prime}} \sum_{K_{1}, L_{0}} \sum_{K_{0}}\left|K_{0} \| K_{1}\right|\left|L_{0}\right|^{2 / q}
$$

Using Hölder's inequality yields

$$
\|T\|^{2} \max _{L_{0}, L_{0}^{\prime}}\left|L_{0}^{\prime}\right|^{1 / q^{\prime}}\left|L_{0}\right|^{2 / q-1} .
$$

Inserting $\left|L_{0}\right|,\left|L_{0}^{\prime}\right| \leq \alpha$ (see (4.15)) into (6.138), we obtain the estimate

$$
\|T\|^{2} \alpha^{1 / q} \text {. }
$$

Summary of Case 6 and Case 7. Combining (6.131) with (6.139) yields

$$
\mathbb{E}_{\theta, \varepsilon} Y^{2} \leq\|T\|^{2} \alpha^{1 / 2} .
$$

Summary for $Y$. Combining (6.89) with (6.106), (6.123) and (6.140) yields

$$
\mathbb{E}_{\theta, \varepsilon} Y^{2} \leq 4\|T\|^{2} \alpha^{1 / 2}
$$

6.4. Estimates for $Z$. In this case, the following variables will always be summed over the following sets:

$$
\begin{aligned}
& \triangleright K_{0}, K_{1}, K_{0}^{\prime}, K_{1}^{\prime} \text { over } X_{I}, \\
& \triangleright L_{0}, L_{1}, L_{0}^{\prime}, L_{1}^{\prime} \text { over } y_{J},
\end{aligned}
$$

such that

$$
\left(K_{0} \neq K_{0}^{\prime} \quad \text { or } \quad L_{0} \neq L_{0}^{\prime}\right) \quad \text { and } \quad\left(K_{1} \neq K_{1}^{\prime} \quad \text { or } \quad L_{1} \neq L_{1}^{\prime}\right) .
$$


Proof. Note that by (4.14d) and (4.13), we obtain $Z^{2}(\theta, \varepsilon)$ is given by

$$
\sum_{\substack{K_{0}, K_{1}, K_{0}^{\prime}, K_{1}^{\prime} \\ L_{0}, L_{1}, L_{0}^{\prime}, L_{1}^{\prime}}} \theta_{K_{0}} \theta_{K_{1}} \theta_{K_{0}^{\prime}} \theta_{K_{1}^{\prime}} \varepsilon_{L_{0}} \varepsilon_{L_{1}} \varepsilon_{L_{0}^{\prime}} \varepsilon_{L_{1}^{\prime}}\left\langle T h_{K_{0} \times L_{0}}, h_{K_{0}^{\prime} \times L_{0}^{\prime}}\right\rangle\left\langle T h_{K_{1} \times L_{1}}, h_{K_{1}^{\prime} \times L_{1}^{\prime}}\right\rangle
$$

Hence, in view of (R1)-(R4), we decompose the index set in (6.81) into the following fifteen groups:

(d1) $K_{0}=K_{1}=K_{0}^{\prime}=K_{1}^{\prime}$ and $L_{0}=L_{1} \neq L_{0}^{\prime}=L_{1}^{\prime}$;

(d2) $K_{0}=K_{1}=K_{0}^{\prime}=K_{1}^{\prime}$ and $L_{0}=L_{1}^{\prime} \neq L_{0}^{\prime}=L_{1}$;

(d3) $K_{0}=K_{1}=K_{0}^{\prime}=K_{1}^{\prime}$ and $L_{0}=L_{0}^{\prime} \neq L_{1}=L_{1}^{\prime}$ (excluded by (6.142) );

(e1) $K_{0}=K_{1} \neq K_{0}^{\prime}=K_{1}^{\prime}$ and $L_{0}=L_{1}=L_{0}^{\prime}=L_{1}^{\prime}$;

(e2) $K_{0}=K_{1}^{\prime} \neq K_{0}^{\prime}=K_{1}$ and $L_{0}=L_{1}=L_{0}^{\prime}=L_{1}^{\prime}$;

(e3) $K_{0}=K_{0}^{\prime} \neq K_{1}=K_{1}^{\prime}$ and $L_{0}=L_{1}=L_{0}^{\prime}=L_{1}^{\prime}$ (excluded by (6.142) );

(f1) $K_{0}=K_{1} \neq K_{0}^{\prime}=K_{1}^{\prime}$ and $L_{0}=L_{1} \neq L_{0}^{\prime}=L_{1}^{\prime}$;

(f2) $K_{0}=K_{1} \neq K_{0}^{\prime}=K_{1}^{\prime}$ and $L_{0}=L_{1}^{\prime} \neq L_{0}^{\prime}=L_{1}$;

(f3) $K_{0}=K_{1} \neq K_{0}^{\prime}=K_{1}^{\prime}$ and $L_{0}=L_{0}^{\prime} \neq L_{1}=L_{1}^{\prime}$;

(f4) $K_{0}=K_{1}^{\prime} \neq K_{0}^{\prime}=K_{1}$ and $L_{0}=L_{1} \neq L_{0}^{\prime}=L_{1}^{\prime}$;

(f5) $K_{0}=K_{1}^{\prime} \neq K_{0}^{\prime}=K_{1}$ and $L_{0}=L_{1}^{\prime} \neq L_{0}^{\prime}=L_{1}$;

(f6) $K_{0}=K_{1}^{\prime} \neq K_{0}^{\prime}=K_{1}$ and $L_{0}=L_{0}^{\prime} \neq L_{1}=L_{1}^{\prime}$;

(f7) $K_{0}=K_{0}^{\prime} \neq K_{1}=K_{1}^{\prime}$ and $L_{0}=L_{1} \neq L_{0}^{\prime}=L_{1}^{\prime}$;

(f8) $K_{0}=K_{0}^{\prime} \neq K_{1}=K_{1}^{\prime}$ and $L_{0}=L_{1}^{\prime} \neq L_{0}^{\prime}=L_{1}$;

(f9) $K_{0}=K_{0}^{\prime} \neq K_{1}=K_{1}^{\prime}$ and $L_{0}=L_{0}^{\prime} \neq L_{1}=L_{1}^{\prime}$ (excluded by (6.142) ).

As we indicated above, the cases (d1), (e1) and (f1) are contradicting the constraint (6.142), and are thereby excluded.

CAse 1, Group (D1): $K_{0}=K_{0}^{\prime}=K_{1}=K_{1}^{\prime}, L_{0}=L_{1} \neq L_{0}^{\prime}=L_{1}^{\prime}(((0)(1))$ (NIL (0)) (NIL NIL)) - LEFT VARIANT. In this case, we have to estimate

$$
\sum_{K_{0}, L_{0}^{\prime}, L_{0}}\left\langle T h_{K_{0} \times L_{0}}, h_{K_{0} \times L_{0}^{\prime}}\right\rangle\left\langle T h_{K_{0} \times L_{0}}, h_{K_{0} \times L_{0}^{\prime}}\right\rangle .
$$

We put $a_{K_{0}, L_{0}, L_{0}^{\prime}}=\left\langle T h_{K_{0} \times L_{0}}, h_{K_{0} \times L_{0}^{\prime}}\right\rangle$ and note the estimate

$$
\left|a_{K_{0}, L_{0}, L_{0}^{\prime}}\right| \leq\|T\|\left|K_{0}\right|^{1 / p}\left|L_{0}\right|^{1 / q}\left|K_{0}\right|^{1 / p^{\prime}}\left|L_{0}^{\prime}\right|^{1 / q^{\prime}} .
$$

Now, we write 6.144) as follows:

$$
\sum_{K_{0}, L_{0}^{\prime}}\left\langle T \sum_{L_{0}} a_{K_{0}, L_{0}, L_{0}^{\prime}} h_{K_{0} \times L_{0}}, h_{K_{0} \times L_{0}^{\prime}}\right\rangle .
$$

By duality, we obtain the subsequent upper estimate for 6.146):

$$
\sum_{K_{0}, L_{0}^{\prime}}\left\|T \sum_{L_{0}} a_{K_{0}, L_{0}, L_{0}^{\prime}} h_{K_{0} \times L_{0}}\right\|_{H^{p}\left(H^{q}\right)}\left\|h_{K_{0} \times L_{0}^{\prime}}\right\|_{\left(H^{p}\left(H^{q}\right)\right)^{*}} \cdot
$$

Estimate (6.145) and the disjointness of the dyadic intervals (see (JJ1) yield

$$
\sum_{K_{0}, L_{0}^{\prime}}\|T\|\left\|\sum_{L_{0}} \max _{L_{0}}\left(\|T\|\left|K_{0}\right|^{1 / p}\left|L_{0}\right|^{1 / q}\left|K_{0}\right|^{1 / p^{\prime}}\left|L_{0}^{\prime}\right|^{1 / q^{\prime}}\right) h_{K_{0} \times L_{0}}\right\|_{H^{p}\left(H^{q}\right)}\left\|h_{K_{0} \times L_{0}^{\prime}}\right\|_{\left(H^{p}\left(H^{q}\right)\right)^{*}} .
$$

Consequently, we obtain

$$
\sum_{K_{0}, L_{0}^{\prime}}\|T\| \max _{L_{0}}\left(\|T\|\left|K_{0}\right|^{1 / p}\left|L_{0}\right|^{1 / q}\left|K_{0}\right|^{1 / p^{\prime}}\left|L_{0}^{\prime}\right|^{1 / q^{\prime}}\right)\left\|\sum_{L_{0}} h_{K_{0} \times L_{0}}\right\|_{H^{p}\left(H^{q}\right)}\left\|h_{K_{0} \times L_{0}^{\prime}}\right\|_{\left(H^{p}\left(H^{q}\right)\right)^{*}} .
$$


Thus, (6.149) is bounded from above by

$$
\|T\|^{2} \max _{L_{0}}\left|L_{0}\right|^{1 / q} \sum_{K_{0}, L_{0}^{\prime}}\left|K_{0}\right|^{2}\left|L_{0}^{\prime}\right|^{2 / q^{\prime}} .
$$

Using Hölder's inequality yields

$$
\|T\|^{2} \max _{K_{0}, L_{0}, L_{0}^{\prime}}\left|K_{0}\right|\left|L_{0}\right|^{1 / q}\left|L_{0}^{\prime}\right|^{2 / q^{\prime}-1} .
$$

Inserting $\left|K_{0}\right|,\left|L_{0}\right|,\left|L_{0}^{\prime}\right| \leq \alpha$ (see (4.15D) into (6.150), we obtain the estimate

$$
\|T\|^{2} \alpha^{1+1 / q^{\prime}} \text {. }
$$

CASE 2, GROUP (D2): $K_{0}=K_{0}^{\prime}=K_{1}=K_{1}^{\prime}, L_{0}=L_{1}^{\prime} \neq L_{0}^{\prime}=L_{1}(((0)(0))$ (NIL (1)) (NIL NIL)) - LEFT VARIANT. In this case, we have to estimate

$$
\sum_{K_{0}, L_{0}, L_{0}^{\prime}}\left\langle T h_{K_{0} \times L_{0}}, h_{K_{0} \times L_{0}^{\prime}}\right\rangle\left\langle T h_{K_{0} \times L_{0}^{\prime}}, h_{K_{0} \times L_{0}}\right\rangle .
$$

We put $a_{K_{0}, L_{0}, L_{0}^{\prime}}=\left\langle T h_{K_{0} \times L_{0}}, h_{K_{0} \times L_{0}^{\prime}}\right\rangle$ and note the estimate

$$
\left|a_{K_{0}, L_{0}, L_{0}^{\prime}}\right| \leq\|T\|\left|K_{0}\right|^{1 / p}\left|L_{0}\right|^{1 / q}\left|K_{0}\right|^{1 / p^{\prime}}\left|L_{0}^{\prime}\right|^{1 / q^{\prime}} .
$$

Now, we write (6.152) as follows:

$$
\sum_{K_{0}, L_{0}}\left\langle T \sum_{L_{0}^{\prime}} a_{K_{0}, L_{0}, L_{0}^{\prime}} h_{K_{0} \times L_{0}^{\prime}}, h_{K_{0} \times L_{0}}\right\rangle .
$$

By duality, we obtain the subsequent upper estimate for (6.154):

$$
\sum_{K_{0}, L_{0}}\left\|T \sum_{L_{0}^{\prime}} a_{K_{0}, L_{0}, L_{0}^{\prime}} h_{K_{0} \times L_{0}^{\prime}}\right\|_{H^{p}\left(H^{q}\right)}\left\|h_{K_{0} \times L_{0}}\right\|_{\left(H^{p}\left(H^{q}\right)\right)^{*}} .
$$

Estimate (6.153) and the disjointness of the dyadic intervals (see (JJ1) yield

$$
\sum_{K_{0}, L_{0}}\|T\|\left\|\sum_{L_{0}^{\prime}} \max _{L_{0}^{\prime}}\left(\|T\|\left|K_{0}\right|^{1 / p}\left|L_{0}\right|^{1 / q}\left|K_{0}\right|^{1 / p^{\prime}}\left|L_{0}^{\prime}\right|^{1 / q^{\prime}}\right) h_{K_{0} \times L_{0}^{\prime}}\right\|_{H^{p}\left(H^{q}\right)}\left\|h_{K_{0} \times L_{0}}\right\|_{\left(H^{p}\left(H^{q}\right)\right)^{*}} .
$$

Consequently, we obtain

$$
\sum_{K_{0}, L_{0}}\|T\| \max _{L_{0}^{\prime}}\left(\|T\|\left|K_{0}\right|^{1 / p}\left|L_{0}\right|^{1 / q}\left|K_{0}\right|^{1 / p^{\prime}}\left|L_{0}^{\prime}\right|^{1 / q^{\prime}}\right)\left\|\sum_{L_{0}^{\prime}} h_{K_{0} \times L_{0}^{\prime}}\right\|_{H^{p}\left(H^{q}\right)}\left\|h_{K_{0} \times L_{0}}\right\|_{\left(H^{p}\left(H^{q}\right)\right)^{*}} .
$$

Thus, (6.157) is bounded from above by

$$
\|T\|^{2} \max _{L_{0}^{\prime}}\left|L_{0}^{\prime}\right|^{1 / q^{\prime}} \sum_{K_{0}, L_{0}}\left|K_{0}\right|^{2}\left|L_{0}\right|
$$

Using Hölder's inequality yields

$$
\|T\|^{2} \max _{K_{0}, L_{0}, L_{0}^{\prime}}\left|L_{0}^{\prime}\right|^{1 / q^{\prime}}\left|K_{0}\right| .
$$

Inserting $\left|K_{0}\right|,\left|L_{0}\right|,\left|L_{0}^{\prime}\right| \leq \alpha$ (see (4.15)) into (6.158), we obtain the estimate

$$
\|T\|^{2} \alpha^{1+1 / q^{\prime}} \text {. }
$$


CASE 3, GRoup (E1): $K_{0}=K_{1} \neq K_{0}^{\prime}=K_{1}^{\prime}, L_{0}=L_{0}^{\prime}=L_{1}=L_{1}^{\prime}(((1)(0))((0)$ NIL) (NIL NIL)) - LEFT VARIANT. In this case, we have to estimate

$$
\sum_{K_{0}^{\prime}, L_{0}, K_{0}}\left\langle T h_{K_{0} \times L_{0}}, h_{K_{0}^{\prime} \times L_{0}}\right\rangle\left\langle T h_{K_{0} \times L_{0}}, h_{K_{0}^{\prime} \times L_{0}}\right\rangle .
$$

We put $a_{K_{0}, K_{0}^{\prime}, L_{0}}=\left\langle T h_{K_{0} \times L_{0}}, h_{K_{0}^{\prime} \times L_{0}}\right\rangle$ and note the estimate

$$
\left|a_{K_{0}, K_{0}^{\prime}, L_{0}}\right| \leq\|T\|\left|K_{0}\right|^{1 / p}\left|L_{0}\right|^{1 / q}\left|K_{0}^{\prime}\right|^{1 / p^{\prime}}\left|L_{0}\right|^{1 / q^{\prime}} .
$$

Now, we write (6.160) as follows:

$$
\sum_{K_{0}^{\prime}, L_{0}}\left\langle T \sum_{K_{0}} a_{K_{0}, K_{0}^{\prime}, L_{0}} h_{K_{0} \times L_{0}}, h_{K_{0}^{\prime} \times L_{0}}\right\rangle .
$$

By duality, we obtain the subsequent upper estimate for (6.162):

$$
\sum_{K_{0}^{\prime}, L_{0}}\left\|T \sum_{K_{0}} a_{K_{0}, K_{0}^{\prime}, L_{0}} h_{K_{0} \times L_{0}}\right\|_{H^{p}\left(H^{q}\right)}\left\|h_{K_{0}^{\prime} \times L_{0}}\right\|_{\left(H^{p}\left(H^{q}\right)\right)^{*}} .
$$

Estimate (6.161) and the disjointness of the dyadic intervals (see (J1)) yield

$$
\sum_{K_{0}^{\prime}, L_{0}}\|T\|\left\|\sum_{K_{0}} \max _{K_{0}}\left(\|T\|\left|K_{0}\right|^{1 / p}\left|L_{0}\right|^{1 / q}\left|K_{0}^{\prime}\right|^{1 / p^{\prime}}\left|L_{0}\right|^{1 / q^{\prime}}\right) h_{K_{0} \times L_{0}}\right\|_{H^{p}\left(H^{q}\right)}\left\|h_{K_{0}^{\prime} \times L_{0}}\right\|_{\left(H^{p}\left(H^{q}\right)\right)^{*}} .
$$

Consequently, we obtain

$$
\sum_{K_{0}^{\prime}, L_{0}}\|T\| \max _{K_{0}}\left(\|T\|\left|K_{0}\right|^{1 / p}\left|L_{0}\right|^{1 / q}\left|K_{0}^{\prime}\right|^{1 / p^{\prime}}\left|L_{0}\right|^{1 / q^{\prime}}\right)\left\|\sum_{K_{0}} h_{K_{0} \times L_{0}}\right\|_{H^{p}\left(H^{q}\right)}\left\|h_{K_{0}^{\prime} \times L_{0}}\right\|_{\left(H^{p}\left(H^{q}\right)\right)^{*}} .
$$

Thus, 6.165) is bounded from above by

$$
\|T\|^{2} \max _{K_{0}}\left|K_{0}\right|^{1 / p} \sum_{K_{0}^{\prime}, L_{0}}\left|L_{0}\right|^{2}\left|K_{0}^{\prime}\right|^{2 / p^{\prime}}
$$

Using Hölder's inequality yields

$$
\|T\|^{2} \max _{K_{0}, K_{0}^{\prime}, L_{0}}\left|K_{0}\right|^{1 / p}\left|L_{0}\right|\left|K_{0}^{\prime}\right|^{2 / p^{\prime}-1} .
$$

Inserting $\left|K_{0}\right|,\left|K_{0}^{\prime}\right|,\left|L_{0}\right| \leq \alpha$ (see (4.15)) into (6.166), we obtain the estimate

$$
\|T\|^{2} \alpha^{1+1 / p^{\prime}} \text {. }
$$

CASE 4, Group (E2): $K_{0}=K_{1}^{\prime} \neq K_{0}^{\prime}=K_{1}, L_{0}=L_{0}^{\prime}=L_{1}=L_{1}^{\prime}(((0)(0))((1)$ NIL) (NIL NIL)) - LEFT VARIANT. In this case, we have to estimate

$$
\sum_{K_{0}, L_{0}, K_{0}^{\prime}}\left\langle T h_{K_{0} \times L_{0}}, h_{K_{0}^{\prime} \times L_{0}}\right\rangle\left\langle T h_{K_{0}^{\prime} \times L_{0}}, h_{K_{0} \times L_{0}}\right\rangle .
$$

We put $a_{K_{0}, K_{0}^{\prime}, L_{0}}=\left\langle T h_{K_{0} \times L_{0}}, h_{K_{0}^{\prime} \times L_{0}}\right\rangle$ and note the estimate

$$
\left|a_{K_{0}, K_{0}^{\prime}, L_{0}}\right| \leq \| T||\left|K_{0}\right|^{1 / p}\left|L_{0}\right|^{1 / q}\left|K_{0}^{\prime}\right|^{1 / p^{\prime}}\left|L_{0}\right|^{1 / q^{\prime}} .
$$

Now, we write (6.168) as follows:

$$
\sum_{K_{0}, L_{0}}\left\langle T \sum_{K_{0}^{\prime}} a_{K_{0}, K_{0}^{\prime}, L_{0}} h_{K_{0}^{\prime} \times L_{0}}, h_{K_{0} \times L_{0}}\right\rangle .
$$


By duality, we obtain the subsequent upper estimate for (6.170):

$$
\sum_{K_{0}, L_{0}}\left\|T \sum_{K_{0}^{\prime}} a_{K_{0}, K_{0}^{\prime}, L_{0}} h_{K_{0}^{\prime} \times L_{0}}\right\|_{H^{p}\left(H^{q}\right)}\left\|h_{K_{0} \times L_{0}}\right\|_{\left(H^{p}\left(H^{q}\right)\right)^{*}} .
$$

Estimate (6.169) and the disjointness of the dyadic intervals (see (JJ1)) yield

$$
\sum_{K_{0}, L_{0}}\|T\|\left\|\sum_{K_{0}^{\prime}} \max _{K_{0}^{\prime}}\left(\|T\|\left|K_{0}\right|^{1 / p}\left|L_{0}\right|^{1 / q}\left|K_{0}^{\prime}\right|^{1 / p^{\prime}}\left|L_{0}\right|^{1 / q^{\prime}}\right) h_{K_{0}^{\prime} \times L_{0}}\right\|_{H^{p}\left(H^{q}\right)}\left\|h_{K_{0} \times L_{0}}\right\|_{\left(H^{p}\left(H^{q}\right)\right)^{*}} .
$$

Consequently, we obtain

$$
\sum_{K_{0}, L_{0}}\|T\| \max _{K_{0}^{\prime}}\left(\|T\|\left|K_{0}\right|^{1 / p}\left|L_{0}\right|^{1 / q}\left|K_{0}^{\prime}\right|^{1 / p^{\prime}}\left|L_{0}\right|^{1 / q^{\prime}}\right)\left\|\sum_{K_{0}^{\prime}} h_{K_{0}^{\prime} \times L_{0}}\right\|_{H^{p}\left(H^{q}\right)}\left\|h_{K_{0} \times L_{0}}\right\|_{\left(H^{p}\left(H^{q}\right)\right)^{*}} .
$$

Thus, 6.173) is bounded from above by

$$
\|T\|^{2} \max _{K_{0}^{\prime}}\left|K_{0}^{\prime}\right|^{1 / p^{\prime}} \sum_{K_{0}, L_{0}}\left|K_{0}\right|\left|L_{0}\right|^{2}
$$

Using Hölder's inequality yields

$$
\|T\|^{2} \max _{K_{0}^{\prime}, L_{0}}\left|K_{0}^{\prime}\right|^{1 / p^{\prime}}\left|L_{0}\right|
$$

Inserting $\left|K_{0}^{\prime}\right|,\left|L_{0}^{\prime}\right| \leq \alpha$ (see (4.15) ) into (6.174), we obtain the estimate

$$
\|T\|^{2} \alpha^{1+1 / p^{\prime}} .
$$

CASE 5, GROUP (F1) : $K_{0}=K_{1} \neq K_{0}^{\prime}=K_{1}^{\prime}, L_{0}=L_{1} \neq L_{0}^{\prime}=L_{1}^{\prime}(((1)(1))((0)$ (0)) (NIL NIL)) - LEFT VARIANT. In this case, we have to estimate

$$
\sum_{K_{0}^{\prime}, L_{0}^{\prime}, K_{0}, L_{0}}\left\langle T h_{K_{0} \times L_{0}}, h_{K_{0}^{\prime} \times L_{0}^{\prime}}\right\rangle\left\langle T h_{K_{0} \times L_{0}}, h_{K_{0}^{\prime} \times L_{0}^{\prime}}\right\rangle .
$$

We put $a_{K_{0}, K_{0}^{\prime}, L_{0}, L_{0}^{\prime}}=\left\langle T h_{K_{0} \times L_{0}}, h_{K_{0}^{\prime} \times L_{0}^{\prime}}\right\rangle$ and note the estimate

$$
\left|a_{K_{0}, K_{0}^{\prime}, L_{0}, L_{0}^{\prime}}\right| \leq\|T\|\left|K_{0}\right|^{1 / p}\left|L_{0}\right|^{1 / q}\left|K_{0}^{\prime}\right|^{1 / p^{\prime}}\left|L_{0}^{\prime}\right|^{1 / q^{\prime}} .
$$

Now, we write (6.176) as follows:

$$
\sum_{K_{0}^{\prime}, L_{0}^{\prime}}\left\langle T \sum_{K_{0}, L_{0}} a_{K_{0}, K_{0}^{\prime}, L_{0}, L_{0}^{\prime}} h_{K_{0} \times L_{0}}, h_{K_{0}^{\prime} \times L_{0}^{\prime}}\right\rangle .
$$

By duality, we obtain the subsequent upper estimate for (6.178):

$$
\sum_{K_{0}^{\prime}, L_{0}^{\prime}}\left\|T \sum_{K_{0}, L_{0}} a_{K_{0}, K_{0}^{\prime}, L_{0}, L_{0}^{\prime}} h_{K_{0} \times L_{0}}\right\|_{H^{p}\left(H^{q}\right)}\left\|h_{K_{0}^{\prime} \times L_{0}^{\prime}}\right\|_{\left(H^{p}\left(H^{q}\right)\right)^{*}} \cdot
$$

Estimate (6.177) and the disjointness of the dyadic intervals (see (JJ1)) yield

$$
\sum_{K_{0}^{\prime}, L_{0}^{\prime}}\|T\|\left\|\sum_{K_{0}, L_{0}} \max _{K_{0}, L_{0}}\left(\|T\|\left|K_{0}\right|^{1 / p}\left|L_{0}\right|^{1 / q}\left|K_{0}^{\prime}\right|^{1 / p^{\prime}}\left|L_{0}^{\prime}\right|^{1 / q^{\prime}}\right) h_{K_{0} \times L_{0}}\right\|_{H^{p}\left(H^{q}\right)}\left\|h_{K_{0}^{\prime} \times L_{0}^{\prime}}\right\|_{\left(H^{p}\left(H^{q}\right)\right)^{*}} .
$$


DIMENSION DEPENDENCE OF FACTORIZATION PROBLEMS: BI-PARAMETER HARDY SPACEM

Consequently, we obtain

$\sum_{K_{0}^{\prime}, L_{0}^{\prime}}\|T\| \max _{K_{0}, L_{0}}\left(\|T\|\left|K_{0}\right|^{1 / p}\left|L_{0}\right|^{1 / q}\left|K_{0}^{\prime}\right|^{1 / p^{\prime}}\left|L_{0}^{\prime}\right|^{1 / q^{\prime}}\right)\left\|\sum_{K_{0}, L_{0}} h_{K_{0} \times L_{0}}\right\|\left\|_{H^{p}\left(H^{q}\right)}\right\| h_{K_{0}^{\prime} \times L_{0}^{\prime}} \|_{\left(H^{p}\left(H^{q}\right)\right)^{*}}$.

Thus, (6.181) is bounded from above by

$$
\|T\|^{2} \max _{K_{0}, L_{0}}\left|K_{0}\right|^{1 / p}\left|L_{0}\right|^{1 / q} \sum_{K_{0}^{\prime}, L_{0}^{\prime}}\left|K_{0}^{\prime}\right|^{2 / p^{\prime}}\left|L_{0}^{\prime}\right|^{2 / q^{\prime}} .
$$

Using Hölder's inequality yields

$$
\|T\|^{2} \max _{K_{0}, L_{0}, K_{0}^{\prime}, L_{0}^{\prime}}\left|K_{0}\right|^{1 / p}\left|L_{0}\right|^{1 / q}\left|K_{0}^{\prime}\right|^{2 / p^{\prime}-1}\left|L_{0}^{\prime}\right|^{2 / q^{\prime}-1} .
$$

Inserting $\left|K_{0}\right|,\left|K_{0}^{\prime}\right|,\left|L_{0}\right|,\left|L_{0}^{\prime}\right| \leq \alpha$ (see (4.15) $)$ into (6.182), we obtain the estimate

$$
\|T\|^{2} \alpha^{1 / p^{\prime}+1 / q^{\prime}}
$$

Case 6, Group (F1) : $K_{0}=K_{1} \neq K_{0}^{\prime}=K_{1}^{\prime}, L_{0}=L_{1} \neq L_{0}^{\prime}=L_{1}^{\prime}(((0)(0))$ (NIL NIL) $((1)(1)))$ - RIGHT VARIANT. In this case, we have to estimate

$$
\sum_{K_{0}, L_{0}, K_{0}^{\prime}, L_{0}^{\prime}}\left\langle T h_{K_{0} \times L_{0}}, h_{K_{0}^{\prime} \times L_{0}^{\prime}}\right\rangle\left\langle T h_{K_{0} \times L_{0}}, h_{K_{0}^{\prime} \times L_{0}^{\prime}}\right\rangle .
$$

We put $a_{K_{0}, K_{0}^{\prime}, L_{0}, L_{0}^{\prime}}=\left\langle T h_{K_{0} \times L_{0}}, h_{K_{0}^{\prime} \times L_{0}^{\prime}}\right\rangle$ and note the estimate

$$
\left|a_{K_{0}, K_{0}^{\prime}, L_{0}, L_{0}^{\prime}}\right| \leq\|T\|\left|K_{0}\right|^{1 / p}\left|L_{0}\right|^{1 / q}\left|K_{0}^{\prime}\right|^{1 / p^{\prime}}\left|L_{0}^{\prime}\right|^{1 / q^{\prime}} .
$$

Now, we write (6.184) as follows:

$$
\sum_{K_{0}, L_{0}}\left\langle T h_{K_{0} \times L_{0}}, \sum_{K_{0}^{\prime}, L_{0}^{\prime}} a_{K_{0}, K_{0}^{\prime}, L_{0}, L_{0}^{\prime}} h_{K_{0}^{\prime} \times L_{0}^{\prime}}\right\rangle .
$$

By duality, we obtain the subsequent upper estimate for (6.186):

$$
\sum_{K_{0}, L_{0}}\left\|T h_{K_{0} \times L_{0}}\right\|_{H^{p}\left(H^{q}\right)}\left\|\sum_{K_{0}^{\prime}, L_{0}^{\prime}} a_{K_{0}, K_{0}^{\prime}, L_{0}, L_{0}^{\prime}} h_{K_{0}^{\prime} \times L_{0}^{\prime}}\right\|_{\left(H^{p}\left(H^{q}\right)\right)^{*}} .
$$

Estimate (6.185) and the disjointness of the dyadic intervals (see (JID) yield

$$
\sum_{K_{0}, L_{0}}\|T\|\left\|h_{K_{0} \times L_{0}}\right\|_{H^{p}\left(H^{q}\right)}\left\|\sum_{K_{0}^{\prime}, L_{0}^{\prime}} \max _{K_{0}^{\prime}, L_{0}^{\prime}}\left(\|T\|\left|K_{0}\right|^{1 / p}\left|L_{0}\right|^{1 / q}\left|K_{0}^{\prime}\right|^{1 / p^{\prime}}\left|L_{0}^{\prime}\right|^{1 / q^{\prime}}\right) h_{K_{0}^{\prime} \times L_{0}^{\prime}}\right\|_{\left(H^{p}\left(H^{q}\right)\right)^{*}} .
$$

Consequently, we obtain

$$
\sum_{K_{0}, L_{0}}\|T\|\left\|h_{K_{0} \times L_{0}}\right\|_{H^{p}\left(H^{q}\right)} \max _{K_{0}^{\prime}, L_{0}^{\prime}}\left(\|T\|\left|K_{0}\right|^{1 / p}\left|L_{0}\right|^{1 / q}\left|K_{0}^{\prime}\right|^{1 / p^{\prime}}\left|L_{0}^{\prime}\right|^{1 / q^{\prime}}\right)\left\|\sum_{K_{0}^{\prime}, L_{0}^{\prime}} h_{K_{0}^{\prime} \times L_{0}^{\prime}}\right\|_{\left(H^{p}\left(H^{q}\right)\right)^{*}} .
$$

Thus, 6.189) is bounded from above by

$$
\|T\|^{2} \max _{K_{0}^{\prime}, L_{0}^{\prime}}\left|K_{0}^{\prime}\right|^{1 / p^{\prime}}\left|L_{0}^{\prime}\right|^{1 / q^{\prime}} \sum_{K_{0}, L_{0}}\left|K_{0}\right|^{2 / p}\left|L_{0}\right|^{2 / q} .
$$

Using Hölder's inequality yields

$$
\|T\|^{2} \max _{K_{0}, L_{0}, K_{0}^{\prime}, L_{0}^{\prime}}\left|K_{0}^{\prime}\right|^{1 / p^{\prime}}\left|L_{0}^{\prime}\right|^{1 / q^{\prime}}\left|K_{0}\right|^{2 / p-1}\left|L_{0}\right|^{2 / q-1} .
$$


Inserting $\left|K_{0}\right|,\left|K_{0}^{\prime}\right|,\left|L_{0}\right|,\left|L_{0}^{\prime}\right| \leq \alpha$ (see (4.15) ) into (6.190), we obtain the estimate

$$
\|T\|^{2} \alpha^{1 / p+1 / q} \text {. }
$$

Summary of Case 5 and Case 6. Combining (6.183) with 6.191) yields

$$
\mathbb{E}_{\theta, \varepsilon} Z^{2} \leq\|T\|^{2} \alpha \text {. }
$$

Case 7, Group (F2) $: K_{0}=K_{1} \neq K_{0}^{\prime}=K_{1}^{\prime}, L_{0}=L_{1}^{\prime} \neq L_{0}^{\prime}=L_{1}(((1)(0))((0)$ (1)) (NIL NIL)) - LEFT VARIANT. In this case, we have to estimate

$$
\sum_{K_{0}^{\prime}, L_{0}, K_{0}, L_{0}^{\prime}}\left\langle T h_{K_{0} \times L_{0}}, h_{K_{0}^{\prime} \times L_{0}^{\prime}}\right\rangle\left\langle T h_{K_{0} \times L_{0}^{\prime}}, h_{K_{0}^{\prime} \times L_{0}}\right\rangle .
$$

We put $a_{K_{0}, K_{0}^{\prime}, L_{0}, L_{0}^{\prime}}=\left\langle T h_{K_{0} \times L_{0}}, h_{K_{0}^{\prime} \times L_{0}^{\prime}}\right\rangle$ and note the estimate

$$
\left|a_{K_{0}, K_{0}^{\prime}, L_{0}, L_{0}^{\prime}}\right| \leq\|T\|\left|K_{0}\right|^{1 / p}\left|L_{0}\right|^{1 / q}\left|K_{0}^{\prime}\right|^{1 / p^{\prime}}\left|L_{0}^{\prime}\right|^{1 / q^{\prime}} .
$$

Now, we write (6.193) as follows:

$$
\sum_{K_{0}^{\prime}, L_{0}}\left\langle T \sum_{K_{0}, L_{0}^{\prime}} a_{K_{0}, K_{0}^{\prime}, L_{0}, L_{0}^{\prime}} h_{K_{0} \times L_{0}^{\prime}}, h_{K_{0}^{\prime} \times L_{0}}\right\rangle .
$$

By duality, we obtain the subsequent upper estimate for (6.195):

$$
\sum_{K_{0}^{\prime}, L_{0}}\left\|T \sum_{K_{0}, L_{0}^{\prime}} a_{K_{0}, K_{0}^{\prime}, L_{0}, L_{0}^{\prime}} h_{K_{0} \times L_{0}^{\prime}}\right\|_{H^{p}\left(H^{q}\right)}\left\|h_{K_{0}^{\prime} \times L_{0}}\right\|_{\left(H^{p}\left(H^{q}\right)\right)^{*}} .
$$

Estimate (6.194) and the disjointness of the dyadic intervals (see (J10) yield

$$
\sum_{K_{0}^{\prime}, L_{0}}\|T\|\left\|\sum_{K_{0}, L_{0}^{\prime}} \max _{K_{0}, L_{0}^{\prime}}\left(\|T\|\left|K_{0}\right|^{1 / p}\left|L_{0}\right|^{1 / q}\left|K_{0}^{\prime}\right|^{1 / p^{\prime}}\left|L_{0}^{\prime}\right|^{1 / q^{\prime}}\right) h_{K_{0} \times L_{0}^{\prime}}\right\|_{H^{p}\left(H^{q}\right)}\left\|h_{K_{0}^{\prime} \times L_{0}}\right\|_{\left(H^{p}\left(H^{q}\right)\right)^{*}} .
$$

Consequently, we obtain

$$
\sum_{K_{0}^{\prime}, L_{0}}\|T\| \max _{K_{0}, L_{0}^{\prime}}\left(\|T\|\left|K_{0}\right|^{1 / p}\left|L_{0}\right|^{1 / q}\left|K_{0}^{\prime}\right|^{1 / p^{\prime}}\left|L_{0}^{\prime}\right|^{1 / q^{\prime}}\right)\left\|\sum_{K_{0}, L_{0}^{\prime}} h_{K_{0} \times L_{0}^{\prime}}\right\|\left\|_{H^{p}\left(H^{q}\right)}\right\| h_{K_{0}^{\prime} \times L_{0}} \|_{\left(H^{p}\left(H^{q}\right)\right)^{*}} .
$$

Thus, (6.198) is bounded from above by

$$
\|T\|^{2} \max _{K_{0}, L_{0}^{\prime}}\left|K_{0}\right|^{1 / p}\left|L_{0}^{\prime}\right|^{1 / q^{\prime}} \sum_{K_{0}^{\prime}, L_{0}}\left|L_{0}\right|\left|K_{0}^{\prime}\right|^{2 / p^{\prime}}
$$

Using Hölder's inequality yields

$$
\|T\|^{2} \max _{K_{0}, K_{0}^{\prime}, L_{0}^{\prime}}\left|K_{0}\right|^{1 / p}\left|L_{0}^{\prime}\right|^{1 / q^{\prime}}\left|K_{0}^{\prime}\right|^{2 / p^{\prime}-1} .
$$

Inserting $\left|K_{0}\right|,\left|K_{0}^{\prime}\right|,\left|L_{0}^{\prime}\right| \leq \alpha$ (see (4.15)) into (6.199), we obtain the estimate

$$
\|T\|^{2} \alpha^{1 / p^{\prime}+1 / q^{\prime}} .
$$

CASE 8, GROUP (F2) : $K_{0}=K_{1} \neq K_{0}^{\prime}=K_{1}^{\prime}, L_{0}=L_{1}^{\prime} \neq L_{0}^{\prime}=L_{1}(((0)(1))$ (NIL NIL) $((1)(0)))$ - RIGHT VARIANT. In this case, we have to estimate

$$
\sum_{K_{0}, L_{0}^{\prime}, K_{0}^{\prime}, L_{0}}\left\langle T h_{K_{0} \times L_{0}}, h_{K_{0}^{\prime} \times L_{0}^{\prime}}\right\rangle\left\langle T h_{K_{0} \times L_{0}^{\prime}}, h_{K_{0}^{\prime} \times L_{0}}\right\rangle .
$$

We put $a_{K_{0}, K_{0}^{\prime}, L_{0}, L_{0}^{\prime}}=\left\langle T h_{K_{0} \times L_{0}}, h_{K_{0}^{\prime} \times L_{0}^{\prime}}\right\rangle$ and note the estimate

$$
\left|a_{K_{0}, K_{0}^{\prime}, L_{0}, L_{0}^{\prime}}\right| \leq \| T||\left|K_{0}\right|^{1 / p}\left|L_{0}\right|^{1 / q}\left|K_{0}^{\prime}\right|^{1 / p^{\prime}}\left|L_{0}^{\prime}\right|^{1 / q^{\prime}} .
$$


Now, we write 6.201) as follows:

$$
\sum_{K_{0}, L_{0}^{\prime}}\left\langle T h_{K_{0} \times L_{0}^{\prime}}, \sum_{K_{0}^{\prime}, L_{0}} a_{K_{0}, K_{0}^{\prime}, L_{0}, L_{0}^{\prime}} h_{K_{0}^{\prime} \times L_{0}}\right\rangle .
$$

By duality, we obtain the subsequent upper estimate for (6.203):

$$
\sum_{K_{0}, L_{0}^{\prime}}\left\|T h_{K_{0} \times L_{0}^{\prime}}\right\|_{H^{p}\left(H^{q}\right)}\left\|\sum_{K_{0}^{\prime}, L_{0}} a_{K_{0}, K_{0}^{\prime}, L_{0}, L_{0}^{\prime}} h_{K_{0}^{\prime} \times L_{0}}\right\|_{\left(H^{p}\left(H^{q}\right)\right)^{*}}
$$

Estimate (6.202) and the disjointness of the dyadic intervals (see (JJ1) yield

$$
\sum_{K_{0}, L_{0}^{\prime}}\|T\|\left\|h_{K_{0} \times L_{0}^{\prime}}\right\|_{H^{p}\left(H^{q}\right)}\left\|\sum_{K_{0}^{\prime}, L_{0}} \max _{K_{0}^{\prime}, L_{0}}\left(\|T\|\left|K_{0}\right|^{1 / p}\left|L_{0}\right|^{1 / q}\left|K_{0}^{\prime}\right|^{1 / p^{\prime}}\left|L_{0}^{\prime}\right|^{1 / q^{\prime}}\right) h_{K_{0}^{\prime} \times L_{0}}\right\|_{\left(H^{p}\left(H^{q}\right)\right)^{*}} .
$$

Consequently, we obtain

$$
\sum_{K_{0}, L_{0}^{\prime}}\|T\|\left\|h_{K_{0} \times L_{0}^{\prime}}\right\|_{H^{p}\left(H^{q}\right)} \max _{K_{0}^{\prime}, L_{0}}\left(\|T\|\left|K_{0}\right|^{1 / p}\left|L_{0}\right|^{1 / q}\left|K_{0}^{\prime}\right|^{1 / p^{\prime}}\left|L_{0}^{\prime}\right|^{1 / q^{\prime}}\right)\left\|\sum_{K_{0}^{\prime}, L_{0}} h_{K_{0}^{\prime} \times L_{0}}\right\|_{\left(H^{p}\left(H^{q}\right)\right)^{*}} .
$$

Thus, (6.206) is bounded from above by

$$
\|T\|^{2} \max _{K_{0}^{\prime}, L_{0}}\left|L_{0}\right|^{1 / q}\left|K_{0}^{\prime}\right|^{1 / p^{\prime}} \sum_{K_{0}, L_{0}^{\prime}}\left|K_{0}\right|^{2 / p}\left|L_{0}^{\prime}\right| .
$$

Using Hölder's inequality yields

$$
\|T\|^{2} \max _{K_{0}, K_{0}^{\prime}, L_{0}}\left|L_{0}\right|^{1 / q}\left|K_{0}^{\prime}\right|^{1 / p^{\prime}}\left|K_{0}\right|^{2 / p-1} .
$$

Inserting $\left|K_{0}\right|,\left|K_{0}^{\prime}\right|,\left|L_{0}\right| \leq \alpha$ (see (4.15)) into (6.207), we obtain the estimate

$$
\|T\|^{2} \alpha^{1 / p+1 / q} \text {. }
$$

Summary of Case 7 and Case 8. Combining (6.200) with (6.208) yields

$$
\mathbb{E}_{\theta, \varepsilon} Z^{2} \leq\|T\|^{2} \alpha
$$

CAse 9, Group ( $\mathrm{F3}): K_{0}=K_{1} \neq K_{0}^{\prime}=K_{1}^{\prime}, L_{0}=L_{0}^{\prime} \neq L_{1}=L_{1}^{\prime}(((1)(2))((0)$ (0)) (NIL NIL)) - LEFT VARIANT. In this case, we have to estimate

$$
\sum_{K_{0}^{\prime}, L_{1}, K_{0}, L_{0}}\left\langle T h_{K_{0} \times L_{0}}, h_{K_{0}^{\prime} \times L_{0}}\right\rangle\left\langle T h_{K_{0} \times L_{1}}, h_{K_{0}^{\prime} \times L_{1}}\right\rangle .
$$

We put $a_{K_{0}, K_{0}^{\prime}, L_{0}}=\left\langle T h_{K_{0} \times L_{0}}, h_{K_{0}^{\prime} \times L_{0}}\right\rangle$ and note the estimate

$$
\left|a_{K_{0}, K_{0}^{\prime}, L_{0}}\right| \leq\|T\|\left|K_{0}\right|^{1 / p}\left|L_{0}\right|^{1 / q}\left|K_{0}^{\prime}\right|^{1 / p^{\prime}}\left|L_{0}\right|^{1 / q^{\prime}} .
$$

Now, we write (6.210) as follows:

$$
\sum_{K_{0}^{\prime}, L_{1}}\left\langle T \sum_{K_{0}, L_{0}} a_{K_{0}, K_{0}^{\prime}, L_{0}} h_{K_{0} \times L_{1}}, h_{K_{0}^{\prime} \times L_{1}}\right\rangle .
$$

By duality, we obtain the subsequent upper estimate for (6.212):

$$
\sum_{K_{0}^{\prime}, L_{1}}\left\|T \sum_{K_{0}, L_{0}} a_{K_{0}, K_{0}^{\prime}, L_{0}} h_{K_{0} \times L_{1}}\right\|_{H^{p}\left(H^{q}\right)}\left\|h_{K_{0}^{\prime} \times L_{1}}\right\|_{\left(H^{p}\left(H^{q}\right)\right)^{*}} \cdot
$$


Estimate (6.211) and the disjointness of the dyadic intervals (see (J1)) yield

$$
\sum_{K_{0}^{\prime}, L_{1}}\|T\|\left\|\sum_{K_{0}, L_{0}} \max _{K_{0}}\left(\|T\|\left|K_{0}\right|^{1 / p}\left|L_{0}\right|^{1 / q}\left|K_{0}^{\prime}\right|^{1 / p^{\prime}}\left|L_{0}\right|^{1 / q^{\prime}}\right) h_{K_{0} \times L_{1}}\right\|_{H^{p}\left(H^{q}\right)}\left\|h_{K_{0}^{\prime} \times L_{1}}\right\|_{\left(H^{p}\left(H^{q}\right)\right)^{*}} \text {. }
$$

Consequently, we obtain

$$
\sum_{K_{0}^{\prime}, L_{1}}\|T\| \sum_{L_{0}} \max _{K_{0}}\left(\|T\|\left|K_{0}\right|^{1 / p}\left|L_{0}\right|^{1 / q}\left|K_{0}^{\prime}\right|^{1 / p^{\prime}}\left|L_{0}\right|^{1 / q^{\prime}}\right)\left\|\sum_{K_{0}} h_{K_{0} \times L_{1}}\right\|_{H^{p}\left(H^{q}\right)}\left\|h_{K_{0}^{\prime} \times L_{1}}\right\|_{\left(H^{p}\left(H^{q}\right)\right)^{*}} \text {. }
$$

Thus, 6.215) is bounded from above by

$$
\|T\|^{2} \max _{K_{0}}\left|K_{0}\right|^{1 / p} \sum_{K_{0}^{\prime}, L_{1}} \sum_{L_{0}}\left|L_{0}\right|\left|K_{0}^{\prime}\right|^{2 / p^{\prime}}\left|L_{1}\right| .
$$

Using Hölder's inequality yields

$$
\|T\|^{2} \max _{K_{0}, K_{0}^{\prime}}\left|K_{0}\right|^{1 / p}\left|K_{0}^{\prime}\right|^{2 / p^{\prime}-1} .
$$

Inserting $\left|K_{0}\right|,\left|K_{0}^{\prime}\right| \leq \alpha$ (see (4.15) ) into (6.216), we obtain the estimate

$$
\|T\|^{2} \alpha^{1 / p^{\prime}}
$$

CAse 10, Group (F3): $K_{0}=K_{1} \neq K_{0}^{\prime}=K_{1}^{\prime}, L_{0}=L_{0}^{\prime} \neq L_{1}=L_{1}^{\prime}(((0)(2))$ (NIL NIL) $((1)(0)))$ - RIGHT VARIANT. In this case, we have to estimate

$$
\sum_{K_{0}, L_{1}, K_{0}^{\prime}, L_{0}}\left\langle T h_{K_{0} \times L_{0}}, h_{K_{0}^{\prime} \times L_{0}}\right\rangle\left\langle T h_{K_{0} \times L_{1}}, h_{K_{0}^{\prime} \times L_{1}}\right\rangle .
$$

We put $a_{K_{0}, K_{0}^{\prime}, L_{0}}=\left\langle T h_{K_{0} \times L_{0}}, h_{K_{0}^{\prime} \times L_{0}}\right\rangle$ and note the estimate

$$
\left|a_{K_{0}, K_{0}^{\prime}, L_{0}}\right| \leq\|T\|\left|K_{0}\right|^{1 / p}\left|L_{0}\right|^{1 / q}\left|K_{0}^{\prime}\right|^{1 / p^{\prime}}\left|L_{0}\right|^{1 / q^{\prime}} .
$$

Now, we write (6.218) as follows:

$$
\sum_{K_{0}, L_{1}}\left\langle T h_{K_{0} \times L_{1}}, \sum_{K_{0}^{\prime}, L_{0}} a_{K_{0}, K_{0}^{\prime}, L_{0}} h_{K_{0}^{\prime} \times L_{1}}\right\rangle .
$$

By duality, we obtain the subsequent upper estimate for (6.220):

$$
\sum_{K_{0}, L_{1}}\left\|T h_{K_{0} \times L_{1}}\right\|_{H^{p}\left(H^{q}\right)}\left\|\sum_{K_{0}^{\prime}, L_{0}} a_{K_{0}, K_{0}^{\prime}, L_{0}} h_{K_{0}^{\prime} \times L_{1}}\right\|_{\left(H^{p}\left(H^{q}\right)\right)^{*}} .
$$

Estimate (6.219) and the disjointness of the dyadic intervals (see (J1)) yield

$$
\sum_{K_{0}, L_{1}}\|T\|\left\|h_{K_{0} \times L_{1}}\right\|_{H^{p}\left(H^{q}\right)}\left\|\sum_{K_{0}^{\prime}, L_{0}} \max _{K_{0}^{\prime}}\left(\|T\|\left|K_{0}\right|^{1 / p}\left|L_{0}\right|^{1 / q}\left|K_{0}^{\prime}\right|^{1 / p^{\prime}}\left|L_{0}\right|^{1 / q^{\prime}}\right) h_{K_{0}^{\prime} \times L_{1}}\right\|_{\left(H^{p}\left(H^{q}\right)\right)^{*}} .
$$

Consequently, we obtain

$$
\sum_{K_{0}, L_{1}}\|T\|\left\|h_{K_{0} \times L_{1}}\right\|_{H^{p}\left(H^{q}\right)} \sum_{L_{0}} \max _{K_{0}^{\prime}}\left(\|T\|\left|K_{0}\right|^{1 / p}\left|L_{0}\right|^{1 / q}\left|K_{0}^{\prime}\right|^{1 / p^{\prime}}\left|L_{0}\right|^{1 / q^{\prime}}\right)\left\|\sum_{K_{0}^{\prime}} h_{K_{0}^{\prime} \times L_{1}}\right\|_{\left(H^{p}\left(H^{q}\right)\right)^{*}} .
$$

Thus, 6.223) is bounded from above by

$$
\|T\|^{2} \max _{K_{0}^{\prime}}\left|K_{0}^{\prime}\right|^{1 / p^{\prime}} \sum_{K_{0}, L_{1}} \sum_{L_{0}}\left|K_{0}\right|^{2 / p}\left|L_{0}\right|\left|L_{1}\right| .
$$


DIMENSION DEPENDENCE OF FACTORIZATION PROBLEMS: BI-PARAMETER HARDY SPACBS

Using Hölder's inequality yields

$$
\|T\|^{2} \max _{K_{0}, K_{0}^{\prime}}\left|K_{0}^{\prime}\right|^{1 / p^{\prime}}\left|K_{0}\right|^{2 / p-1} .
$$

Inserting $\left|K_{0}\right|,\left|K_{0}^{\prime}\right| \leq \alpha$ (see (4.15) ) into (6.224), we obtain the estimate

$$
\|T\|^{2} \alpha^{1 / p}
$$

Summary of Case 9 and Case 10. Combining (6.217) with (6.225) yields

$$
\mathbb{E}_{\theta, \varepsilon} Z^{2} \leq\|T\|^{2} \alpha^{1 / 2}
$$

CASE 11, Group (F4): $K_{0}=K_{1}^{\prime} \neq K_{0}^{\prime}=K_{1}, L_{0}=L_{1} \neq L_{0}^{\prime}=L_{1}^{\prime}(((0)(1))((1)$ (0)) (NIL NIL)) - LEFT VARIANT. In this case, we have to estimate

$$
\sum_{K_{0}, L_{0}^{\prime}, K_{0}^{\prime}, L_{0}}\left\langle T h_{K_{0} \times L_{0}}, h_{K_{0}^{\prime} \times L_{0}^{\prime}}\right\rangle\left\langle T h_{K_{0}^{\prime} \times L_{0}}, h_{K_{0} \times L_{0}^{\prime}}\right\rangle .
$$

We put $a_{K_{0}, K_{0}^{\prime}, L_{0}, L_{0}^{\prime}}=\left\langle T h_{K_{0} \times L_{0}}, h_{K_{0}^{\prime} \times L_{0}^{\prime}}\right\rangle$ and note the estimate

$$
\left|a_{K_{0}, K_{0}^{\prime}, L_{0}, L_{0}^{\prime}}\right| \leq\|T\|\left|K_{0}\right|^{1 / p}\left|L_{0}\right|^{1 / q}\left|K_{0}^{\prime}\right|^{1 / p^{\prime}}\left|L_{0}^{\prime}\right|^{1 / q^{\prime}}
$$

Now, we write 6.227) as follows:

$$
\sum_{K_{0}, L_{0}^{\prime}}\left\langle T \sum_{K_{0}^{\prime}, L_{0}} a_{K_{0}, K_{0}^{\prime}, L_{0}, L_{0}^{\prime}} h_{K_{0}^{\prime} \times L_{0}}, h_{K_{0} \times L_{0}^{\prime}}\right\rangle .
$$

By duality, we obtain the subsequent upper estimate for 6.229):

$$
\sum_{K_{0}, L_{0}^{\prime}}\left\|T \sum_{K_{0}^{\prime}, L_{0}} a_{K_{0}, K_{0}^{\prime}, L_{0}, L_{0}^{\prime}} h_{K_{0}^{\prime} \times L_{0}}\right\|_{H^{p}\left(H^{q}\right)}\left\|h_{K_{0} \times L_{0}^{\prime}}\right\|_{\left(H^{p}\left(H^{q}\right)\right)^{*}} \cdot
$$

Estimate (6.228) and the disjointness of the dyadic intervals (see (J1)) yield

$$
\sum_{K_{0}, L_{0}^{\prime}}\|T\|\left\|\sum_{K_{0}^{\prime}, L_{0}} \max _{K_{0}^{\prime}, L_{0}}\left(\|T\|\left|K_{0}\right|^{1 / p}\left|L_{0}\right|^{1 / q}\left|K_{0}^{\prime}\right|^{1 / p^{\prime}}\left|L_{0}^{\prime}\right|^{1 / q^{\prime}}\right) h_{K_{0}^{\prime} \times L_{0}}\right\|_{H^{p}\left(H^{q}\right)}\left\|h_{K_{0} \times L_{0}^{\prime}}\right\|_{\left(H^{p}\left(H^{q}\right)\right)^{*}} .
$$

Consequently, we obtain

$$
\sum_{K_{0}, L_{0}^{\prime}}\|T\| \max _{K_{0}^{\prime}, L_{0}}\left(\|T\|\left|K_{0}\right|^{1 / p}\left|L_{0}\right|^{1 / q}\left|K_{0}^{\prime}\right|^{1 / p^{\prime}}\left|L_{0}^{\prime}\right|^{1 / q^{\prime}}\right)\left\|\sum_{K_{0}^{\prime}, L_{0}} h_{K_{0}^{\prime} \times L_{0}}\right\|_{H^{p}\left(H^{q}\right)}\left\|h_{K_{0} \times L_{0}^{\prime}}\right\|_{\left(H^{p}\left(H^{q}\right)\right)^{*}} .
$$

Thus, (6.232) is bounded from above by

$$
\|T\|^{2} \max _{K_{0}^{\prime}, L_{0}}\left|L_{0}\right|^{1 / q}\left|K_{0}^{\prime}\right|^{1 / p^{\prime}} \sum_{K_{0}, L_{0}^{\prime}}\left|K_{0}\right|\left|L_{0}^{\prime}\right|^{2 / q^{\prime}} .
$$

Using Hölder's inequality yields

$$
\|T\|^{2} \max _{K_{0}^{\prime}, L_{0}, L_{0}^{\prime}}\left|L_{0}\right|^{1 / q}\left|K_{0}^{\prime}\right|^{1 / p^{\prime}}\left|L_{0}^{\prime}\right|^{2 / q^{\prime}-1} .
$$

Inserting $\left|K_{0}^{\prime}\right|,\left|L_{0}\right|,\left|L_{0}^{\prime}\right| \leq \alpha$ (see (4.15)) into (6.233), we obtain the estimate

$$
\|T\|^{2} \alpha^{1 / p^{\prime}+1 / q^{\prime}}
$$

CAse 12, Group (F4): $K_{0}=K_{1}^{\prime} \neq K_{0}^{\prime}=K_{1}, L_{0}=L_{1} \neq L_{0}^{\prime}=L_{1}^{\prime}(((1)(0))$ (NIL NIL) $((0)(1)))$ - RIGHT VARIANT. In this case, we have to estimate

$$
\sum_{K_{0}^{\prime}, L_{0}, K_{0}, L_{0}^{\prime}}\left\langle T h_{K_{0} \times L_{0}}, h_{K_{0}^{\prime} \times L_{0}^{\prime}}\right\rangle\left\langle T h_{K_{0}^{\prime} \times L_{0}}, h_{K_{0} \times L_{0}^{\prime}}\right\rangle .
$$


We put $a_{K_{0}, K_{0}^{\prime}, L_{0}, L_{0}^{\prime}}=\left\langle T h_{K_{0} \times L_{0}}, h_{K_{0}^{\prime} \times L_{0}^{\prime}}\right\rangle$ and note the estimate

$$
\left|a_{K_{0}, K_{0}^{\prime}, L_{0}, L_{0}^{\prime}}\right| \leq\|T\|\left|K_{0}\right|^{1 / p}\left|L_{0}\right|^{1 / q}\left|K_{0}^{\prime}\right|^{1 / p^{\prime}}\left|L_{0}^{\prime}\right|^{1 / q^{\prime}} .
$$

Now, we write (6.235) as follows:

$$
\sum_{K_{0}^{\prime}, L_{0}}\left\langle T h_{K_{0}^{\prime} \times L_{0}}, \sum_{K_{0}, L_{0}^{\prime}} a_{K_{0}, K_{0}^{\prime}, L_{0}, L_{0}^{\prime}} h_{K_{0} \times L_{0}^{\prime}}\right\rangle .
$$

By duality, we obtain the subsequent upper estimate for (6.237):

$$
\sum_{K_{0}^{\prime}, L_{0}}\left\|T h_{K_{0}^{\prime} \times L_{0}}\right\|_{H^{p}\left(H^{q}\right)}\left\|\sum_{K_{0}, L_{0}^{\prime}} a_{K_{0}, K_{0}^{\prime}, L_{0}, L_{0}^{\prime}} h_{K_{0} \times L_{0}^{\prime}}\right\|_{\left(H^{p}\left(H^{q}\right)\right)^{*}} .
$$

Estimate (6.236) and the disjointness of the dyadic intervals (see (J1)) yield

$$
\sum_{K_{0}^{\prime}, L_{0}}\|T\|\left\|h_{K_{0}^{\prime} \times L_{0}}\right\|_{H^{p}\left(H^{q}\right)}\left\|\sum_{K_{0}, L_{0}^{\prime}} \max _{K_{0}, L_{0}^{\prime}}\left(\|T\|\left|K_{0}\right|^{1 / p}\left|L_{0}\right|^{1 / q}\left|K_{0}^{\prime}\right|^{1 / p^{\prime}}\left|L_{0}^{\prime}\right|^{1 / q^{\prime}}\right) h_{K_{0} \times L_{0}^{\prime}}\right\|_{\left(H^{p}\left(H^{q}\right)\right)^{*}} .
$$

Consequently, we obtain

$$
\sum_{K_{0}^{\prime}, L_{0}}\|T\|\left\|h_{K_{0}^{\prime} \times L_{0}}\right\|_{H^{p}\left(H^{q}\right)} \max _{K_{0}, L_{0}^{\prime}}\left(\|T\|\left|K_{0}\right|^{1 / p}\left|L_{0}\right|^{1 / q}\left|K_{0}^{\prime}\right|^{1 / p^{\prime}}\left|L_{0}^{\prime}\right|^{1 / q^{\prime}}\right)\left\|\sum_{K_{0}, L_{0}^{\prime}} h_{K_{0} \times L_{0}^{\prime}}\right\|_{\left(H^{p}\left(H^{q}\right)\right)^{*}} .
$$

Thus, (6.240) is bounded from above by

$$
\|T\|^{2} \max _{K_{0}, L_{0}^{\prime}}\left|K_{0}\right|^{1 / p}\left|L_{0}^{\prime}\right|^{1 / q^{\prime}} \sum_{K_{0}^{\prime}, L_{0}}\left|L_{0}\right|^{2 / q}\left|K_{0}^{\prime}\right| .
$$

Using Hölder's inequality yields

$$
\|T\|^{2} \max _{K_{0}, L_{0}, L_{0}^{\prime}}\left|K_{0}\right|^{1 / p}\left|L_{0}^{\prime}\right|^{1 / q^{\prime}}\left|L_{0}\right|^{2 / q-1} .
$$

Inserting $\left|K_{0}\right|,\left|L_{0}\right|,\left|L_{0}^{\prime}\right| \leq \alpha$ (see (4.15)) into (6.241), we obtain the estimate

$$
\|T\|^{2} \alpha^{1 / p+1 / q} .
$$

Summary of Case 11 and Case 12. Combining (6.234) with (6.242) yields

$$
\mathbb{E}_{\theta, \varepsilon} Z^{2} \leq\|T\|^{2} \alpha .
$$

CASE 13, Group (F5) : $K_{0}=K_{1}^{\prime} \neq K_{0}^{\prime}=K_{1}, L_{0}=L_{1}^{\prime} \neq L_{0}^{\prime}=L_{1}(((0)(0))((1)$

(1)) (NIL NIL)) - LEFT VARIANT. In this case, we have to estimate

$$
\sum_{K_{0}, L_{0}, K_{0}^{\prime}, L_{0}^{\prime}}\left\langle T h_{K_{0} \times L_{0}}, h_{K_{0}^{\prime} \times L_{0}^{\prime}}\right\rangle\left\langle T h_{K_{0}^{\prime} \times L_{0}^{\prime}}, h_{K_{0} \times L_{0}}\right\rangle .
$$

We put $a_{K_{0}, K_{0}^{\prime}, L_{0}, L_{0}^{\prime}}=\left\langle T h_{K_{0} \times L_{0}}, h_{K_{0}^{\prime} \times L_{0}^{\prime}}\right\rangle$ and note the estimate

$$
\left|a_{K_{0}, K_{0}^{\prime}, L_{0}, L_{0}^{\prime}}\right| \leq\|T\|\left|K_{0}\right|^{1 / p}\left|L_{0}\right|^{1 / q}\left|K_{0}^{\prime}\right|^{1 / p^{\prime}}\left|L_{0}^{\prime}\right|^{1 / q^{\prime}} .
$$

Now, we write (6.244) as follows:

$$
\sum_{K_{0}, L_{0}}\left\langle T \sum_{K_{0}^{\prime}, L_{0}^{\prime}} a_{K_{0}, K_{0}^{\prime}, L_{0}, L_{0}^{\prime}} h_{K_{0}^{\prime} \times L_{0}^{\prime}}, h_{K_{0} \times L_{0}}\right\rangle .
$$

By duality, we obtain the subsequent upper estimate for (6.246):

$$
\sum_{K_{0}, L_{0}}\left\|T \sum_{K_{0}^{\prime}, L_{0}^{\prime}} a_{K_{0}, K_{0}^{\prime}, L_{0}, L_{0}^{\prime}} h_{K_{0}^{\prime} \times L_{0}^{\prime}}\right\|_{H^{p}\left(H^{q}\right)}\left\|h_{K_{0} \times L_{0}}\right\|_{\left(H^{p}\left(H^{q}\right)\right)^{*}} .
$$


DIMENSION DEPENDENCE OF FACTORIZATION PROBLEMS: BI-PARAMETER HARDY SPACBS

Estimate (6.245) and the disjointness of the dyadic intervals (see (J1)) yield

$\sum_{K_{0}, L_{0}}\|T\|\left\|\sum_{K_{0}^{\prime}, L_{0}^{\prime}} \max _{K_{0}^{\prime}, L_{0}^{\prime}}\left(\|T\|\left|K_{0}\right|^{1 / p}\left|L_{0}\right|^{1 / q}\left|K_{0}^{\prime}\right|^{1 / p^{\prime}}\left|L_{0}^{\prime}\right|^{1 / q^{\prime}}\right) h_{K_{0}^{\prime} \times L_{0}^{\prime}}\right\|_{H^{p}\left(H^{q}\right)}\left\|h_{K_{0} \times L_{0}}\right\|_{\left(H^{p}\left(H^{q}\right)\right)^{*}}$.

Consequently, we obtain

$\sum_{K_{0}, L_{0}}\|T\| \max _{K_{0}^{\prime}, L_{0}^{\prime}}\left(\|T\|\left|K_{0}\right|^{1 / p}\left|L_{0}\right|^{1 / q}\left|K_{0}^{\prime}\right|^{1 / p^{\prime}}\left|L_{0}^{\prime}\right|^{1 / q^{\prime}}\right)\left\|\sum_{K_{0}^{\prime}, L_{0}^{\prime}} h_{K_{0}^{\prime} \times L_{0}^{\prime}}\right\|\left\|_{H^{p}\left(H^{q}\right)}\right\| h_{K_{0} \times L_{0}} \|_{\left(H^{p}\left(H^{q}\right)\right)^{*}}$.

Thus, (6.249) is bounded from above by

$$
\|T\|^{2} \max _{K_{0}^{\prime}, L_{0}^{\prime}}\left|K_{0}^{\prime}\right|^{1 / p^{\prime}}\left|L_{0}^{\prime}\right|^{1 / q^{\prime}} \sum_{K_{0}, L_{0}}\left|K_{0}\right|\left|L_{0}\right| \leq\|T\|^{2} \max _{K_{0}^{\prime}, L_{0}^{\prime}}\left|K_{0}^{\prime}\right|^{1 / p^{\prime}}\left|L_{0}^{\prime}\right|^{1 / q^{\prime}} .
$$

Inserting $\left|K_{0}^{\prime}\right|,\left|L_{0}^{\prime}\right| \leq \alpha$ (see (4.15) ) into (6.250), we obtain the estimate

$$
\|T\|^{2} \alpha^{1 / p^{\prime}+1 / q^{\prime}}
$$

CASE 14, GROUP (F5): $K_{0}=K_{1}^{\prime} \neq K_{0}^{\prime}=K_{1}, L_{0}=L_{1}^{\prime} \neq L_{0}^{\prime}=L_{1}(((1)(1))$ (NIL NIL) $((0)(0)))$ - RIGHT VARIANT. In this case, we have to estimate

$$
\sum_{K_{0}^{\prime}, L_{0}^{\prime}, K_{0}, L_{0}}\left\langle T h_{K_{0} \times L_{0}}, h_{K_{0}^{\prime} \times L_{0}^{\prime}}\right\rangle\left\langle T h_{K_{0}^{\prime} \times L_{0}^{\prime}}, h_{K_{0} \times L_{0}}\right\rangle .
$$

We put $a_{K_{0}, K_{0}^{\prime}, L_{0}, L_{0}^{\prime}}=\left\langle T h_{K_{0} \times L_{0}}, h_{K_{0}^{\prime} \times L_{0}^{\prime}}\right\rangle$ and note the estimate

$$
\left|a_{K_{0}, K_{0}^{\prime}, L_{0}, L_{0}^{\prime}}\right| \leq \| T||\left|K_{0}\right|^{1 / p}\left|L_{0}\right|^{1 / q}\left|K_{0}^{\prime}\right|^{1 / p^{\prime}}\left|L_{0}^{\prime}\right|^{1 / q^{\prime}} .
$$

Now, we write (6.252) as follows:

$$
\sum_{K_{0}^{\prime}, L_{0}^{\prime}}\left\langle T h_{K_{0}^{\prime} \times L_{0}^{\prime}}, \sum_{K_{0}, L_{0}} a_{K_{0}, K_{0}^{\prime}, L_{0}, L_{0}^{\prime}} h_{K_{0} \times L_{0}}\right\rangle .
$$

By duality, we obtain the subsequent upper estimate for (6.254):

$$
\sum_{K_{0}^{\prime}, L_{0}^{\prime}}\left\|T h_{K_{0}^{\prime} \times L_{0}^{\prime}}\right\|_{H^{p}\left(H^{q}\right)}\left\|\sum_{K_{0}, L_{0}} a_{K_{0}, K_{0}^{\prime}, L_{0}, L_{0}^{\prime}} h_{K_{0} \times L_{0}}\right\|_{\left(H^{p}\left(H^{q}\right)\right)^{*}} .
$$

Estimate (6.253) and the disjointness of the dyadic intervals (see (JJ1) yield

$$
\sum_{K_{0}^{\prime}, L_{0}^{\prime}}\|T\|\left\|h_{K_{0}^{\prime} \times L_{0}^{\prime}}\right\|_{H^{p}\left(H^{q}\right)}\left\|\sum_{K_{0}, L_{0}} \max _{K_{0}, L_{0}}\left(\|T\|\left|K_{0}\right|^{1 / p}\left|L_{0}\right|^{1 / q}\left|K_{0}^{\prime}\right|^{1 / p^{\prime}}\left|L_{0}^{\prime}\right|^{1 / q^{\prime}}\right) h_{K_{0} \times L_{0}}\right\|_{\left(H^{p}\left(H^{q}\right)\right)^{*}} .
$$

Consequently, we obtain

$$
\sum_{K_{0}^{\prime}, L_{0}^{\prime}}\|T\|\left\|h_{K_{0}^{\prime} \times L_{0}^{\prime}}\right\|_{H^{p}\left(H^{q}\right)} \max _{K_{0}, L_{0}}\left(\|T\|\left|K_{0}\right|^{1 / p}\left|L_{0}\right|^{1 / q}\left|K_{0}^{\prime}\right|^{1 / p^{\prime}}\left|L_{0}^{\prime}\right|^{1 / q^{\prime}}\right)\left\|\sum_{K_{0}, L_{0}} h_{K_{0} \times L_{0}}\right\|_{\left(H^{p}\left(H^{q}\right)\right)^{*}} .
$$

Thus, (6.257) is bounded from above by

$$
\|T\|^{2} \max _{K_{0}, L_{0}}\left|K_{0}\right|^{1 / p}\left|L_{0}\right|^{1 / q} \sum_{K_{0}^{\prime}, L_{0}^{\prime}}\left|K_{0}^{\prime}\right|\left|L_{0}^{\prime}\right| \leq\|T\|^{2} \max _{K_{0}, L_{0}}\left|K_{0}\right|^{1 / p}\left|L_{0}\right|^{1 / q} .
$$

Inserting $\left|K_{0}\right|,\left|L_{0}\right| \leq \alpha$ (see (4.15)) into (6.258), we obtain the estimate

$$
\|T\|^{2} \alpha^{1 / p+1 / q} \text {. }
$$


Summary of Case 13 and Case 14. Combining (6.251) with (6.259) yields

$$
\mathbb{E}_{\theta, \varepsilon} Z^{2} \leq\|T\|^{2} \alpha \text {. }
$$

CAse 15, Group (F6) $: K_{0}=K_{1}^{\prime} \neq K_{0}^{\prime}=K_{1}, L_{0}=L_{0}^{\prime} \neq L_{1}=L_{1}^{\prime}(((0)(2))((1)$ (0)) (NIL NIL)) - LEFT VARIANT. In this case, we have to estimate

$$
\sum_{K_{0}, L_{1}, K_{0}^{\prime}, L_{0}}\left\langle T h_{K_{0} \times L_{0}}, h_{K_{0}^{\prime} \times L_{0}}\right\rangle\left\langle T h_{K_{0}^{\prime} \times L_{1}}, h_{K_{0} \times L_{1}}\right\rangle .
$$

We put $a_{K_{0}, K_{0}^{\prime}, L_{0}}=\left\langle T h_{K_{0} \times L_{0}}, h_{K_{0}^{\prime} \times L_{0}}\right\rangle$ and note the estimate

$$
\left|a_{K_{0}, K_{0}^{\prime}, L_{0}}\right| \leq\|T\|\left|K_{0}\right|^{1 / p}\left|L_{0}\right|^{1 / q}\left|K_{0}^{\prime}\right|^{1 / p^{\prime}}\left|L_{0}\right|^{1 / q^{\prime}} .
$$

Now, we write 6.261) as follows:

$$
\sum_{K_{0}, L_{1}}\left\langle T \sum_{K_{0}^{\prime}, L_{0}} a_{K_{0}, K_{0}^{\prime}, L_{0}} h_{K_{0}^{\prime} \times L_{1}}, h_{K_{0} \times L_{1}}\right\rangle .
$$

By duality, we obtain the subsequent upper estimate for (6.263):

$$
\sum_{K_{0}, L_{1}}\left\|T \sum_{K_{0}^{\prime}, L_{0}} a_{K_{0}, K_{0}^{\prime}, L_{0}} h_{K_{0}^{\prime} \times L_{1}}\right\|_{H^{p}\left(H^{q}\right)}\left\|h_{K_{0} \times L_{1}}\right\|_{\left(H^{p}\left(H^{q}\right)\right)^{*}} .
$$

Estimate (6.262) and the disjointness of the dyadic intervals (see (JJ1) yield

$$
\sum_{K_{0}, L_{1}}\|T\|\left\|\sum_{K_{0}^{\prime}, L_{0}} \max _{K_{0}^{\prime}}\left(\|T\|\left|K_{0}\right|^{1 / p}\left|L_{0}\right|^{1 / q}\left|K_{0}^{\prime}\right|^{1 / p^{\prime}}\left|L_{0}\right|^{1 / q^{\prime}}\right) h_{K_{0}^{\prime} \times L_{1}}\right\|_{H^{p}\left(H^{q}\right)}\left\|h_{K_{0} \times L_{1}}\right\|_{\left(H^{p}\left(H^{q}\right)\right)^{*}} .
$$

Consequently, we obtain

$$
\sum_{K_{0}, L_{1}}\|T\| \sum_{L_{0}} \max _{K_{0}^{\prime}}\left(\|T\|\left|K_{0}\right|^{1 / p}\left|L_{0}\right|^{1 / q}\left|K_{0}^{\prime}\right|^{1 / p^{\prime}}\left|L_{0}\right|^{1 / q^{\prime}}\right)\left\|\sum_{K_{0}^{\prime}} h_{K_{0}^{\prime} \times L_{1}}\right\|_{H^{p}\left(H^{q}\right)}\left\|h_{K_{0} \times L_{1}}\right\|_{\left(H^{p}\left(H^{q}\right)\right)^{*}} .
$$

Thus, 6.266) is bounded from above by

$$
\|T\|^{2} \max _{K_{0}^{\prime}}\left|K_{0}^{\prime}\right|^{1 / p^{\prime}} \sum_{K_{0}, L_{1}} \sum_{L_{0}}\left|K_{0}\right|\left|L_{0}\right|\left|L_{1}\right| \leq\|T\|^{2} \max _{K_{0}^{\prime}}\left|K_{0}^{\prime}\right|^{1 / p^{\prime}} .
$$

Inserting $\left|K_{0}^{\prime}\right| \leq \alpha$ (see (4.15)) into (6.267), we obtain the estimate

$$
\|T\|^{2} \alpha^{1 / p^{\prime}} \text {. }
$$

CAse 16, GRoup (F6): $K_{0}=K_{1}^{\prime} \neq K_{0}^{\prime}=K_{1}, L_{0}=L_{0}^{\prime} \neq L_{1}=L_{1}^{\prime}(((1)(2))$ (NIL NIL) $((0)(0)))$ - RIGHT VARIANT. In this case, we have to estimate

$$
\sum_{K_{0}^{\prime}, L_{1}, K_{0}, L_{0}}\left\langle T h_{K_{0} \times L_{0}}, h_{K_{0}^{\prime} \times L_{0}}\right\rangle\left\langle T h_{K_{0}^{\prime} \times L_{1}}, h_{K_{0} \times L_{1}}\right\rangle .
$$

We put $a_{K_{0}, K_{0}^{\prime}, L_{0}}=\left\langle T h_{K_{0} \times L_{0}}, h_{K_{0}^{\prime} \times L_{0}}\right\rangle$ and note the estimate

$$
\left|a_{K_{0}, K_{0}^{\prime}, L_{0}}\right| \leq\|T\|\left|K_{0}\right|^{1 / p}\left|L_{0}\right|^{1 / q}\left|K_{0}^{\prime}\right|^{1 / p^{\prime}}\left|L_{0}\right|^{1 / q^{\prime}} .
$$

Now, we write (6.269) as follows:

$$
\sum_{K_{0}^{\prime}, L_{1}}\left\langle T h_{K_{0}^{\prime} \times L_{1}}, \sum_{K_{0}, L_{0}} a_{K_{0}, K_{0}^{\prime}, L_{0}} h_{K_{0} \times L_{1}}\right\rangle .
$$


DIMENSION DEPENDENCE OF FACTORIZATION PROBLEMS: BI-PARAMETER HARDY SPACB\$

By duality, we obtain the subsequent upper estimate for (6.271):

$$
\sum_{K_{0}^{\prime}, L_{1}}\left\|T h_{K_{0}^{\prime} \times L_{1}}\right\|_{H^{p}\left(H^{q}\right)}\left\|\sum_{K_{0}, L_{0}} a_{K_{0}, K_{0}^{\prime}, L_{0}} h_{K_{0} \times L_{1}}\right\|_{\left(H^{p}\left(H^{q}\right)\right)^{*}} .
$$

Estimate (6.270) and the disjointness of the dyadic intervals (see (JJ1) yield

$$
\sum_{K_{0}^{\prime}, L_{1}}\|T\|\left\|h_{K_{0}^{\prime} \times L_{1}}\right\|_{H^{p}\left(H^{q}\right)}\left\|\sum_{K_{0}, L_{0}} \max _{K_{0}}\left(\|T\|\left|K_{0}\right|^{1 / p}\left|L_{0}\right|^{1 / q}\left|K_{0}^{\prime}\right|^{1 / p^{\prime}}\left|L_{0}\right|^{1 / q^{\prime}}\right) h_{K_{0} \times L_{1}}\right\|_{\left(H^{p}\left(H^{q}\right)\right)^{*}} .
$$

Consequently, we obtain

$$
\sum_{K_{0}^{\prime}, L_{1}}\|T\|\left\|h_{K_{0}^{\prime} \times L_{1}}\right\|_{H^{p}\left(H^{q}\right)} \sum_{L_{0}} \max _{K_{0}}\left(\|T\|\left|K_{0}\right|^{1 / p}\left|L_{0}\right|^{1 / q}\left|K_{0}^{\prime}\right|^{1 / p^{\prime}}\left|L_{0}\right|^{1 / q^{\prime}}\right)\left\|\sum_{K_{0}} h_{K_{0} \times L_{1}}\right\|_{\left(H^{p}\left(H^{q}\right)\right)^{*}} .
$$

Thus, (6.274) is bounded from above by

$$
\|T\|^{2} \max _{K_{0}}\left|K_{0}\right|^{1 / p} \sum_{K_{0}^{\prime}, L_{1}} \sum_{L_{0}}\left|L_{0}\right|\left|K_{0}^{\prime}\right|\left|L_{1}\right| \leq\|T\|^{2} \max _{K_{0}}\left|K_{0}\right|^{1 / p} .
$$

Inserting $\left|K_{0}\right| \leq \alpha$ (see (4.15) ) into (6.275), we obtain the estimate

$$
\|T\|^{2} \alpha^{1 / p} \text {. }
$$

Summary of Case 15 and Case 16. Combining (6.268) with (6.276) yields

$$
\mathbb{E}_{\theta, \varepsilon} Z^{2} \leq\|T\|^{2} \alpha^{1 / 2}
$$

CASE 17, Group (F7) : $K_{0}=K_{0}^{\prime} \neq K_{1}=K_{1}^{\prime}, L_{0}=L_{1} \neq L_{0}^{\prime}=L_{1}^{\prime}(((2)(1))((0)$ (0)) (NIL NIL)) - LEFT VARIANT. In this case, we have to estimate

$$
\sum_{K_{1}, L_{0}^{\prime}, K_{0}, L_{0}}\left\langle T h_{K_{0} \times L_{0}}, h_{K_{0} \times L_{0}^{\prime}}\right\rangle\left\langle T h_{K_{1} \times L_{0}}, h_{K_{1} \times L_{0}^{\prime}}\right\rangle .
$$

We put $a_{K_{0}, L_{0}, L_{0}^{\prime}}=\left\langle T h_{K_{0} \times L_{0}}, h_{K_{0} \times L_{0}^{\prime}}\right\rangle$ and note the estimate

$$
\left|a_{K_{0}, L_{0}, L_{0}^{\prime}}\right| \leq\|T\|\left|K_{0}\right|^{1 / p}\left|L_{0}\right|^{1 / q}\left|K_{0}\right|^{1 / p^{\prime}}\left|L_{0}^{\prime}\right|^{1 / q^{\prime}} .
$$

Now, we write (6.278) as follows:

$$
\sum_{K_{1}, L_{0}^{\prime}}\left\langle T \sum_{K_{0}, L_{0}} a_{K_{0}, L_{0}, L_{0}^{\prime}} h_{K_{1} \times L_{0}}, h_{K_{1} \times L_{0}^{\prime}}\right\rangle .
$$

By duality, we obtain the subsequent upper estimate for (6.280):

$$
\sum_{K_{1}, L_{0}^{\prime}}\left\|T \sum_{K_{0}, L_{0}} a_{K_{0}, L_{0}, L_{0}^{\prime}} h_{K_{1} \times L_{0}}\right\|_{H^{p}\left(H^{q}\right)}\left\|h_{K_{1} \times L_{0}^{\prime}}\right\|_{\left(H^{p}\left(H^{q}\right)\right)^{*}} .
$$

Estimate (6.279) and the disjointness of the dyadic intervals (see (J1)) yield

$$
\sum_{K_{1}, L_{0}^{\prime}}\|T\|\left\|\sum_{K_{0}, L_{0}} \max _{L_{0}}\left(\|T\|\left|K_{0}\right|^{1 / p}\left|L_{0}\right|^{1 / q}\left|K_{0}\right|^{1 / p^{\prime}}\left|L_{0}^{\prime}\right|^{1 / q^{\prime}}\right) h_{K_{1} \times L_{0}}\right\|_{H^{p}\left(H^{q}\right)}\left\|h_{K_{1} \times L_{0}^{\prime}}\right\|_{\left(H^{p}\left(H^{q}\right)\right)^{*}} .
$$


Consequently, we obtain

$$
\sum_{K_{1}, L_{0}^{\prime}}\|T\| \sum_{K_{0}} \max _{L_{0}}\left(\|T\|\left|K_{0}\right|^{1 / p}\left|L_{0}\right|^{1 / q}\left|K_{0}\right|^{1 / p^{\prime}}\left|L_{0}^{\prime}\right|^{1 / q^{\prime}}\right)\left\|\sum_{L_{0}} h_{K_{1} \times L_{0}}\right\|_{H^{p}\left(H^{q}\right)}\left\|h_{K_{1} \times L_{0}^{\prime}}\right\|_{\left(H^{p}\left(H^{q}\right)\right)^{*}} .
$$

Thus, 6.283) is bounded from above by

$$
\|T\|^{2} \max _{L_{0}}\left|L_{0}\right|^{1 / q} \sum_{K_{1}, L_{0}^{\prime}} \sum_{K_{0}}\left|K_{0}\right|\left|L_{0}^{\prime}\right|^{2 / q^{\prime}}\left|K_{1}\right| .
$$

Using Hölder's inequality yields

$$
\|T\|^{2} \max _{L_{0}, L_{0}^{\prime}}\left|L_{0}\right|^{1 / q}\left|L_{0}^{\prime}\right|^{2 / q^{\prime}-1}
$$

Inserting $\left|L_{0}\right|,\left|L_{0}^{\prime}\right| \leq \alpha$ (see (4.15)) into (6.284), we obtain the estimate

$$
\|T\|^{2} \alpha^{1 / q^{\prime}}
$$

CAse 18, Group (F7): $K_{0}=K_{0}^{\prime} \neq K_{1}=K_{1}^{\prime}, L_{0}=L_{1} \neq L_{0}^{\prime}=L_{1}^{\prime}(((2)(0))$ (NIL NIL) $((0)(1)))$ - RIGHT VARIANT. In this case, we have to estimate

$$
\sum_{K_{1}, L_{0}, K_{0}, L_{0}^{\prime}}\left\langle T h_{K_{0} \times L_{0}}, h_{K_{0} \times L_{0}^{\prime}}\right\rangle\left\langle T h_{K_{1} \times L_{0}}, h_{K_{1} \times L_{0}^{\prime}}\right\rangle .
$$

We put $a_{K_{0}, L_{0}, L_{0}^{\prime}}=\left\langle T h_{K_{0} \times L_{0}}, h_{K_{0} \times L_{0}^{\prime}}\right\rangle$ and note the estimate

$$
\left|a_{K_{0}, L_{0}, L_{0}^{\prime}}\right| \leq\|T\|\left|K_{0}\right|^{1 / p}\left|L_{0}\right|^{1 / q}\left|K_{0}\right|^{1 / p^{\prime}}\left|L_{0}^{\prime}\right|^{1 / q^{\prime}} .
$$

Now, we write (6.286) as follows:

$$
\sum_{K_{1}, L_{0}}\left\langle T h_{K_{1} \times L_{0}}, \sum_{K_{0}, L_{0}^{\prime}} a_{K_{0}, L_{0}, L_{0}^{\prime}} h_{K_{1} \times L_{0}^{\prime}}\right\rangle .
$$

By duality, we obtain the subsequent upper estimate for (6.288):

$$
\sum_{K_{1}, L_{0}}\left\|T h_{K_{1} \times L_{0}}\right\|_{H^{p}\left(H^{q}\right)}\left\|\sum_{K_{0}, L_{0}^{\prime}} a_{K_{0}, L_{0}, L_{0}^{\prime}} h_{K_{1} \times L_{0}^{\prime}}\right\|_{\left(H^{p}\left(H^{q}\right)\right)^{*}}
$$

Estimate (6.287) and the disjointness of the dyadic intervals (see (J1)) yield

$$
\sum_{K_{1}, L_{0}}\|T\|\left\|h_{K_{1} \times L_{0}}\right\|_{H^{p}\left(H^{q}\right)}\left\|\sum_{K_{0}, L_{0}^{\prime}} \max _{L_{0}^{\prime}}\left(\|T\|\left|K_{0}\right|^{1 / p}\left|L_{0}\right|^{1 / q}\left|K_{0}\right|^{1 / p^{\prime}}\left|L_{0}^{\prime}\right|^{1 / q^{\prime}}\right) h_{K_{1} \times L_{0}^{\prime}}\right\|_{\left(H^{p}\left(H^{q}\right)\right)^{*}} .
$$

Consequently, we obtain

$$
\sum_{K_{1}, L_{0}}\|T\|\left\|h_{K_{1} \times L_{0}}\right\|_{H^{p}\left(H^{q}\right)} \sum_{K_{0}} \max _{L_{0}^{\prime}}\left(\|T\|\left|K_{0}\right|^{1 / p}\left|L_{0}\right|^{1 / q}\left|K_{0}\right|^{1 / p^{\prime}}\left|L_{0}^{\prime}\right|^{1 / q^{\prime}}\right)\left\|\sum_{L_{0}^{\prime}} h_{K_{1} \times L_{0}^{\prime}}\right\|_{\left(H^{p}\left(H^{q}\right)\right)^{*}} .
$$

Thus, (6.291) is bounded from above by

$$
\|T\|^{2} \max _{L_{0}^{\prime}}\left|L_{0}^{\prime}\right|^{1 / q^{\prime}} \sum_{K_{1}, L_{0}} \sum_{K_{0}}\left|K_{0}\right|\left|L_{0}\right|^{2 / q}\left|K_{1}\right| .
$$

Using Hölder's inequality yields

$$
\|T\|^{2} \max _{L_{0}^{\prime}}\left|L_{0}^{\prime}\right|^{1 / q^{\prime}}\left|L_{0}\right|^{2 / q-1} .
$$


Inserting $\left|L_{0}\right|,\left|L_{0}^{\prime}\right| \leq \alpha$ (see (4.15) $)$ into (6.292), we obtain the estimate

$$
\|T\|^{2} \alpha^{1 / q} \text {. }
$$

Summary of Case 17 and Case 18, Combining (6.285) with (6.293) yields

$$
\mathbb{E}_{\theta, \varepsilon} Z^{2} \leq\|T\|^{2} \alpha^{1 / 2}
$$

CASE 19, GRoup (F8): $K_{0}=K_{0}^{\prime} \neq K_{1}=K_{1}^{\prime}, L_{0}=L_{1}^{\prime} \neq L_{0}^{\prime}=L_{1}(((2)(0))((0)$

(1)) (NIL NIL)) - LEFT VARIANT. In this case, we have to estimate

$$
\sum_{K_{1}, L_{0}, K_{0}, L_{0}^{\prime}}\left\langle T h_{K_{0} \times L_{0}}, h_{K_{0} \times L_{0}^{\prime}}\right\rangle\left\langle T h_{K_{1} \times L_{0}^{\prime}}, h_{K_{1} \times L_{0}}\right\rangle .
$$

We put $a_{K_{0}, L_{0}, L_{0}^{\prime}}=\left\langle T h_{K_{0} \times L_{0}}, h_{K_{0} \times L_{0}^{\prime}}\right\rangle$ and note the estimate

$$
\left|a_{K_{0}, L_{0}, L_{0}^{\prime}}\right| \leq\|T\|\left|K_{0}\right|^{1 / p}\left|L_{0}\right|^{1 / q}\left|K_{0}\right|^{1 / p^{\prime}}\left|L_{0}^{\prime}\right|^{1 / q^{\prime}} .
$$

Now, we write (6.295) as follows:

$$
\sum_{K_{1}, L_{0}}\left\langle T \sum_{K_{0}, L_{0}^{\prime}} a_{K_{0}, L_{0}, L_{0}^{\prime}} h_{K_{1} \times L_{0}^{\prime}}, h_{K_{1} \times L_{0}}\right\rangle .
$$

By duality, we obtain the subsequent upper estimate for (6.297):

$$
\sum_{K_{1}, L_{0}}\left\|T \sum_{K_{0}, L_{0}^{\prime}} a_{K_{0}, L_{0}, L_{0}^{\prime}} h_{K_{1} \times L_{0}^{\prime}}\right\|_{H^{p}\left(H^{q}\right)}\left\|h_{K_{1} \times L_{0}}\right\|_{\left(H^{p}\left(H^{q}\right)\right)^{*}}
$$

Estimate (6.296) and the disjointness of the dyadic intervals (see (J1)) yield

$$
\sum_{K_{1}, L_{0}}\|T\|\left\|\sum_{K_{0}, L_{0}^{\prime}} \max _{L_{0}^{\prime}}\left(\|T\|\left|K_{0}\right|^{1 / p}\left|L_{0}\right|^{1 / q}\left|K_{0}\right|^{1 / p^{\prime}}\left|L_{0}^{\prime}\right|^{1 / q^{\prime}}\right) h_{K_{1} \times L_{0}^{\prime}}\right\|_{H^{p}\left(H^{q}\right)}\left\|h_{K_{1} \times L_{0}}\right\|_{\left(H^{p}\left(H^{q}\right)\right)^{*}}
$$

Consequently, we obtain

$$
\sum_{K_{1}, L_{0}}\|T\| \sum_{K_{0}} \max _{L_{0}^{\prime}}\left(\|T\|\left|K_{0}\right|^{1 / p}\left|L_{0}\right|^{1 / q}\left|K_{0}\right|^{1 / p^{\prime}}\left|L_{0}^{\prime}\right|^{1 / q^{\prime}}\right)\left\|\sum_{L_{0}^{\prime}} h_{K_{1} \times L_{0}^{\prime}}\right\|_{H^{p}\left(H^{q}\right)}\left\|h_{K_{1} \times L_{0}}\right\|_{\left(H^{p}\left(H^{q}\right)\right)^{*}}
$$

Thus, (6.300) is bounded from above by

$$
\|T\|^{2} \max _{L_{0}^{\prime}}\left|L_{0}^{\prime}\right|^{1 / q^{\prime}} \sum_{K_{1}, L_{0}} \sum_{K_{0}}\left|K _ { 0 } \left\|\left.L_{0}|| K_{1}\left|\leq\|T\|^{2} \max _{L_{0}^{\prime}}\right| L_{0}^{\prime}\right|^{1 / q^{\prime}} .\right.\right.
$$

Inserting $\left|L_{0}^{\prime}\right| \leq \alpha$ (see (4.15)) into (6.301), we obtain the estimate

$$
\|T\|^{2} \alpha^{1 / q^{\prime}} \text {. }
$$

Case 20, Group (F8): $K_{0}=K_{0}^{\prime} \neq K_{1}=K_{1}^{\prime}, L_{0}=L_{1}^{\prime} \neq L_{0}^{\prime}=L_{1}(((2)(1))$ (NIL NIL) ((0) (0))) - RIGHT VARIANT. In this case, we have to estimate

$$
\sum_{K_{1}, L_{0}^{\prime}, K_{0}, L_{0}}\left\langle T h_{K_{0} \times L_{0}}, h_{K_{0} \times L_{0}^{\prime}}\right\rangle\left\langle T h_{K_{1} \times L_{0}^{\prime}}, h_{K_{1} \times L_{0}}\right\rangle .
$$

We put $a_{K_{0}, L_{0}, L_{0}^{\prime}}=\left\langle T h_{K_{0} \times L_{0}}, h_{K_{0} \times L_{0}^{\prime}}\right\rangle$ and note the estimate

$$
\left|a_{K_{0}, L_{0}, L_{0}^{\prime}}\right| \leq\|T\|\left|K_{0}\right|^{1 / p}\left|L_{0}\right|^{1 / q}\left|K_{0}\right|^{1 / p^{\prime}}\left|L_{0}^{\prime}\right|^{1 / q^{\prime}} .
$$

Now, we write (6.303) as follows:

$$
\sum_{K_{1}, L_{0}^{\prime}}\left\langle T h_{K_{1} \times L_{0}^{\prime}}, \sum_{K_{0}, L_{0}} a_{K_{0}, L_{0}, L_{0}^{\prime}} h_{K_{1} \times L_{0}}\right\rangle .
$$


By duality, we obtain the subsequent upper estimate for (6.305):

$$
\sum_{K_{1}, L_{0}^{\prime}}\left\|T h_{K_{1} \times L_{0}^{\prime}}\right\|_{H^{p}\left(H^{q}\right)}\left\|\sum_{K_{0}, L_{0}} a_{K_{0}, L_{0}, L_{0}^{\prime}} h_{K_{1} \times L_{0}}\right\|_{\left(H^{p}\left(H^{q}\right)\right)^{*}} .
$$

Estimate (6.304) and the disjointness of the dyadic intervals (see (JJ1) yield

$$
\sum_{K_{1}, L_{0}^{\prime}}\|T\|\left\|h_{K_{1} \times L_{0}^{\prime}}\right\|_{H^{p}\left(H^{q}\right)}\left\|\sum_{K_{0}, L_{0}} \max _{L_{0}}\left(\|T\|\left|K_{0}\right|^{1 / p}\left|L_{0}\right|^{1 / q}\left|K_{0}\right|^{1 / p^{\prime}}\left|L_{0}^{\prime}\right|^{1 / q^{\prime}}\right) h_{K_{1} \times L_{0}}\right\|_{\left(H^{p}\left(H^{q}\right)\right)^{*}} .
$$

Consequently, we obtain

$$
\sum_{K_{1}, L_{0}^{\prime}}\|T\|\left\|h_{K_{1} \times L_{0}^{\prime}}\right\|_{H^{p}\left(H^{q}\right)} \sum_{K_{0}} \max _{L_{0}}\left(\|T\|\left|K_{0}\right|^{1 / p}\left|L_{0}\right|^{1 / q}\left|K_{0}\right|^{1 / p^{\prime}}\left|L_{0}^{\prime}\right|^{1 / q^{\prime}}\right)\left\|\sum_{L_{0}} h_{K_{1} \times L_{0}}\right\|_{\left(H^{p}\left(H^{q}\right)\right)^{*}} .
$$

Thus, 6.308 is bounded from above by

$$
\|T\|^{2} \max _{L_{0}}\left|L_{0}\right|^{1 / q} \sum_{K_{1}, L_{0}^{\prime}} \sum_{K_{0}}\left|K_{1}\right|\left|K_{0}\right|\left|L_{0}^{\prime}\right| \leq\|T\|^{2} \max _{L_{0}}\left|L_{0}\right|^{1 / q} .
$$

Inserting $\left|L_{0}\right| \leq \alpha$ (see (4.15)) into (6.309), we obtain the estimate

$$
\|T\|^{2} \alpha^{1 / q} \text {. }
$$

Summary of Case 19 and Case 20, Combining (6.302) with (6.310) yields

$$
\mathbb{E}_{\theta, \varepsilon} Z^{2} \leq\|T\|^{2} \alpha^{1 / 2} .
$$

Summary for $Z$. Combining (6.151), 66.159), (6.167), 66.192), 6.209), 66.226), (6.175), (6.243), 6.260), 6.277), (6.294) and (6.311) yields

$$
\mathbb{E}_{\theta, \varepsilon} Z^{2} \leq 12\|T\|^{2} \alpha^{1 / 2}
$$

\section{Acknowledgments.}

It is my pleasure to thank P.F.X. Müller for many helpful discussions. Supported by the Austrian Science Foundation (FWF) Pr.Nr. P28352.

\section{REFERENCES}

[1] G. Blower. The Banach space $B\left(l^{2}\right)$ is primary. Bull. London Math. Soc., 22(2):176-182, 1990.

[2] J. Bourgain. On the primarity of $H^{\infty}$-spaces. Israel J. Math., 45(4):329-336, 1983.

[3] J. Bourgain and L. Tzafriri. Invertibility of "large" submatrices with applications to the geometry of Banach spaces and harmonic analysis. Israel J. Math., 57(2):137-224, 1987.

[4] P. W. Jones. BMO and the Banach space approximation problem. Amer. J. Math., 107(4):853-893, 1985.

[5] N. J. Laustsen, R. Lechner, and P. F. X. Müller. Factorization of the identity through operators with large diagonal. ArXiv e-prints, Sept. 2015.

[6] R. Lechner. Factorization in mixed norm Hardy and BMO spaces. Studia Math., to appear. Preprint available on ArXiv.

[7] R. Lechner. Direct sums of finite dimensional $S L_{n}^{\infty}$ spaces. ArXiv e-prints, Sept. 2017.

[8] R. Lechner. Dimension dependence of factorization problems: Hardy spaces and $S L \_n^{\infty}$. ArXiv e-prints, Feb. 2018

[9] R. Lechner and P. F. X. Müller. Localization and projections on bi-parameter BMO. Q. J. Math., 66(4):1069-1101, 2015.

[10] P. F. X. Müller. On projections in $H^{1}$ and BMO. Studia Math., 89(2):145-158, 1988. 
DIMENSION DEPENDENCE OF FACTORIZATION PROBLEMS: BI-PARAMETER HARDY SPACE\$

[11] P. F. X. Müller. Isomorphisms between $H^{1}$ spaces, volume 66 of Instytut Matematyczny Polskiej Akademii Nauk. Monografie Matematyczne (New Series) [Mathematics Institute of the Polish Academy of Sciences. Mathematical Monographs (New Series)]. Birkhäuser Verlag, Basel, 2005.

[12] P. F. X. Müller. Two remarks on primary spaces. Math. Proc. Cambridge Philos. Soc., 153(3):505-523, 2012.

[13] H. M. Wark. A class of primary Banach spaces. J. Math. Anal. Appl., 326(2):1427-1436, 2007.

[14] H. M. Wark. The $l^{\infty}$ direct sum of $L^{p}(1<p<\infty)$ is primary. J. Lond. Math. Soc. (2), 75(1):176-186, 2007.

Richard Lechner, Institute of Analysis, Johannes Kepler University Linz, Altenberger Strasse 69, A-4040 Linz, Austria

E-mail address: richard.lechner@jku.at 